Florida International University

FIU Digital Commons

FIU Electronic Theses and Dissertations

University Graduate School

3-28-2005

\title{
The use of capillary electrophoresis (CE) in drug analysis
}

Agnes D. Garcia

Follow this and additional works at: https://digitalcommons.fiu.edu/etd

Part of the Other Life Sciences Commons

\section{Recommended Citation}

Garcia, Agnes D., "The use of capillary electrophoresis (CE) in drug analysis" (2005). FIU Electronic Theses and Dissertations. 3928.

https://digitalcommons.fiu.edu/etd/3928

This work is brought to you for free and open access by the University Graduate School at FIU Digital Commons. It has been accepted for inclusion in FIU Electronic Theses and Dissertations by an authorized administrator of FIU Digital Commons. For more information, please contact dcc@fiu.edu. 


\section{FLORIDA INTERNATIONAL UNIVERSITY}

Miami, Florida

THE USE OF CAPILLARY ELECTROPHORESIS (CE) IN DRUG ANALYSIS

A thesis submitted in partial fulfillment of the

requirements for the degree of

MASTER OF SCIENCE

in

FORENSIC SCIENCE

by

Agnes D. Garcia

2005 
To: Dean R. Bruce Dunlap

College of Arts and Sciences

This thesis, written by Agnes D. Garcia, and entitled The Use of Capillary

Electrophoresis (CE) in Drug Analysis, having been approved in respect to style and intellectual content, is referred to you for judgment.

We have read this thesis and recommend that it be approved.

Kenneth Furton

Bruce McCord

Jose R. Almirall, Major Professor

Date of Defense: March 28, 2005

The thesis of Agnes D. Garcia is approved.

Dean R. Bruce Dunlap College of Arts and Sciences

Dean Douglas Wartzok University Graduate School

Florida International University, 2005 
(C) Copyright 2005 by Agnes D. Garcia

All rights reserved. 


\section{DEDICATION}

I dedicate this thesis to my mother Miriam Delgado and Jennifer Winokur. Without their patience, understanding, support, and most of all love, the completion of this work would not have been possible. 


\section{ACKNOWLEDGMENTS}

The author would like to take this opportunity to thank several people who played key roles in this research project and were instrumental in providing an excellent educational experience. First, I would like to thank my advisor, Dr. Jose Almirall, who supported me throughout this endeavor. Without his support and patience, I would not have been able to complete this project or the degree of Master of Science at FIU. I would also like to thank him for the valuable experience that he has given me through the years. I would also like to thank the other two members of my committee, Dr. Ken Furton and Dr. Bruce McCord, for their time and support in this thesis project.

I would also like to express great gratitude to Senior Forensic Chemist Ira Lurie, at the DEA Special Testing and Research Laboratory, for valuable information on the techniques of capillary electrophoresis and high performance liquid chromatography. Gratitude is also extended to Allen Catterton at the DEA Southeast Laboratory in Miami, Florida, for sharing his expertise and experience in GHB analysis. 


\section{ABSTRACT OF THE THESIS \\ THE USE OF CAPILLARY ELECTROPHORESIS (CE) IN DRUG ANALYSIS \\ by \\ Agnes D. Garcia}

Florida International University, 2005

Miami, Florida

Professor Jose R. Almirall, Major Professor

Capillary electrophoresis is currently a very powerful technique for the analysis of seized drugs. A rapid analytical CE method for the screening and quantification of GHB and GBL was achieved using 300mM CTAC/25mM phosphate buffer $\mathrm{pH} 6.3$. Reversed phase HPLC was achieved using $25 \mathrm{mM}$ phosphate buffer $\mathrm{pH} 6.5$ and a $\mathrm{C} 18$ Aqua column. Chiral separation of 9 amphetamine type stimulants was obtained using a highly sulfated gamma-cyclodextrin as a chiral selector. MECC and CZE were compared for the analysis of psilocybin, while a rapid and robust method is presented for the analysis of major opium alkaloids, using dynamically coated capillary columns. The column is coated with a polycation followed by a polyanion coating, using a commercial reagent kit. Using a background electrolyte $\mathrm{pH}$ of 2.5 with the addition of hydroxypropyl-betacyclodextrin and dimethyl-beta-cyclodextrin, the analysis of morphine, papaverine, codeine, noscapine, and thebaine in opium samples was obtained with great resolution. Finally, separation of common benzodiazedpines was also investigated using CZE and a pH 2.5 phosphate buffer. 


\section{TABLE OF CONTENTS}

CHAPTER

PAGE

I. INTRODUCTION

Capillary Electrophoresis

Capillary Zone Electrophoresis

Micellar Electrokinetic Capillary Chromatography

Cyclodextrins

Dynamic Coating

Liquid Chromatography

Reverse Phase Chromatography

Forensic Applications

Purpose of Project

$\begin{array}{ll}\text { II. DRUGS OF INTEREST } & 27\end{array}$

$\begin{array}{ll}\text { Gamma-hydroxybutyric Acid } & 27\end{array}$

$\begin{array}{ll}\text { Phenylethylamines } & 36\end{array}$

$\begin{array}{lr}\text { Psilocybin } & 40\end{array}$

Opium $\quad 42$

Benzodiazepines $\quad 45$

$\begin{array}{ll}\text { III. CE METHODOLOGY } & 48\end{array}$

GHB Analysis $\quad 48$

Phenylethylamines Analysis $\quad 50$

Psilocybin Analysis $\quad 51$

Opium $\quad 52$

Benzodiazepines $\quad 53$

IV. HPLC METHODOLOGY 54

GHB Analysis $\quad 54$

Phenylethylamines Analysis $\quad 55$

Psilocybin Analysis $\quad 55$

Opium Analysis $\quad 56$

V. CE RESULTS AND DISCUSSION 57

$\begin{array}{ll}\text { GHB Analysis } & 57\end{array}$

$\begin{array}{ll}\text { Phenylethylamines Analysis } & 67\end{array}$

Psilocybin Analysis $\quad 83$

Opium Analysis $\quad 89$

Benzodiazepines Analysis $\quad 91$

VI. HPLC RESULTS AND DISCUSSION 98

GHB Analysis $\quad 98$

$\begin{array}{ll}\text { Phenylethylamines Analysis } & 103\end{array}$ 
Psilocybin Analysis

105

Opium Analysis

Benzodiazepines Analysis

VII. CONCLUSIONS

113

LIST OF REFERENCES 
61. Linearity of flunitrazepam $(0.10$ to $1.1 \mathrm{mg} / \mathrm{ml})$, using $50 \mathrm{mM}$ phosphate $\mathrm{pH} 2.5,210 \mathrm{~nm}$, and a 50um x $48.5 \mathrm{~cm}$ column on a Hewlett Packard $3 \mathrm{D} \mathrm{CE}$

62. Common benzodiazepines separated using the CZE method of $50 \mathrm{mM}$ phosphate $\mathrm{pH} 2.5,210 \mathrm{~nm}$, and a $50 \mathrm{um} \times 48.5 \mathrm{~cm}$ column on a Hewlett Packard 3D CE

63. GHB (3.3 minutes) eluted using a $25 \mathrm{mM}$ phosphate $\mathrm{pH} 6.5,195 \mathrm{~nm}$, and $250 \times 4.6 \mathrm{~mm} \mathrm{C18}$ Aqua column on a Hewlett Packard 3D CE

64. GHB and GBL using $25 \mathrm{mM}$ phosphate $\mathrm{pH} 6.5 / 10 \%$ methanol, $195 \mathrm{~nm}$, and a $250 \times 4.6 \mathrm{~mm}$ C18 Aqua column on an Agilent HPLC 1100

65. GHB and GBL using $25 \mathrm{mM}$ phosphate $\mathrm{pH} 6.5,195 \mathrm{~nm}$, and a $150 \mathrm{x}$ 4.6mm C18 Aqua column on an Agilent HPLC 1100

66. Psilocybin standard using an acetonitrile/methanol buffer system on an Agilent HPLC 1100

67. Mushroom 4 using an acetonitrile/methanol buffer system on an 106 Agilent HPLC 1100

68. Opium analysis via $\mathrm{CE}$ method 108

69. Opium analysis via HPLC method 109

70. Flunitrazepam standard $(1.0 \mathrm{mg} / \mathrm{mL})$ using a 5 um ODS $150 \times 3.2 \mathrm{~mm}$ 110 column, $210 \mathrm{~nm}$, and a $50 / 50$ phosphate $\mathrm{pH} 2.3$ /acetonitrile buffer system on an Agilent HPLC 1100

71. Diazepam standard $(1.0 \mathrm{mg} / \mathrm{mL})$ using a $5 \mathrm{um}$ ODS $150 \times 3.2 \mathrm{~mm}$ column, $210 \mathrm{~nm}$, and a 50/50 phosphate $\mathrm{pH} 2.3$ /acetonitrile buffer system on an Agilent HPLC 1100

72. UV spectra of flunitrazepam

73. UV spectra of diazepam 


\section{INTRODUCTION}

"The goal of the forensic scientist is to resolve...complex samples into identifiable constituents, free from the interference of other substances. The methods used to achieve this goal must be reliable, rapid, economical, and provide information that is unequivocal. It is also important to preserve as much of the evidence as possible. Samples are often very limited; therefore analytical techniques need to be nondestructive or use limited amounts of sample. CE is an analytical tool that shows great promise in addressing these requirements." Northrop et. al., J. Cap. Electrophoresis 1994; 001(2):158-168. ${ }^{1}$

\subsection{Capillary Electrophoresis}

Capillary Electrophoresis (CE) is now a well-established separation technique utilized throughout the different disciplines in the Forensic Sciences. It is currently utilized in the analysis of explosives, toxicology, DNA testing, and drug chemistry. The different modes of $\mathrm{CE}$ allow for the analysis of a large variety of abused drugs. While free zone capillary electrophoresis (CZE) is a good separation technique for basic and acidic drugs, micellar electrokinetic capillary chromatography (MECC) allows for the analysis of not only the basic and acidic compounds but also neutrals. Chirality determination is also possible with the use of cyclodextrin systems.

$\mathrm{CE}$ is a technique for the separation of substances, based on the different migration rates of charged particles in an electric field. Like High Performance Liquid Chromatography (HPLC), it is applicable to the analysis of nonvolatile, polar, and thermally degradable compounds. However, CE is a more efficient technique than HPLC. The mass limit of detection is 100 times lower than HPLC, and its separation efficiency is in the hundreds of thousands of theoretical plates as opposed to thousands with HPLC. ${ }^{2}$ 
The instrument setup of a CE contains a high voltage power supply, two buffer reservoirs, two electrodes, a capillary column, a detector, and a replenishment system (figure 1).

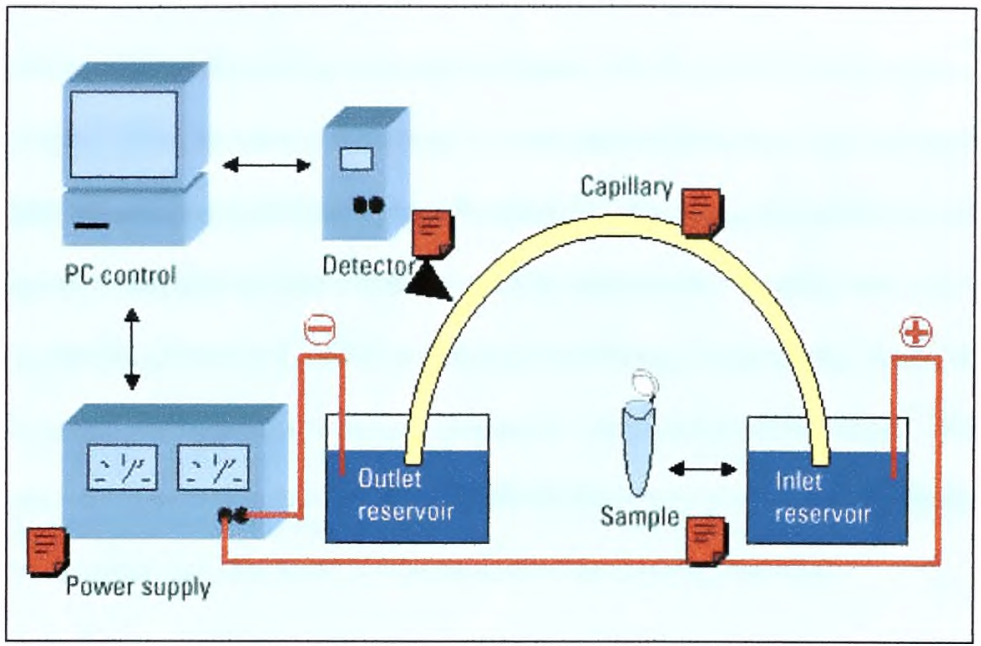

* Picture obtained from DEA Senior Forensic Chemist Ira Lurie.

Figure 1: Scheme of a capillary electropherograph.

CE systems, in general, use a UV-VIS detector and fused silica columns. These columns are UV transparent and are coated with polyamide, making them very durable. ${ }^{3}$ The internal diameter of most silica columns range between 25 and $75 \mathrm{um}$, while their length is usually between $30-100 \mathrm{~cm}^{3}$ Silica columns are also of low cost making them very desirable in laboratories. Capillaries are usually purchased in bulk, at a few dollars per meter. These columns have a high electrical resistance, enabeling them to withstand very high electrical fields, such as 100-500 volts per centimeter with low generation of 
heat. ${ }^{3}$ Low current is desirable because it reduces joule heating and prevents the broadening of peaks. Increased heating “....can cause nonuniform temperature gradients, local changes in viscosity, and subsequent zone broadening." 3

Specialized CE columns are also available. The proprietary design of a high sensitivity cell has allowed an extended detection path length of $1.2 \mathrm{~mm}$, while reducing stray light. ${ }^{3}$ Thus, increasing sensitivity by more than 10 -fold over standard capillaries. Bubble cell columns (extended light path columns) also extend the detection path length. However, while they are less expensive than the high sensitivity cells, they only increase sensitivity by a factor of $5 .^{3} \mathrm{CEC}$ columns are increasing in popularity. Capillaries of 50 $\mathrm{cm}$ in length can be packed with $3 \mu \mathrm{m}$ porous or $1.5 \mu \mathrm{m}$ pellicular packings. ${ }^{3}$ These columns can generate hundreds of thousands of theoretical plates. ${ }^{3}$ Furthermore, the current remains low due to the low resistance of the packing material.

\subsection{Capillary Zone Electrophoresis}

CZE is a fast and easy separation technique applicable to most substances. It utilizes fused silica columns, with the ends of the capillary column immersed in two buffer reservoirs with electrodes. High voltage is then applied to the column, usually 20 to $30 \mathrm{kV}$. Separation is based on the different electrophoretic mobilities of the solutes. In turn, the mobilities of the analytes towards the cathode are a function of charge and size. The degree of ionization that occurs on the column, which is dependent on the $\mathrm{pH}$ of the running buffer, affects the charge of the solute. ${ }^{3}$ 
The inner surface of the capillary column contains silanol groups, which at a $\mathrm{pH}$ of 2-9 ionize creating an excess of negative charge. The $\mathrm{pH}$ of the buffer used determines the quantity of ionized silanol groups (SiO-), which in turn attract the positive ions from the background electrolyte or buffer. ${ }^{3}$ This ion attraction forms an electrical double layer, called the stern layer, which is closest to the capillary wall and static. ${ }^{3}$ The outer layer or Outer Helmholtz Plane (OHP) is more diffuse and when voltage is applied, the cations migrate toward the cathode carrying with them associated waters of hydration. ${ }^{3}$ It is the cohesive nature of the H-bonding of waters of hydration to the water molecules, that pulls the entire background electrolyte solution towards the cathode. This pull is the bulk electroosmotic flow (EOF) of the buffer solution moving towards the negatively charged electrode, propelling cationic, anionic, and neutral species toward the cathode (detector). ${ }^{3}$

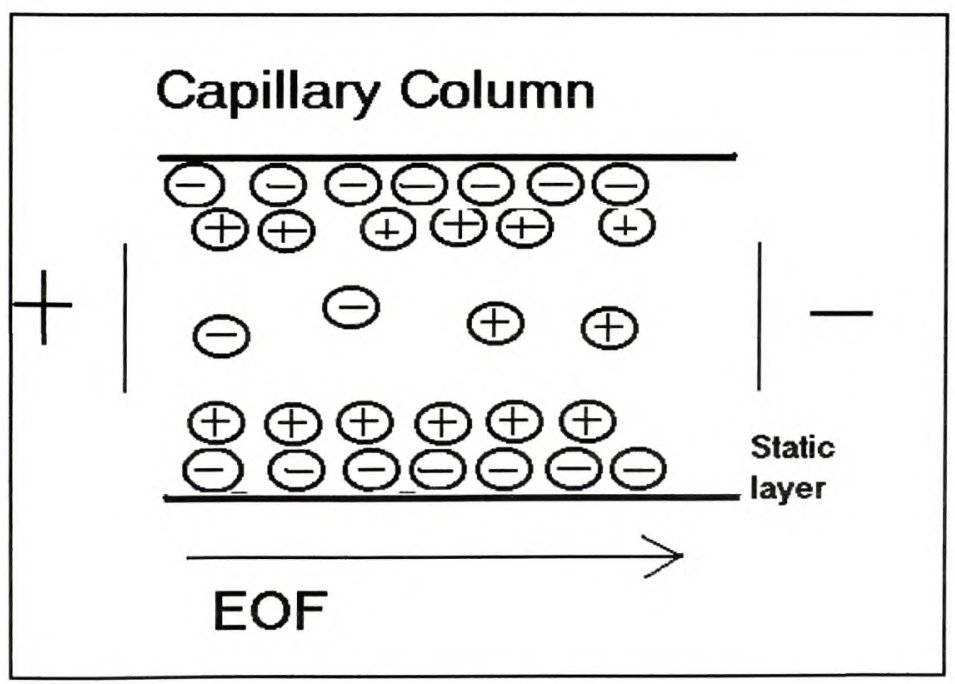

Figure 2: A schematic diagram of electroosmotic flow. 
The anionic and neutral species are carried by the velocity of the EOF, towards the cathode. Therefore, the order of migration will be as follows, cations, neutrals, and anions (figure 2). Since the neutral compounds don't have a charge of their own, they migrate as one peak, without species distinction. CZE is a valuable mode for the separation of basic and acidic compounds, but unsuitable for the separation of neutral substances.

\subsection{Micellar Electrokinetic Capillary Chromatography (MECC)}

Since CZE is not applicable to the analysis of uncharged compounds, the CZE method can be modified by adding a surfactant. ${ }^{4} \mathrm{MECC}$, a technique first applied to drug analysis by Weinberger and Lurie, involves the introduction of micelles to the buffer solution. ${ }^{4}$ These authors reported the use of the surfactant sodium dodecyl sulfate (SDS) or the salt cetyltrimethylammonium chloride (CTAC) to assist in the separation. These surfactants were added at concentrations level above which micelles form. "Micelles are spherical in shape with its surface comprised of the polar groups of the surfactant molecule, while its core region is comprised of the hydrophobic region (alkyl groups) of the surfactant." "This concentration of surfactants is known as the critical micelle concentration." ${ }^{2}$ Micelles can absorb nonpolar compounds into their hydrocarbon interior. The nonpolar species are then said to be solubilized. ${ }^{6}$ The most commonly used micelle is SDS. It is an anionic micelle with a large negative charge surface. This negative charge gives SDS micelles a high electrophoretic mobility toward the positive electrode, slowing the rate in which they migrate towards the cathode. The micelles are still carried towards the cathode due to the presence of EOF. Analytes partition between 
the aqueous phase and the hydrocarbon phase in the interior of the micelles. This equilibrium is dependent on the polarity of each sample component. Polar compounds tend to spend more time in the aqueous phase, while nonpolar solutes spend more time in the hydrocarbon environment (figure 3). Thus, separations are based on differences in the distribution coefficients. The distribution coefficient $(\mathrm{K})$ is defined as follows ${ }^{5}$ :

$$
\mathrm{K}=\mathrm{k} /\left(\mathrm{C}_{\mathrm{sf}}-\mathrm{CMC}\right) \mathrm{v}
$$

where $\mathrm{k}$ is the capacity factor, $\mathrm{C}_{\mathrm{sf}}$ is the surfactant concentration, $v$ is the partial specific volume of the micelle, and $\mathrm{CMC}$ is the critical micelle concentration.

\section{Capillary Column}

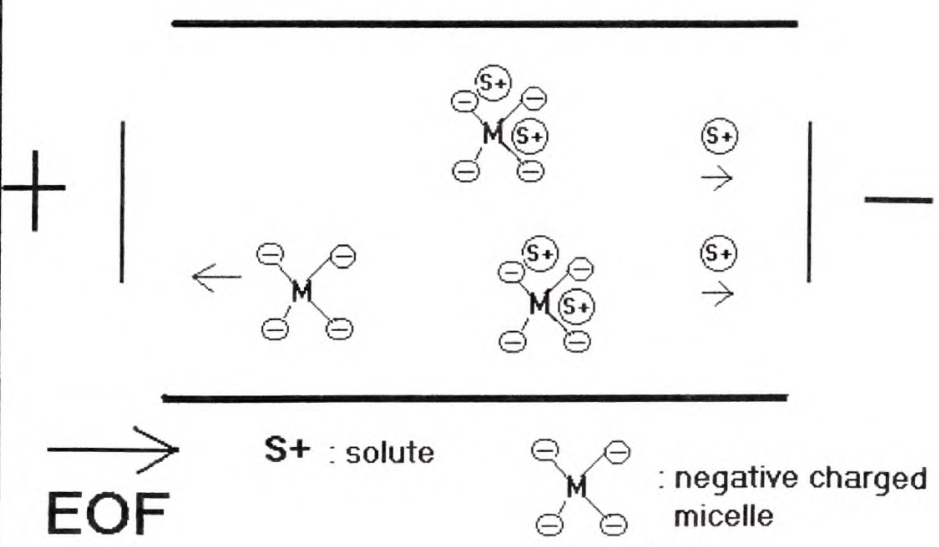

Figure 3: Illustration of negatively charged micelle separation scheme. 


\subsection{Cyclodextrin systems}

In order to achieve chiral analysis by $\mathrm{CE}$, a chiral selector must be added to the buffer, such as chiral crown ethers, chiral surfactants, or cyclodextrins. However, cyclodextrins (CDs) are the most widely used. The addition of cyclodextrins to the running buffer has proven to be a very useful approach for the separation of enantiomers. Cyclodextrins are carbohydrate-derived materials obtained by starch fermentation, that form inclusion compounds with many organic and inorganic compounds. ${ }^{5}$ The cyclodextrins form a family of 3 principal polysaccharide oligomers, designated as alpha, beta, and gamma. The alpha $\mathrm{CD}$ has 6 glucopyranose units attached to its structure. ${ }^{5}$ The beta has 7 and the gamma has 8 (figure 4). Other important properties of CDs include their molecular weights, solubility, cavity diameter, and pKa (which ranges from 12.33 for the $\alpha-C D, 12.20$ for the $\beta-C D$, and 12.08 for the $\gamma-C D){ }^{5}$ Cyclodextrins are useful additives due to their low UV absorptivity. For drug chemistry, this is very desirable since most controlled substances absorb in the UV range. Furthermore, cyclodextrins have an excellent chemical stability over the wide $\mathrm{pH}$ range of $2-10 .^{5}$ 


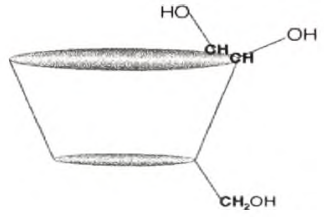

Parent Cyclodextrins $\alpha$

Glucose Units

Molecular Weight

Water solubility (gms/100mL)

Cavity Diameter $(\AA)$

Cavity Volume $(\AA)^{3}$
6

973

12.7

4.7-5.3

$\sim 174$

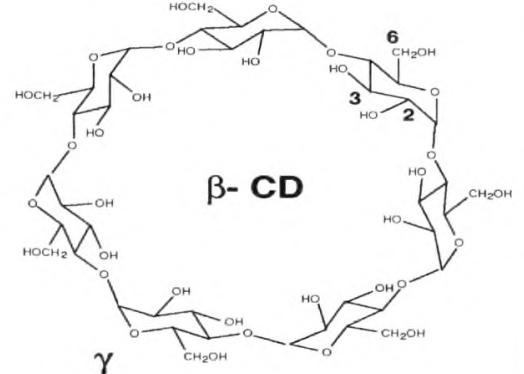

8

1297

$\begin{array}{ll}1.88 & 25.6 \\ 6.0-6.5 & 7.5-8.3\end{array}$

$\sim 262 \sim 472$

* Figure obtained from DEA Senior Forensic Chemist Ira Lurie.

Figure 4: Important characteristics of parent cyclodextrins.?

The inner surface of a CD cavity is hydrophobic and the outer surface is hydrophilic, while the circumference contains chiral secondary hydroxyl groups. ${ }^{7}$

Selective inclusion complexes form when the hydrophobic portion of the solute interacts with the CD cavity and forms H-bonds with the external hydroxyl groups. ${ }^{7}$ Thus, chiral determination occurs due to the difference in stability or mobility of the inclusion complexes formed by the 2 isomers (figure 5). 


\section{Capillary Column}

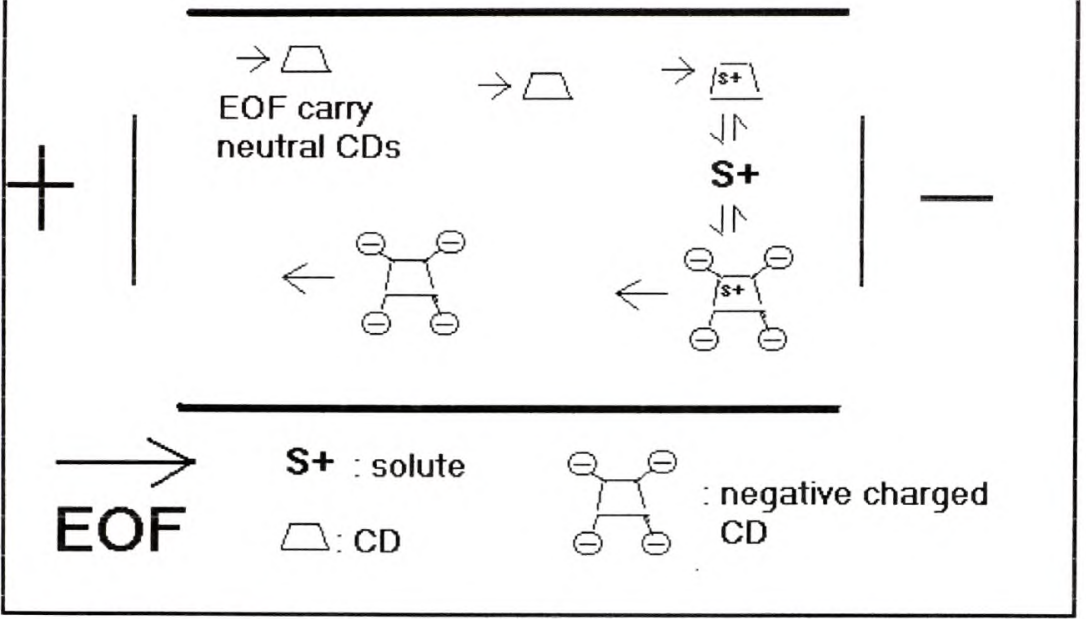

Figure 5: Illustration of negatively charged cyclodextrin separation scheme.

Cyclodextrins are soluble in water and their solubility varies according to their type and structure. This is a desirable characteristic since most electrolytes utilized in CE methods are aqueous solutions. Solubility varies with the size of the molecule. Beta CDs, the least water soluble, due to its rigid CD network of hydrogen bonds between its secondary hydroxyl groups (figure 4). ${ }^{5}$ Gamma has the highest solubility and also the highest cavity, which means that analytes with substituted phenyl rings can form inclusion complexes. ${ }^{5}$ It is important to match the size of the guest molecule to the individual cyclodextrin. Therefore, the alpha $\mathrm{CD}$ is often used for unsubstituted aromatic rings. However, it is not essential for the guest molecule to fit completely into the 
molecular cavity, assuming that the side chain groups are accommodated. ${ }^{5}$ Optical isomers form the inclusion complex at different rates, which allows for a difference in electrophoretic mobility and thus, a separation.

Neutral CDs are frequently derivatized to create charged CDs. Anionic CD derivatives commonly comprise of carboxyalkylated, sulfoalkylated,sulfated, and phosphorylated CDs, while amino and ammonium derivatives are used for cationic CD derivatives. ${ }^{5}$ Charged CDs have self-electrophoretic mobility, as oppose to neutral CDs, which make them more desirable for enantiomeric analysis. CD derivatives can also have higher electrostatic interactions with analytes, than neutral CDs. ${ }^{5}$

\subsection{Dynamic Coating}

Chevigne and Janssens developed the procedure known as Dynamic Coating, which entails the coating the capillary column first with a polycation then a polyanion. ${ }^{8}$ While the first polycation coating coats the column with an excess of negative charge, the second polyanion coating forms a new, altered capillary wall. ${ }^{9}$ This capillary wall now contains a new layer of an excess of negatively charged sites. It is this secondary, modified capillary wall that give rise to a very reproducible, stable, and enhanced EOF. ${ }^{8}$ Graul and Schlenoff also achieved a more stable and reproducible EOF, by adding a polymeric additive to their background electrolyte. ${ }^{10}$ This technique also permits the development of EOF with low $\mathrm{pH}$ electrolyte backgrounds ( $\mathrm{pH}$ from 2.5), permitting the rapid analysis of basic drugs. ${ }^{9}$ Furthermore, analyte diffusion is reduced. ${ }^{9}$ High concentration buffers $(50-100 \mathrm{mM})$ can also be used with this technique providing superior sample stacking, which improves peak shape and sensitivity of detection. ${ }^{9}$ 
Ordinary, highly concentrated buffers reduce EOF by reducing the electrical double layer This doesn't occur with dynamic coating because the electrical double layer is consistently being created before every injection through the coating of the capillary column. The electrodispersion associated with low buffer concentration is also eliminated. ${ }^{9}$ In general the use of dynamic coating improves analysis speed at low $\mathrm{pH}$ and provides a more stable EOF, giving better migration time reproducibility.

\subsection{Liquid Chromatography}

Liquid chromatography is divided into two main types: column chromatography and planar chromatography. ${ }^{11}$ Planar chromatography involves thin layer chromatography and paper chromatography, while column chromatography consists of the different modes of high performance liquid chromatography (HPLC). ${ }^{11}$ HPLC is a chromatographic separation technique involving a liquid mobile phase and a solid stationary phase. It allows for the analysis of compounds that can be challenging by gas chromatography, such as thermally degradable, nonvolatile, or polar compounds, such as GHB and the phenylethylamines. Since HPLC is a nondestructive technique, it can be utilized as a preparative technique. Quantities of purified compound in a mobile phase can be collected through fraction collection.

Like CE, HPLC predominantly uses a UV/VIS detector, which is sensitive and selective. Only compounds which absorb UV radiation can provide a response. However, many controlled substances have no chromophores, with marginal absorptivity, and require detection at low UV values $(185 \mathrm{~nm}-210 \mathrm{~nm}))^{11}$ There are a number of other 
detectors available for drug analysis, including refractive index and fluoresces. However, these are either too selective or have limited sensitivity. HPLC uses a solvent system (figure 6), which plays an important role in the optimization of any separation method. For example, methanol may be preferred over acetonitrile for the separation of ionic samples, which are more hydrophobic samples. Most buffers have a greater solubility in methanol-water mixtures than in mobile phases containing acetonitrile or tetrahydrofuran. ${ }^{11}$

\section{Flow delivered by pump} can be 0.5 to $15 \mathrm{~mL}$ a minute

$1-10 \mathrm{~mL}$ of sample can be injected.

UVNIS

Detector most commonly used.
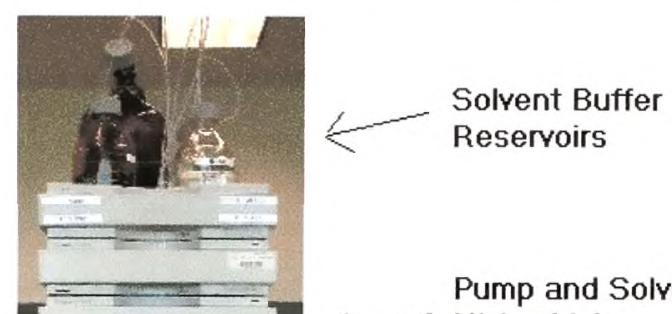

Pump and Solvent Mixing Valye

Sample Injector

Column

Compartment

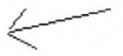

Detector

Figure 6: A depiction of an HPLC instrument. 


\subsection{Reverse Phase}

For drug analysis, the most common mode of HPLC used is reverse phase chromatography. It involves the partitioning between a polar mobile phase and a nonpolar stationary phase. The stationary phase consists of a chain of hydrocarbons (C8 or C18 column) which is less polar than the mobile phase. ${ }^{\text {" }}$ As water or buffer content in the mobile phase increases, the more polar the mobile phase becomes. In contrast, adding an organic solvent to the mobile phase, decreases the mobile phase's polarity. The stationary phase $(\mathrm{C} 8$ or $\mathrm{C} 18)$ retains the less polar or less water soluble compounds. $^{11}$

The temperature of the column compartment can also be an important factor for optimizing a separation method. Although temperature generally has little effect for reverse phase separations of neutral compounds, it can have significant effects for ionic samples. ${ }^{12}$ Temperature can affect the ionization of the sample compounds, the hydrophobic retention of the ionized molecules versus the non-ionized molecules, and the silanol interactions with the ionized species. ${ }^{12}$ Temperature will have the most effect in selectivity, when $\mathrm{pH}$ values are used that result in the partial ionization of the species of interest. $^{12}$ However, high temperatures in combination with either low or very high $\mathrm{pH}$ can lead to a loss of bonded phase of most reverse phase columns. ${ }^{12}$ Resolution in HPLC separations is a result of the degree of retention of a compound. The larger the retention factor $(\mathrm{k})$, the better the resolution. In general, for every degree that temperature is increased, the retention factor will be decreased by $1-2 \% .^{12}$ The resolution obtained with HPLC columns is dependent on the properties of the column and the stationary phase. ${ }^{12}$ 
Longer and narrower columns increase efficiency. Particle size is also important as smaller particles increase the efficiency of the column.

Ion-pair chromatography is an ideal technique for the analysis of drug samples. With this technique, a counter ion can form a lipophilic complex with the salt of a drug is added to the mobile phase. ${ }^{13}$ In the analysis of controlled substances, alkysulfonates can be used as counter ions at a low $\mathrm{pH}^{13}$ Weak acids can be analyzed through ion suppression. The analysis of basic, acidic, and neutral compounds can be performed simultaneously by neutralizing the acid and forming ion pairs with the basic species. ${ }^{13}$ In 1980, Ira Lurie described the use of methanesulfonic acid as a counter ion for the analysis of cocaine, LSD, and PCP. ${ }^{13}$ A surfactant such as SDS can also be added to the mobile phase ("soap chromatography"), for the analysis of complex matrices like PCP on mint leaves or parsley. ${ }^{13}$ Ion pairing allows the compound of interest to be moved to a region past the elution of sample interferences, by increasing the capacity factor ( $\left.k^{\prime}\right)$ and retention of the compound. ${ }^{11}$

HPLC separation of enantiomers requires the creation of a chiral environment. The compound of interest can be reacted with a chiral compound or can be made to interact with a chiral phase, in order to form 2 diastereomers. The 2 diastereomers will then have different chemical and physical properties allowing for their separation. However, this option is time consuming and has the possibility of causing racemization. A chiral mobile phase can also be utilized, by adding a chiral mobile phase additive (CMPA) to the buffer. ${ }^{11}$ CMPAs are usually expensive and have detection difficulties, resulting in poorly shaped peaks with low plate numbers. ${ }^{\text {" }}$ A chiral stationary phase can 
also be used, such as a beta-cyclodextrin column. In these phases, a chiral substance is chemically bonded to a stationary phase, which can interact with the analyte enantiomers, forming transient complexes. The differences in retention times for the two enantiomers is the result of the differences in binding strength of the complexes.

\subsection{Forensic Drug Applications}

\section{Capillary Electrophoresis}

The application of micelles in the analysis of seized drugs was demonstrated by Weinberger and Lurie in $1991 .{ }^{4}$ Using a system of $85 \mathrm{mM}$ SDS $/ 8.5 \mathrm{mM}$ Borate/ $8.5 \mathrm{mM}$ Phosphate $/ 15 \%$ Acetonitrile at a $\mathrm{pH}$ of 8.5 , they separated 18 common controlled substances (figure 7). ${ }^{4}$ This separation clearly shows how MECC permits for the separation of neutral and basic solutes, including thermally degradable compounds like psilocybin, and non volatile compounds like amphetamine hydrochloride. Weinberger and Lurie later extended this method to include the separation of 34 illicit drugs and drug impurities. $^{4}$ 


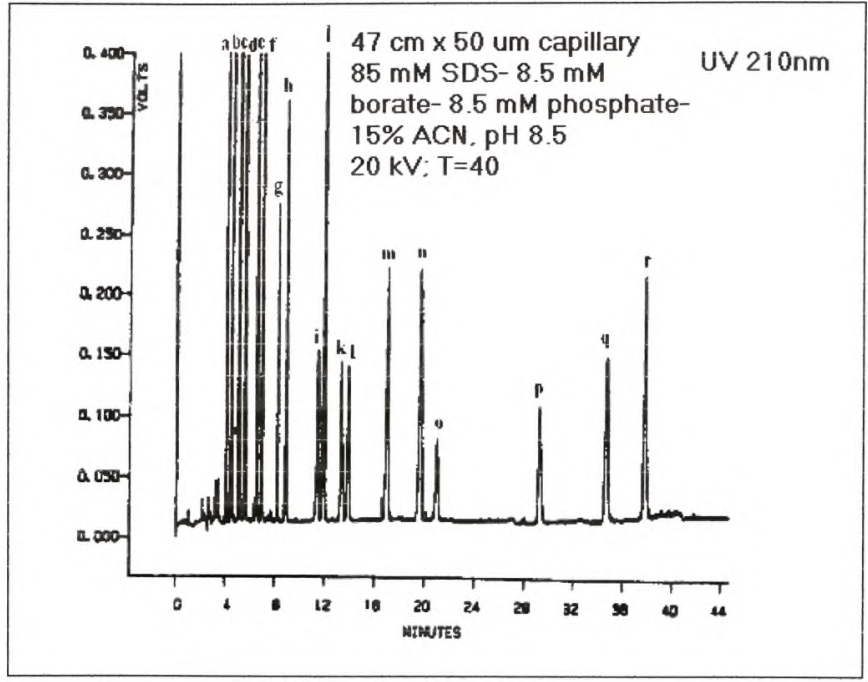

a)psilocybin b)morphine c)phenobarbital d)psilocin e)codeine f)methaqualone g)LSD h)heroin i)amphetamine j)librium k)cocaine l)methamphetamine m)lorazepam n)diazepam o)fentanyl p)PCP q)cannabidiol r) $\triangle 9-T H C$ ${ }^{*}$ Weinberger and Lurie. Analytical Chemistry 1991; 63: 823-827. ${ }^{4}$

Figure 7: Separation of 18 controlled substances, using $85 \mathrm{mM}$ SDS $/ 8.5 \mathrm{mM}$ borate $/ 8.5 \mathrm{mM}$ phosphate $/ 15 \%$ Acetonitrile. $^{4}$

Walker et al. also demonstrated a rapid procedure via MECC for the analysis of heroin samples in $1995 .{ }^{14}$ Using a system consisting of $40 \mathrm{mM}$ SDS/ $8.5 \mathrm{mM}$ borate $/ 8.5 \mathrm{mM}$ phosphate/pH 8.5, Walker et al. showed how reducing the SDS concentration and shortening the column reduced the heroin separation time to under 5 minutes, in contrast to the 14 minutes previously reported by Weinberger and Lurie in $1991 .^{14}$ 
In figure 8, Walker et al. separated an illicit sample of heroin at a concentration of $0.2 \mathrm{mg} / \mathrm{mL}$ and also containing methaqualone and noscapine. ${ }^{14}$

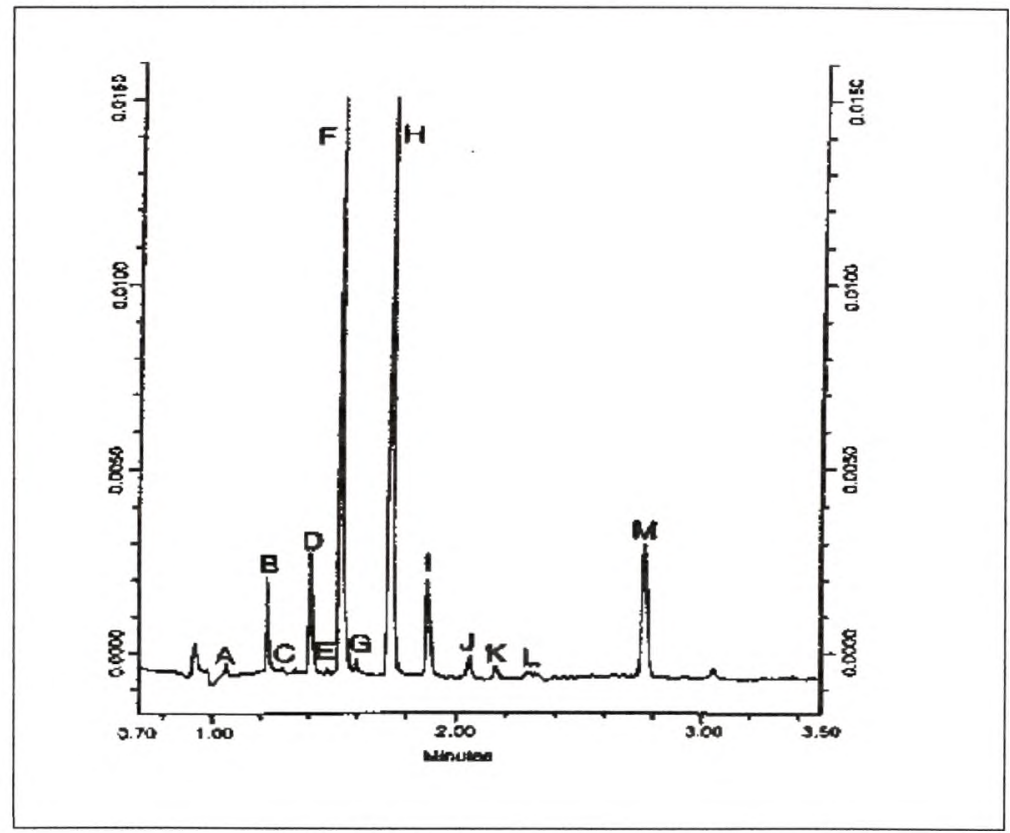

A) phenacetin B) caffeine C) morphine D) O6-monoacetylmorphine

E) codeine F) methaqualone G) phenobarbital H) NPPPB (internal standard) I) heroin J) acetylcodeine $\mathrm{K}$ ) papaverine $\mathrm{L}$ ) thebaine $\mathrm{M}$ ) noscapine

${ }^{*}$ Walker et al. J. Forensic Sci. 1995, 40, 6-9. ${ }^{14}$

Figure 8: Heroin sample separation, using $40 \mathrm{mM}$ SDS $/ 8.5 \mathrm{mM}$ borate $/ 8.5 \mathrm{mM}$ phosphate $/ 15 \% \mathrm{ACN} / \mathrm{pH} 8.5 .^{14}$ 
Using a similar MECC method, Lurie demonstrated the analysis of LSD using $53 \mathrm{mM}$ of SDS $/ 8.5 \mathrm{mM}$ borate $/ 8.5 \mathrm{mM}$ phosphate $/ 15 \% \mathrm{ACN} / \mathrm{pH} 8.5 .{ }^{15}$ Good resolution between LSD and LAMPA were obtained (figure 9). ${ }^{15}$

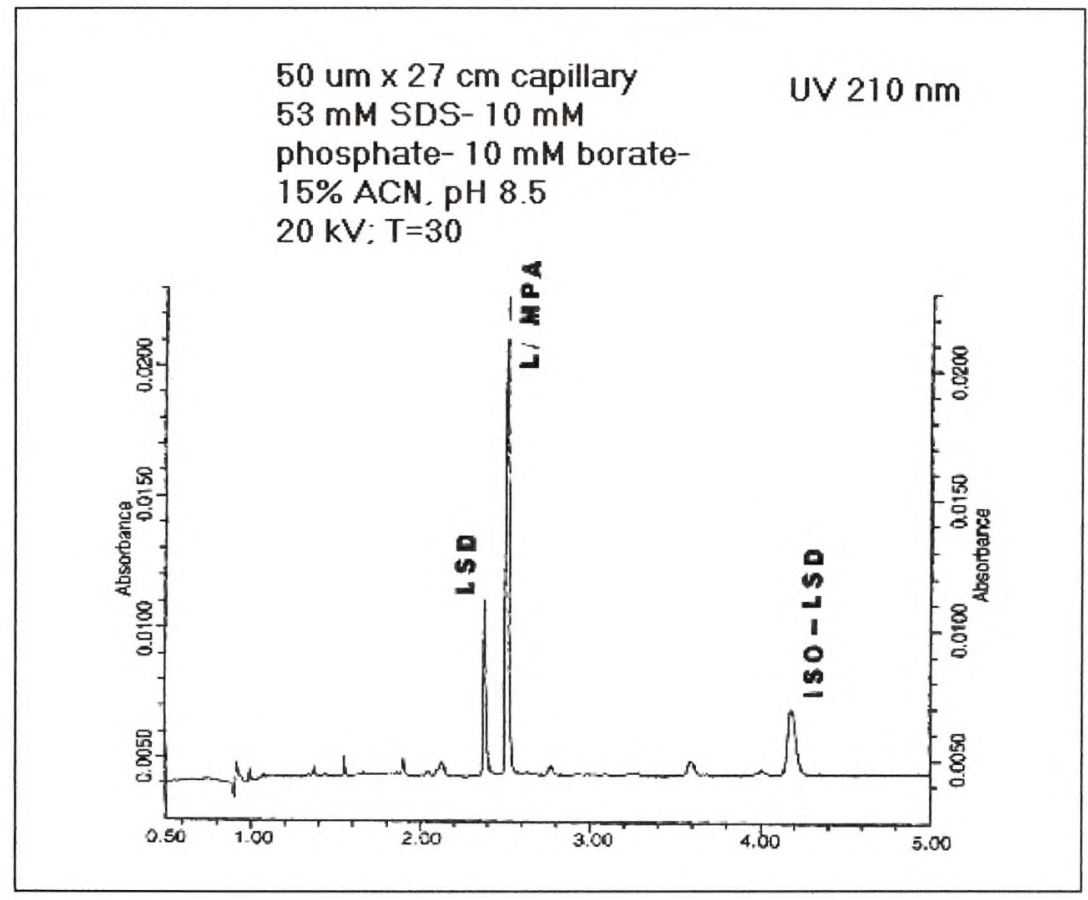

*Lurie. American Laboratory $1996 ; 28: 26-34{ }^{15}$

Figure 9: Analysis of LSD, using $54 \mathrm{mM}$ SDS/10mM phosphate/10mM borate $/ 15 \%$ Acetonitrile/pH 8.5. ${ }^{15}$ 
Forensic analysis of anabolic steroids are difficult due to their close structural similarities, poor volatility, thermal lability, low UV extinction coefficients, and the large amount of steroids. ${ }^{16}$ An MECC method was reported in 1994 for the separation of 14 anabolic steroids (figure 10). ${ }^{16}$ The separation employed the use of $75 \mathrm{mM}$ SDS in $10 \mathrm{mM}$ phosphate $/ 10 \mathrm{mM}$ borate buffer at a $\mathrm{pH}$ of $9.0 .^{16}$ Several steroid pairs were not distinguishable using this method: methandriol/stanolone , nandrolone/methandrostenolone, stanozolol/testosterone acetate, danazol/nandrolone propionate, and danazol/clostebol acetate. ${ }^{16}$ However, MECC results were comparable to GC and HPLC results. $^{16}$

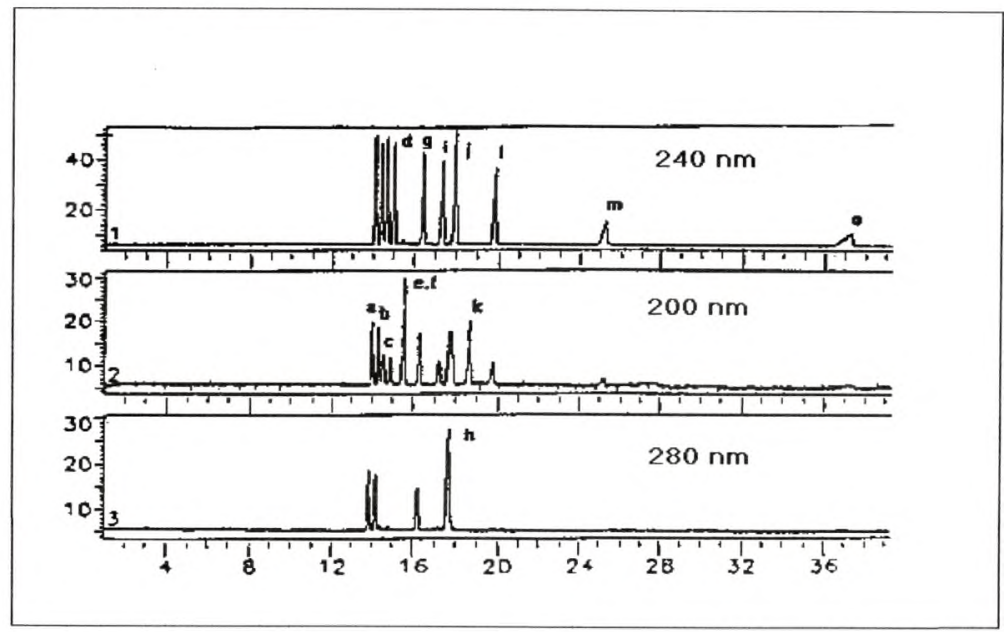

a)boldenone b)methandrostenolone c)testosterone d)methyltestosterone e)methandriol f)stanolone g)boldenone acetate $\mathrm{h}$ )danazol i)testosterone acetate j)nandrolone propionate $\mathrm{k}$ )methandriol-3-acetate 1)testosterone isobutyrate $\mathrm{m}$ )testosterone cypionate o)testosterone undecanoate *Lurie et al. J Forensic Sciences 1994; 39: 74-85. ${ }^{16}$

Figure 10: Separation of anabolic steroids using $75 \mathrm{mM}$ SDS/10mM phosphate $/ 10 \mathrm{mM}$ borate/Acetonitrile/pH 9.0. ${ }^{16}$ 
Chiral determination of abused drugs are of both legal and intelligence importance to the forensic scientist. For example, only the $(+)$ enantiomers of norpseudoephedrine and propoxyphene are controlled under federal law. Synthetic routes can also be determined by the identification of enantiomers. For example, the identification of d-methamphetamine in a sample gives strong evidence that the synthetic route was the reduction of either d-pseudoephedrine or l-ephedrine, while the identification of a racemic mixture indicates synthesis via the precursor phenylacetone.

The use of mixed cyclodextrins for chiral analysis was reported by Lurie et al. in 1994. ${ }^{17}$ Using a mixture of $5 \mathrm{mM}$ DM- $\beta-\mathrm{CD}$ and $25 \mathrm{mM}$ Tris- $\mathrm{H}_{3} \mathrm{PO}_{4}$ with $10 \%$ methanol at a $\mathrm{pH}$ of 2.45 , Lurie et al. accomplished the chiral analysis of cathinone, norpseudoephedrine, nor-ephedrine, merucathinone, merucathine, and pseudomerucathine. ${ }^{17}$ Tagliaro et. al. reported the use of $\beta$-cycodextrin for the chiral separation of 3,4-MDMA, 3,4-MDA, 3,4-MDEA, ephedrine, amphetamine, and methamphetamine in urine and hair. ${ }^{18}$

Use of dynamically coated capillaries for routine analysis of forensic drugs has been reported by Lurie et. al. in 2001. ${ }^{19}$ Using the methodology of coating the capillary column first with a proprietary polycation and then a polyanion, provided a high and reproducible EOF. In figure 11, Lurie demonstrates the power of having a high EOF through dynamic coating.for illicit cocaine samples. ${ }^{19}$ Cocaine and its alkaloids were resolved from the common cocaine adulterants of procaine, tetracaine, lidocaine, and benzocaine in less than 9 minutes. ${ }^{19}$ 


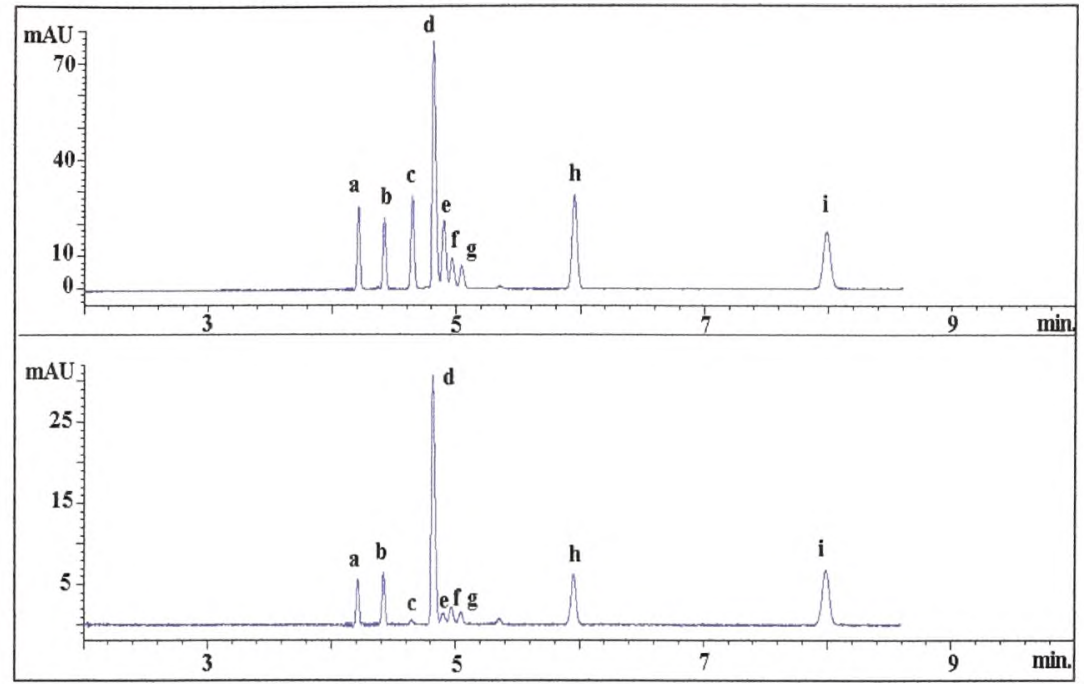

a)procaine b)tetracaine c) phenyltoloxamine (IS) d)cocaine e)lidocaine f)ciscinnamoylcocaine g)trans-cinnamoylcocaine h)benzocaine i)benzoylecgonine *Figure obtained from DEA Senior Forensic Chemist Ira Lurie. ${ }^{19}$

Figure 11: Separation of cocaine, related alkaloids and common adulterants, using a 32 $\mathrm{cm}$ x 50 um column; CE lixir buffer kit; $150 \mathrm{C} 10 \mathrm{kV} ; 195 \mathrm{~nm}$ top electropherogram and $235 \mathrm{~nm}$ bottom electropherogram. ${ }^{19}$

\section{High Performance Liquid Chromatography}

In figure 12, Ira Lurie showed how HPLC analysis can also prove to be an efficient technique for the analysis of controlled substances. Using an Hypersil ODS column with a $12.5 \mathrm{~cm} \times 4.0 \mathrm{~mm}$ ID and a $5 \mathrm{um}$ film thickness, Lurie achieved the separation of 15 compounds, consisting of controlled substances and their alkaloids. ${ }^{20}$ This separation consisted of a 20 minutes gradient with phosphate, acetonitrile, and hexylamine at a pH of 2.0 (figure 12). ${ }^{20}$ 


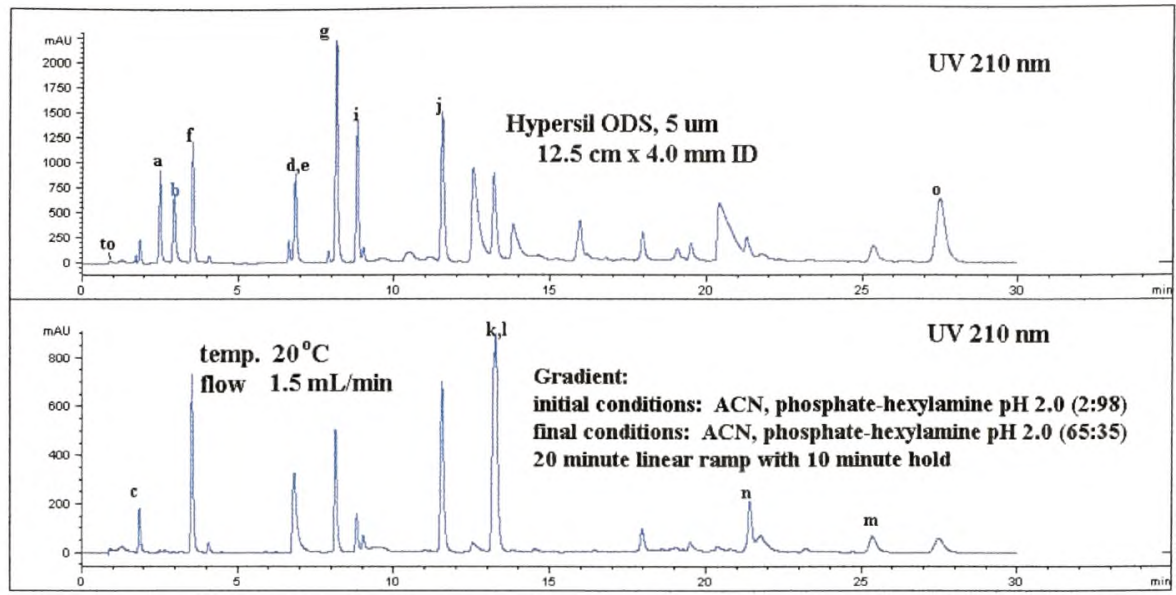
a) amphetamine
e) heroin
j) methaqualone
n) testosterone propionate
b) methamphetamine f) quinine
k) diazepam
o) $\Delta$ 9-tetrahydrocannabinol
c) procaine
g) noscapine
l) testosterone
p) $\Delta$ 9-tetrahydrocannabinolic acid
d) cocaine
i) phenobarbital
m) cannabinol

*Figure obtained from DEA Senior Forensic Chemist Ira Lurie. ${ }^{2 u}$

Figure 12: Separation of common controlled substances via HPLC, using a phosphate/ACN/pH 2.0 mobile system. ${ }^{20}$

Chiral determination of pseudoephedrine and ephedrine, using HPLC, was reported by Iwanicki et al. in 1999..$^{21}$ Using a chiral HPLC column (Supelcosil LC-(S) Naphthyl Urea), Iwanicki resolved the enantiomers after using derivatization reagents Ntrifluoroacetyl-1-prolyl chloride and 2,3,4,6-tetra-O-acetyl- $\beta$-D-glucopyranosyl. ${ }^{21} \mathrm{He}$ comapared HPLC results to CE data, resulting from the use of hydroxypropyl- $\beta-C D .^{21}$ 


\subsection{Purpose of Project}

The general aim of this project is to demonstrate the advantages of capillary electrophoresis with dynamic coating systems in drug analysis, with special emphasis on GHB and its analogue GBL, phenylethylamines, psilocybin, and benzodiazepines.

Research Questions and Hypothesis

Problem \#1:

The instrumental analysis of aqueous solutions containing GHB can be difficult due to the equilibrium of GHB with its precursor (GBL). The interconversion between GHB and GBL has been reported as a function of $\mathrm{pH}$, time, and temperature. ${ }^{22}$ GHB easily undergoes esterification, converting to the butyrolactone in the presence of small amounts of acid. Gas chromatography, a commonly employed method used for separation and quantification, can convert GHB to the lactone due to thermal reactions in a heated injection port. Current GC quantitative methods involve converting all the GHB into GBL and reporting the total content of GHB as GBL, assuming the initial concentration of GBL in the sample is negligible. ${ }^{23}$ Due to the differences in the legal scheduling where GBL is not a Schedule I substance in many jurisdictions, the intentional conversion of GHB to GBL may not present the best analytical strategy for samples that contain amounts of both GHB and GBL. Meyers and Almirall have recently reported a method, using GC/MS, for the analysis of GHB from aqueous samples without the need for manipulating the sample. ${ }^{24,25}$ Capillary electrophoresis may be a valid instrumental tool for the analysis GHB and related samples, by overcoming the interconversion problems that exist between GHB and GBL. 


\section{Hypothesis \#1:}

With the use of micelles, capillary electrophoresis can be used effectively for the separation and quantitation of the negatively charged GHB and the neutral compound GBL.

\section{Problem \#2:}

The identification and quantitation of phenylethylamines presents analytical difficulties in forensic laboratories due to their low molecular weights, polarity, and their ability to exist as optical isomers. Gas chromatography (GC) and high performance liquid chromatography (HPLC) are common techniques used to separate and quantitate many phenylethylamines. Identification of the optical isomer of methamphetamine and its precursors in forensic drug samples provides important information that the forensic scientist uses to determine the synthetic pathway utilized to manufacture the illicit methamphetamine sample. Optical isomer determination of highly pure methamphetamine samples is also of great legal importance. Federal sentencing guidelines distinguish d-methamphetamine with a purity of greater than $80 \%$ as "Ice", and associate a longer sentence for its possession or manufacture. ${ }^{26}$ For chiral separations prior to derivatization with chiral reagents (to generate diasteromers) and/or use of expensive, specialized columns is invariably required for GC and HPLC methods. Even with derivatization, the separation of all 18 optical phenylethylamine isomers is not easily accomplished in a single run, either with GC or HPLC anlalysis. Furthermore, quantitation performed by GC usually requires prior extraction. 
The determination of optical isomers using $\mathrm{CE}$ techniques requires no derivatization. In addition, CE as compared to HPLC and GC uses inexpensive capillary columns, has low solvent consumption, and employs little to none organic solvents. ${ }^{27}$ Capillary electrophoresis, through the use of charged cyclodextrins, may separate all 18 phenylethylamines.

\section{Hypothesis \#2:}

The use of highly charged cyclodextrins, such as sulfated (XIII)-gamma-cyclodextrin in capillary electrophoresis will resolve optical isomers of methamphetamine, amphetamine, norephedrine, norpseudoephedrine, ephedrine, pseudoephedrine, 3,4methylenedioxymethamphetamine (MDA), 3,4-methylenedioxyethylamphetamine (MDEA), and 3,4-methylenedioxymethamphetamine (MDMA).

\section{Problem \#3:}

The analysis of psilocybin, the psychoactive drug in illicit mushrooms, still present difficulties in forensic laboratories. The thermally degradable psilocybin breaks apart in conditions of an injection port of a gas chromatograph. Thus, routine forensic analysis identifies psilocin, the dephosphorylated form of psilocybin. ' A mushroom, which only contains psilocybin will be difficult to analyze and quantitate without prior derivatization.

\section{Hypothesis \#3:}

CZE and MECC will permit the analysis and quantitation of psilocybin without derivatization, while still remaining comparable to GC and HPLC results. 


\section{Problem \#4:}

The analysis of opium gum and latex is of great interest to the forensic community, since it contains the major alkaloids of morphine, codeine, thebaine, noscapine, and papaverine. Opium samples are currently analyzed predominantly with HPLC. HPLC methods lack resolution and exhibit poor peak shapes. In addition, HPLC columns are expensive and do not offer the high efficiency and resolution of CE methods. Will the use of dynamically coated capillary methods allow for the analysis of opium with greater resolving power than HPLC?

\section{Hypothesis \#4:}

Dynamically coated techniques in capillary electrophoresis will permit resolution over HPLC in the analysis of opium samples.

\section{Problem \#5:}

The separation and analysis of common benzodiazepines presents several challenges to the forensic scientist. Routine forensic analytical techniques do not easily differentiate between these compounds. Using infrared spectroscopy, for example, shows minimal differences in IR characteristics.

\section{Hypothesis \#5:}

Free zone capillary electrophoresis is a good separation technique for the separation and quantitation of common benzodiazepines, such as flunitrazepam and diazepam. 


\section{DRUGS OF FORENSIC INTEREST}

In the United States v. Fisher, the Eleventh Circuit held that the Analogue Act was not unconstitutionally vague as applied to gammabutyrolactone (GBL) as an analogue of gamma-hydroxybutyric acid $(G H B)$, a schedule I controlled substance because its chemical structure and effect on the central nervous system were substantially similar to GHB.

$$
\text { J. CLIC } 2004 ; 14(1): 14^{28}
$$

\subsection{Gamma-hydroxybutyric Acid (GHB)}

Gamma-hydroxybutyric acid (GHB) is a central nervous system depressant first synthesized in the 1960 s by French Physician Laborit. ${ }^{29}$ Initially introduced as an anesthetic in Europe, it was rejected among the medical community due to its side effects of hyperventilation, ataxia, and even coma. In the 1970s-80s, GHB began to be used to treat narcolepsy and alcoholism. ${ }^{29}$ Its alleged property of stimulating growth hormones, reported by Japanese scientists in 1977, led to an increased abuse of GHB by the body building community. ${ }^{30,31}$ Since the 1990s, GHB has gained popularity as a recreational drug at rave clubs and parties. GHB is commonly referred to as "G" or "Liquid X", and is often reported in conjunction with other drugs such as LSD and MDMA. ${ }^{32}$ The age groups most related to the abuse of the drug are teenagers and college students (18-25 years of age). ${ }^{33,34}$ GHB is ingested for its euphoric effects and it is reported to reduce inhibitions. Cases involving the ingestion of GHB to facilitate a sexual assault have been documented, earning the drug the label of one of the "date rape" drugs. ${ }^{35,36}$ 
<smiles>O=C(O)CCCO</smiles>

gamma-hydroxybutyric acid

(GHB)

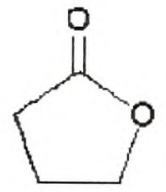

butyrolactone

Figure 13: Chemical structures of GHB and GBL.

GHB is also produced in the body as a minor metabolite of gamma-aminobutyric acid (GABA). ${ }^{37}$ In addition, the ingestion of gamma-butyrolactone (GBL) will also metabolize the drug into GHB. Figure 13 demonstrates the similarity in the two structures of GHB and GBL. GHB is most often reported as an aqueous solution or as a white powder. More recently, GHB and GBL, have been sold as dietary supplements and as commercial solvents (figure 14). ${ }^{38}$ 


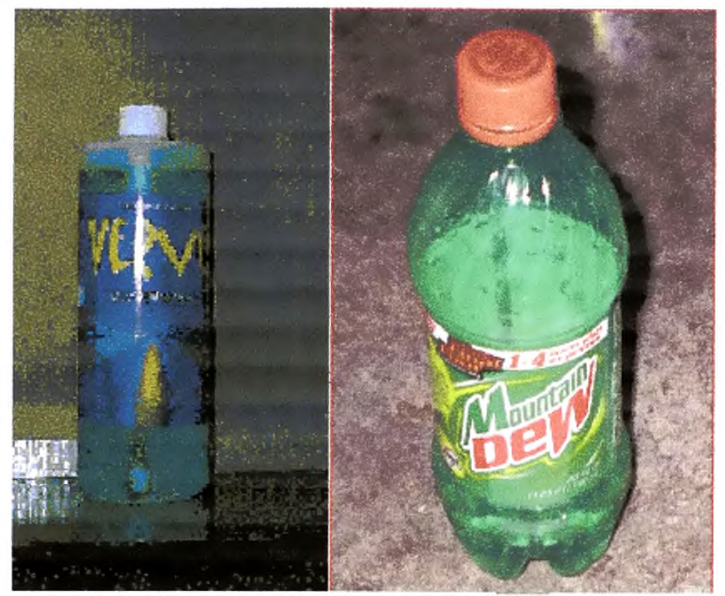

Verve bottle with $\mathrm{GBL}$ and $\mathrm{GHB}$

Mountain Dew bottle with diluted GHB and GBL

Figure 14: Picture of actual street case samples containing GHB and GBL.

Currently GHB abusers are take 1-5 grams of GHB, with one dosage costing about $\$ 10$ per capful. ${ }^{39}$ A conservative assumption would be 4.5 grams of GHB $=1$ dosage. One pound of GHB will then be equivalent to 100 dosage units, respectively. GHB is extremely easy to manufacture illicitly. It requires no chemistry expertise, no heating, and no hazardous chemicals. The only precursor needed is GBL and the only requirement is a $\mathrm{pH}$ of $8-9$, for which lye (drain opener) is most commonly used. Commonly clandestine laboratories utilize 55 gallon drums of the precursor GBL and 
buckets of sodium hydroxide (figure 15). Plastic water gallon jugs are used to dilute the sodium hydroxide. A 55 gallon drum of GBL sells through legitimate companies for approximately $\$ 1,200$, while illegitimate companies sell it for about $\$ 3,200$. A 12 kilogram bucket of sodium hydroxide sells for about $\$ 162$. A 55 gallon drum of GBL has a potential of 987.8 pounds of $\mathrm{NaGHB}$, which is equivalent to 98,780 dosage units of GHB. At \$5-10/capful (1 dose), a 55 gallon drum of GBL illicit laboratory has the potential of making $\$ 500,000$.

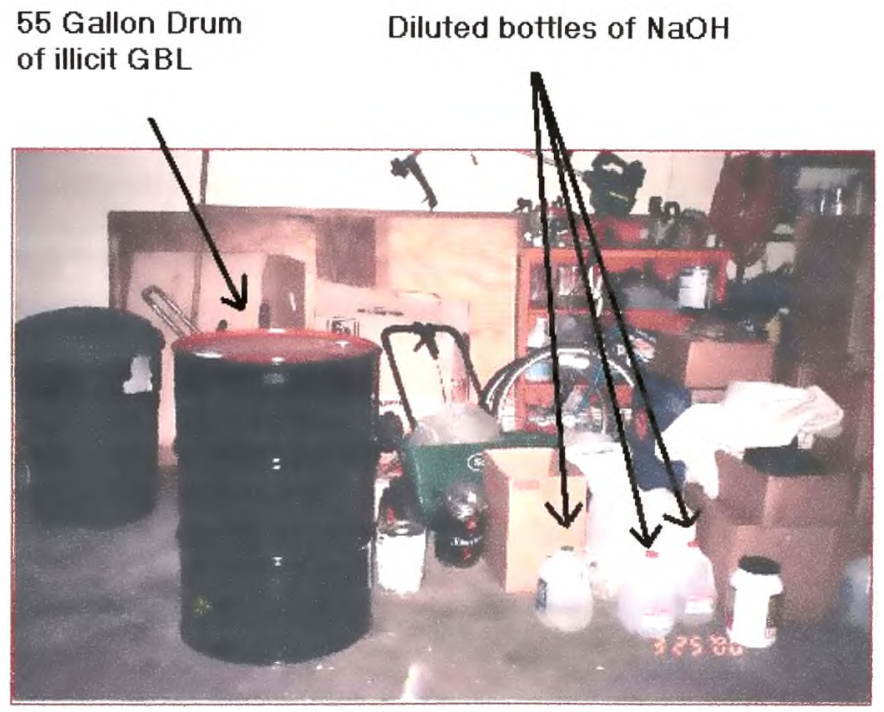

Figure 15: Clandestine laboratory depicting the illicit manufacture of GHB. 
A bill making GHB a schedule I substance was first signed into law on February $18,2000 .{ }^{38}$ Since GHB is currently being used to treat narcolepsy in 17 sleeping centers, "Medical GHB" has been designated as a schedule III and approved by FDA for treatment of narcolepsy in July of $2002 .^{40}$ The pharmaceutical formulation is marketed under the name Xyrem. ${ }^{40}$ The legislation also allows for the prosecution of the possession of the analogue GBL, under the analogue law, if this compound is "intended for human consumption". ${ }^{38}$ Due to these new developments, forensic laboratories are now required to qualitatively and, in some cases, quantitatively analyze samples with GHB and GBL.

The effects of $\mathrm{pH}$, time and temperature on the interconversion between GHB and GBL have been examined and demonstrated that at almost any $\mathrm{pH}$ interconversion will occur. ${ }^{22}$ GHB easily undergoes esterification, converting to the butyrolactone, in the presence of small amounts of acid (figure 16). In addition, conversion of GHB to the lactone results from thermal reactions (figure 17). In forensic toxicology, GC quantitative methods convert all the GHB into GBL and report the total content of GHB as GBL, while making the assumption that the initial concentration of GBL in the sample is negligible. ${ }^{23}$ Since GBL is only a Schedule I substance under the analogue law when intended for human consumption, analytical methods that employ the intentional conversion of GHB to GBL are not the best techniques for samples that contain amounts of both GHB and GBL.

Mesmer and Satzger reported in 1998 an HPLC method that consisted of diluting the sample with the mobile phase $(3.0 \mathrm{pH}$ buffer $){ }^{41}$ In light of the recent interconversion 
reports, the conditions reported for this method may lead to interconversion and may, therefore, be considered prior to use. Diluting the sample with a buffer of $\mathrm{pH}$ of $3.0^{41}$ might shift the equilibrium between GHB and GBL, in favor of GBL.

\section{Esterification of GHB to GBL}

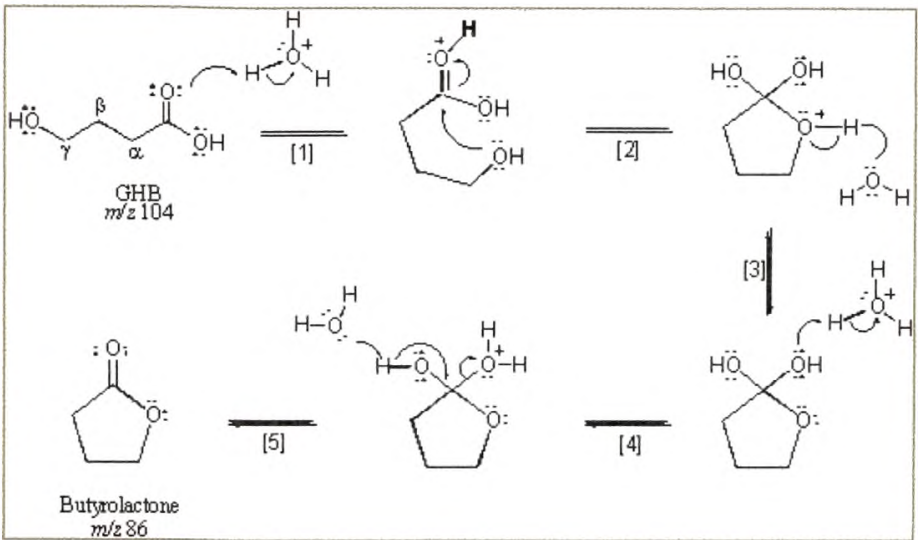

[1] proton transfer from the acid catalyst to the carbonyl oxygen

[2] ring closure by attack of the nucleophilic oxygen from hydroxyl group

[3] proton transfer from oxonium ion to a water molecule

[4] proton transfer from the acid to one of the hydroxyl group

[5] Loss of water from the oxonium ion gives the lactone

*Figure obtained from FIU Professor Dr. Almirall.

Figure 16: Esterification of GHB to GBL mechanism. 


\section{Thermal Degradation of GHB}

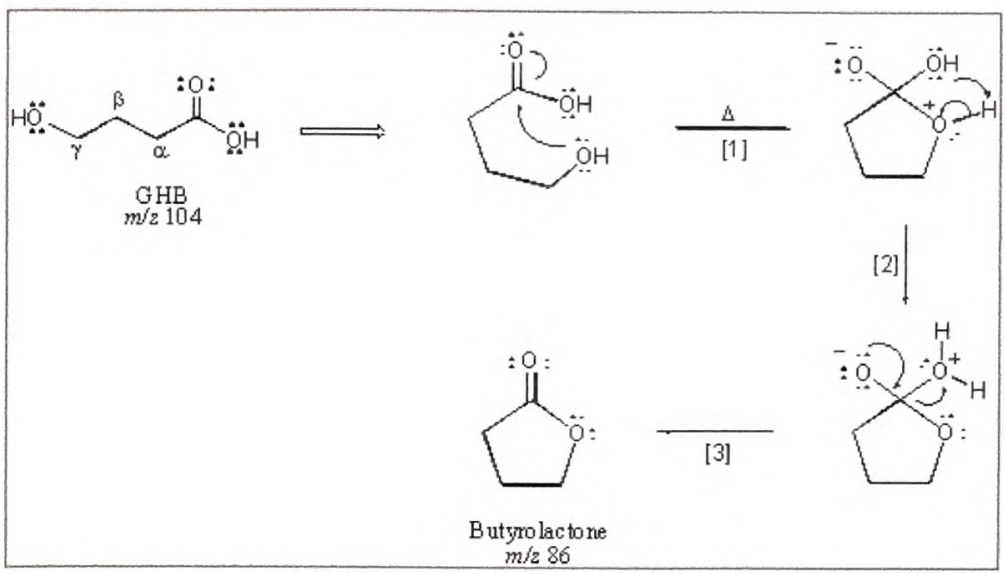

[1] ring closure by attack of the nucleophilic oxygen from hydroxyl group

[2] intramolecular proton transfer from oxonium ion to one of the hydroxyl group

[3] Loss of a water molecule

* Figure obtained from FIU Professor Dr. Almirall.

Figure 17: Thermal degradation of GHB to GBL.

The work reported in the current study maintains the $\mathrm{pH}$ at 6.5 in order to minimize interconversion, therefore minimizing the risk for the potential of acid/base interconversion that could occur at the sample preparation step. The use of a mathematical model to determine the equilibrium concentration at $\mathrm{pH} 6.5$ predicts that approximately $1 \%$ of the mixture would contain GBL. ${ }^{42}$ A previous report of a CE 
method for the analysis of GHB incorporated the use of a polyvinyl alcohol coated capillary (PVA) and a phosphate buffer. ${ }^{43}$ Separations were based on mass-to-charge ratios of the solutes with no contribution from the EOF. ${ }^{43}$ This method is effective for the analysis of GHB, but will not separate neutral compounds like GBL.

Other analytical techniques for the analysis of GHB, entail color tests, Gas Chromatograph/Mass Spectrometry (GC/MS), Solid Phase Microextraction (SPME), Fourier Transform Infrared Spectroscopy (FTIR), Attenuated Total Reflectance, and Nuclear Magnetic Resonance.

Color tests for GHB have been reported, such as 5\% Ferric Chloride, $1 \%$ Cobalt(II)Nitrate, and saturated potassium dichromate. However, low concentration GHB solutions or samples with other compounds, such as food coloring, flavoring agents, and preservatives, will not generate good, distinguishing colors.

Blackledge and Miller reported GHB analysis via GC/MS/Electron Impact in $1991{ }^{44}$ The injection of a sample of GHB without derivatization on a GC/MS, will result in a spectra of GBL due to the thermal degradation of GHB to GBL (figure 17). GHB can be derivatized using BSTFA $/ 10 \%$ TMCS - Bis(Trimethylsilyl)trifluoroacetamide + $10 \%$ Timethylchlorosilane). ${ }^{44}$ Since GBL elutes very quickly, the oven temperature should be set at a low temperature $\left(50-80^{\circ} \mathrm{C}\right)$. However, the scientist must take caution when using this derivatization agent. Excessive and prolonged use of BSTFA will deteriorate the GC column. Sample preparation entails the extraction of GHB with chloroform, followed by derivatization. GHB is only partly soluble in chloroform, thus only a portion of it will extract. SPME can be used as the sampling technique from 
aqueous solutions, eliminating the need for any extraction. A $1 \mathrm{~cm}$ sorbent-coated fiber is used to extract the GHB from the sample, and subsequently thermally desorb them into the injection port of a GC. ${ }^{24,25}$

FTIR spectra can only be obtained from clean, aqueous solutions of GHB. ${ }^{45}$ The solution can be dried at room temperature and IR can be obtained from the resulting crystals. The solution should not be heated at high temperatures, due to the thermal degradation of GHB to GBL. An alternative action could entail placing a few drops of the sample solution on a microscope slide and slowly heating it to $110-130^{\circ} \mathrm{C}$ (the melting point of $\mathrm{GHB}$ is $\left.146^{\circ} \mathrm{C}\right){ }^{46}$ The crystals can then be allowed to cool at room temperature and then in a dessicator, before the IR spectra of the resulting crystals is obtained. ${ }^{46}$ Catterton reported the analysis of GHB via ATR in $2000 .{ }^{47}$ ATR analysis can be performed by placing a few drops of the sample liquid on the diamond. The heating mantle is then set to $100^{\circ} \mathrm{C} .{ }^{47}$ Once the white flaky GHB powder appears, the spectra can be obtained. However, samples that contain GHB and a large amount of GBL will have the GHB spectra masked by the GBL.

NMR analysis of GHB was first reported by Chew. ${ }^{48}$ NMR analysis entails placing $30 \mathrm{mg}$ of the sample in $3 \mathrm{~mL}$ of deuterated water or methanol. However, dietary supplements will contain very small amounts of GHB and GBL. The low sensitivity associated with NMR analysis might be an obstacle for the analysis of dietary supplements containing GHB and GBL. 


\section{Controlled Substance Analogue Enforcement Act of 1986}

(i) the chemical structure of which is substantially similar to the chemical structure of a controlled substance in schedule I or II;

(ii) which has a stimulant, depressant, or hallucinogenic effect on the central nervous system that is substantially similar to or greater than the stimulant, depressant, or hallucinogenic effect on the central nervous system of a controlled substance in schedule I or II; or

(iii) with a respect to a particular person, which such person represents or intends to have a stimulant, depressant, or hallucinogenic effect on the central nervous system that is substantially similar to or greater than the stimulant, depressant, or hallucinogenic effect on the central nervous system of a controlled substance in schedule I or II.

Other GHB analogues include, but are not limited to, 1,4-butanediol, 4-methyl GHB, 3-methyl GHB, 4-phenyl GHB, gamma-valerolactone, and 1,4-pentanediol. ${ }^{49,50}$ The most commonly encountered analogues of GHB in forensic laboratories are GBL and 1,4-butanediol. Ingestion of 1,4-butanediol, a popular industrial solvent, causes it to convert into GHB in the body, with very toxic effects. "Clinical finding and adverse effects include vomiting, urinary and fecal incontinence, agitation, combativeness, a labile level of consciousness, respiratory depression, and death." ${ }^{.51}$ There has also been cases where addiction and withdrawal symptoms were evident. A forensic profile of 1,4butanediol was reported by Garcia and Catterton in 2002. ${ }^{52}$

\subsection{Phenylethylamines}

Amphetamine and methamphetamine, known in the streets by names such as "speed", "crank", "crystal", and "ice", are strong, synthetic stimulants. Amphetamines were first synthesized in 1887 , and were marketed as stimulants to treat low blood 
pressure in the 1930s. ${ }^{53}$ The hunger-suppressing qualities of amphetamines were highly advertised by pharmaceutical companies in the $1950-60$ s, as commercial diet pills. ${ }^{53}$ In 1962 there was an estimated 8 billion tablets produced worldwide. ${ }^{54}$ The Controlled Substance Act of 1970 made it illegal to sell or buy amphetamines, without a prescription. ${ }^{54}$ In the 1990 s biker gangs dominated the methamphetamine market in the United States.

Due to the numerous methods of manufacturing methamphetamine, law enforcement agencies have had difficulty halting the supply of methamphetamine. The most common route of synthesizing methamphetmaine involves the reduction of ephedrine (figure 18). Ephedrine is a controlled substance in the United States, so it is usually smuggled from other countries. Pseudoephedrine is also a very common precursor and easily found in over the counter cold medicine tablets. Various synthetic methods for methamphetamine use cancerous reagents and toxins, such as red phosphorous, hydrochloric acid, sulfuric acid, benzene, and acetone. ${ }^{15}$

As with other stimulants, the physiological effects of amphetamine and methamphetamine include increased heart rate, respiration, body temperature, blood pressure, energy, and appetite suppression. ${ }^{53}$ Currently, the most common routes of administration for methamphetamine are injecting and snorting. ${ }^{53}$ Since injecting methamphetamine causes pain in the blood vessels and has the risk of contaminated needles, and snorting causes irritation and pain to the nasal mucosa, smoking "ice" is becoming more and more popular. "Ife" is a street name for the recrystalization of dmethamphetamine samples, resulting in a higher purity of the drug and crystalline-like appearance. Legally "Ice" is defined as d-methamphetamine hydrochloride with a purity 
over $80 \%$ and its possession results in longer sentences. ${ }^{26}$ Regardless the route of administration, amphetamines last 4-6 hours, while smoking "ice" can produce effects lasting from 8 to 24 hours. ${ }^{53}$

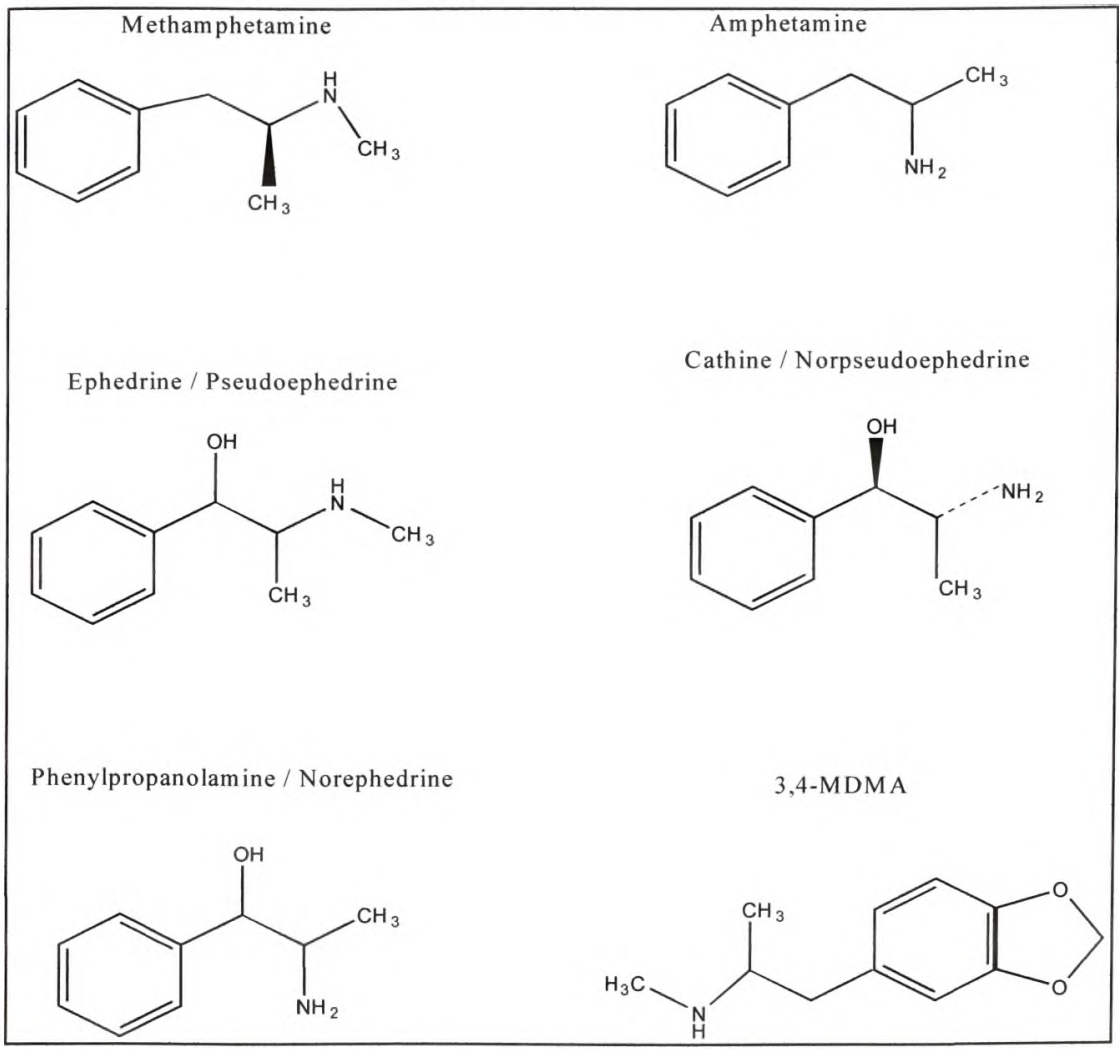

Figure 18: Chemical structures of methamphetamine and related compounds. 


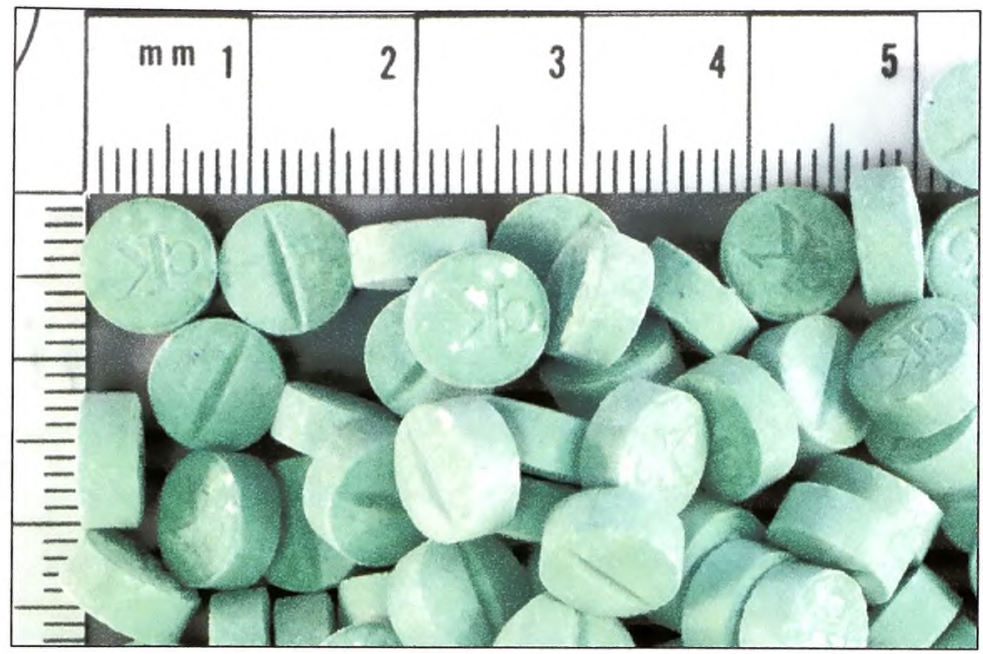

Figure 19: Picture of a typical street case of MDMA illicit tablets.

The phenylethylamines also include designer drugs of variations of methylenedioxy amphetamines. The most popular is 3,4-methylenedioxymethamphetamine (MDMA), also known in the streets as "ecstasy" and "X" (figure 19). ${ }^{54}$ MDMA is a stimulatory hallucinogen drug. Like other hallucinogens, MDMA interferes with neurotransmitters, such as serotonin (5-hydrotryptophan), and can overload the sensory pathways causing a disruption of visual and auditory centers. ${ }^{53}$ MDMA also produces stimulant effects, such as a rise in blood pressure and heart rate, excess energy and hyperactivity. ${ }^{53}$ MDMA is very popular in "Rave" clubs and parties, emerging from England and the Netherlands and spreading into the United States in the 1990s. Approximately $80 \%$ of all MDMA in the U.S. are synthesized in clandestine laboratories in the Netherlands and Belgium. ${ }^{54}$ Netherland authorities report that the average MDMA 
clandestine laboratory produces 100,000 tablets per week, with a cost of 25-50 cents each and a retail price of \$10-40 per tablet. ${ }^{54}$ The effects of the drug along with the environment in which it is taken (high physical exertion), causes an increased in body temperature inducing many deaths due to the user becoming overheated and dehydrated. ${ }^{54}$

\subsection{Psilocybin}

Mayan culture stone artifacts give evidence to psilocybin mushrooms being used by ancient Indian cultures in Mexico and Central America. ${ }^{54}$ These mushrooms were used by Indian cultures in ceremonies to contact the spirit world, as far back as 1000 B.C. ${ }^{53}$ While there are over 100 known species of mushrooms that contain psilocybin and psilocin, the Psilocybe cyanescens mushrooms are one of the more potent. ${ }^{54}$ The cyanescens mushrooms are called "wavy caps", due to their "wavy" cap (figure 20). ${ }^{54}$

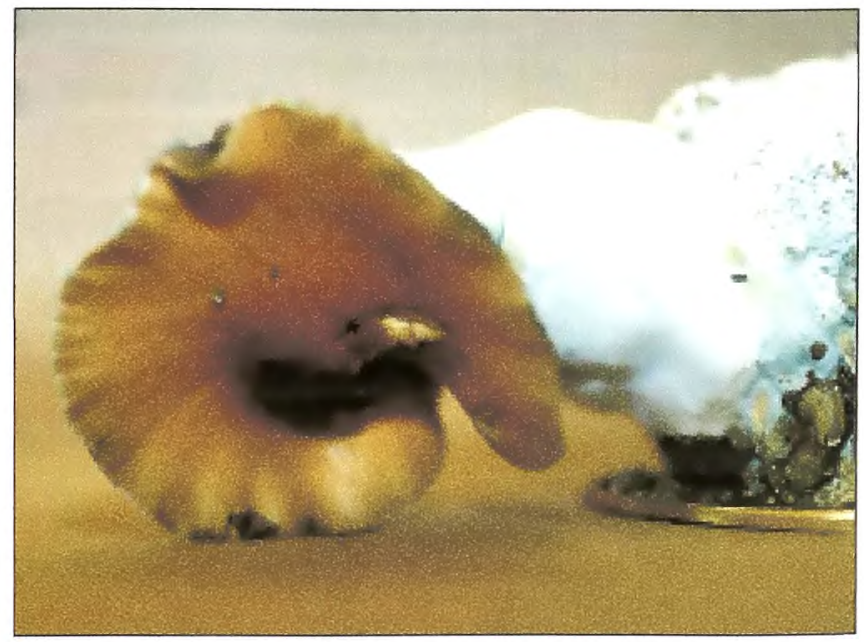

*Picture obtained from FIU Professor Dr. Almirall.

Figure 20: Picture of a Cyanescens mushroom. 
Indoor growing operations of psilocybin mushrooms are very large, due to their generation of high profits. Street prices can range from $\$ 60$ to $\$ 200$ an ounce. ${ }^{54}$ Since mushroom spores are not a Schedule I drug, like the mushroom, commercial kits to grow the psilocybin spores are readily available to consumers.

Most mushrooms found in Mexico, United States, South America, Southeast Asia and Europe contain the hallucinogenic substances psilocybin and psilocin. ${ }^{51}$ These psychedelic active ingredients of mushrooms give effects from doses of 10-60mg, and generally last 5-6 hours. ${ }^{51}$ Psilocybin breaks down into psilocin, upon ingestion. However, psilocybin "...crosses the blood-brain barrier more readily...", causing it to have double the potency as psilocin. ${ }^{51}$ Its structure is very similar to other psychedelic drugs, such as LSD (figure 21). Before the psychedelic effects of “ ...visceral sensations, changes in sight, hearing, taste, and touch, and altered states of consciousness...", psilocybin causes physical effects such as nausea. ${ }^{51}$ The hallucinogenic effects are similar to LSD and mescalin. ${ }^{51}$ 
<smiles>CP(C)CCc1cn(I)c2cccc(OP(=O)(O)O)c12</smiles>

Psilocybin<smiles>CN(C)CCc1c[nH]c2cccc(O)c12</smiles>

Psilocin

Figure 21: Chemical Structures for psilocybin and psilocin.

Analytical techniques for the analysis of psilocybin mushrooms in forensic laboratories consist predominantly of thin layer chromatography, HPLC, and IR spectroscopy. Psilocybin is highly polar and not suitable for GC analysis, unless it is derivatized. Thomson reported an HPLC method using reverse phase columns, with a mobile phase of $40: 60$ methanol/phosphate buffer $\mathrm{pH} 7.0$ to 7.5 and a $0.15 \%$ concentration of cetrimonium bromide. ${ }^{55}$ A 24 hour methanol extraction was used in this method.

\subsection{Opium}

Opium is processed from the opium poppy plant, Papaver somniferum. Poppy plants are grown in four main areas of the world: the Golden Triangle (Burma, Laos, and 
Thailand), the Golden Crescent (Afghanistan, Pakistan, and Iran), Mexico, and Colombia. ${ }^{54}$ The poppy plant can grow up to 4 feet and matures in about 90 days. ${ }^{54}$ Once the plant reaches maturity, the flower petals (most often white or red) fall and leave the green pod exposed. ${ }^{54}$ The seed pod is then incised so the opium oozes out and accumulates on the surface. The milky opium is white in color and turns dark brown within 24 hours, when it is allowed to air-dry (opium gum). The milky sap of the poppy plant can also be collected in its liquid form (opium latex). Each plant can produce an average of $80 \mathrm{mg}$ of opium, resulting in approximately $21-35$ pounds of opium per acre. ${ }^{54}$ Opium was first introduced to induce drowsiness as well as an ailment for illnesses. ${ }^{53}$ The Egyptians and the Chinese were among the ones who recorded opium as having medicinal properties, as well as being a poison. ${ }^{53}$ Opium abuse was low due to its bitter taste and low concentration of active ingredients. It the $1700 \mathrm{~s}$, smoking opium for nonmedical purposes began to become widespread. ${ }^{53}$ While opiate ingestion required 2030 minutes for the drug to reach the brain, the high concentration of the opiate obtained through smoking produced a strong sense of euphoria, relaxation, and well-being. Through smoking, the drug reaches the brain in 6-8 seconds. ${ }^{51}$ In 1848 the hypodermic needle was invented, increasing the potential for opiate abuse. ${ }^{53}$ Through intravenous use, it only takes 15-30 seconds for the opiate to affect the central nervous system of a user. $^{51}$

There are over 25 alkaloids in opium. ${ }^{54}$ The three most abundant alkaloids in opium are morphine, codeine, and thebaine. In 1806, German pharmacist Frederich refined morphine and discovered it to be 10 times more potent than opium. ${ }^{53}$ Opium extracts can used to synthesize heroin, Percodan, or Dilaudid. ${ }^{54}$ Once the opium is 
collected, it is sold to processors to extract the morphine (figure 22). The morphine is then heated with acetic anhydride, sodium chloride, and hydrochloric acid, in order to chemically synthesize heroin. Morphine is chemically converted to heroin at a one to one ratio. Over $50 \%$ of all heroin seized in the U.S. originates in Colombia with a general purity in excess of $80 \%$. ${ }^{54}$

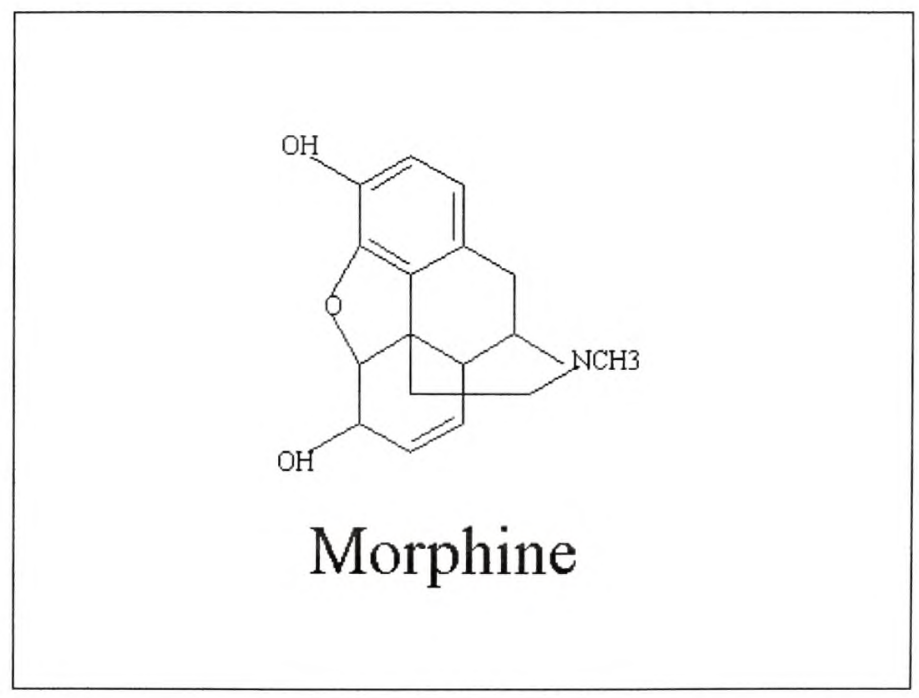

Figure 22: Chemical structure for morphine.

Most forensic laboratories analyze opium samples via HPLC. Several HPLC methods have been published on the subject. However, HPLC methods do not generally achieve good resolution between the opium alkaloids in a short amount of time. 


\subsection{Benzodiazepines}

Benzodiazepines form one of the largest classes of illegitimate preparations of abused pharmaceuticals. They are used legitimately as antiepileptics and anesthetics. Since they are central nervous system depressants with sedative and hypnotic effects, they are often abused in conjunction with other illicit controlled substances, such as methamphetamine and cocaine. ${ }^{53}$ Heroin addicts use it as an alternative to heroin, while alcoholics take them to avoid alcohol withdrawal symptoms, like convulsions. ${ }^{53}$

Overdose symptoms, include but are not limited to, drowsiness, loss of consciousness, depressed breathing, coma, and even death. Tolerance develops after long term use, depending on which benzodiazepine.

Common abused benzodiazepines are diazepam and flunitrazepam. Even though other countries use flunitrazepam to treat insomnia, FDA has not approved its use in the United States. Due to its strong sedative properties, flunitrazepam has been associated in cases of date rape and sexual assault. Thus, flunitrazepam has been included in the Drug Induced Rape Prevention Act of $1996 .{ }^{54}$ Rohypnol in the United States is usually smuggled from Mexico or Colombia. To attempt reducing the use of Rohypnol in rape cases, the manufacturer reformulated Rohypnol to release a blue dye once introduced to a liquid. $^{54}$

Counterfeit tablets generally contain controlled substances and are designed to be sold in the illegitimate or clandestine market. The most common counterfeit tablets are diazepam tablets, which usually resemble legitimate pharmaceutical preparations, such as Valium ${ }^{\circledR}$ tablets. ${ }^{54}$ Other common abused benzodiazepines that show up in forensic laboratories as counterfeit, include flunitrazepam (Rohypnol®) and alprazolam 
(Xanax $($ )). Most abusers, however, obtain benzodiazepines through prescriptions (figure 23).

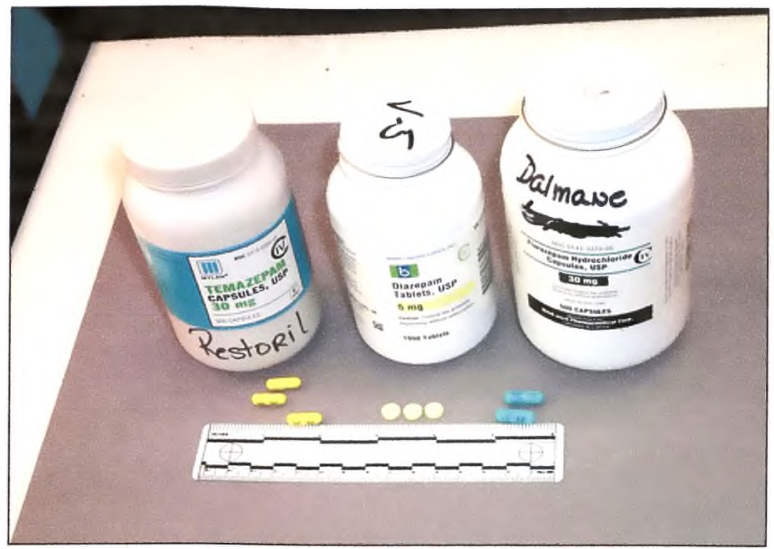

Figure 23: Typical benzodiazepine samples: temazepam, diazepam, and flurazepam.

Benzodiazepines consist of lipophilic acids with low pKa's. Diazepam, for example, has a pKa of 3.4. Thus, diazepam is absorbed quickly through oral administration. ${ }^{51}$ The fast absorption rate of diazepam (1 hour for adults and 15-30 minutes for children) is explained by the lipophilicity of diazepam. ${ }^{51}$ Less lipophilicity benzodiazepines have slower absorption rates. ${ }^{51}$ Refer to figure 24 for chemical structures of common benzodiazepines. 


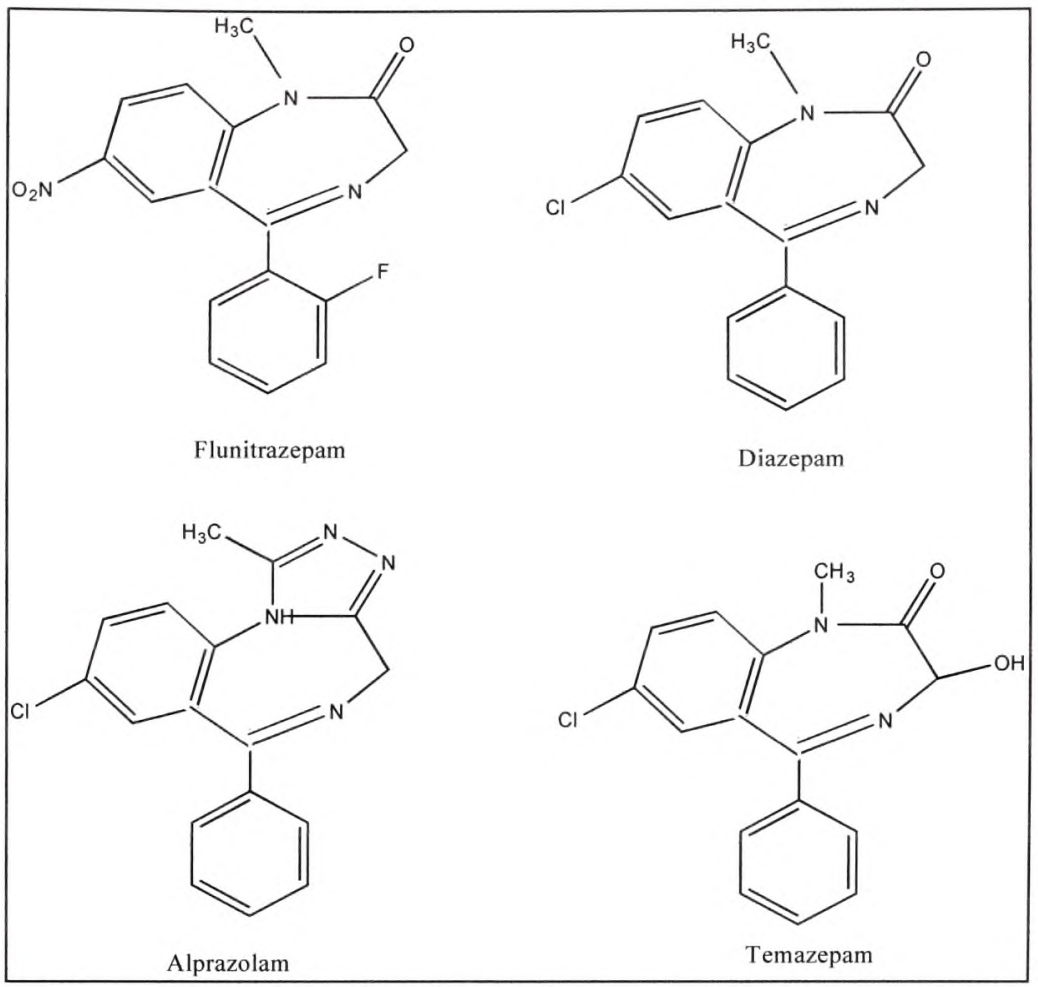

Figure 24: Chemical structures for typical benzodiazepines, flunitrazepam, diazepam, alprazolam, and temazepam. 


\section{CE METHODOLOGY}

\subsection{GHB Method}

A Hewlett Packard 3D Capillary Electrophoresis (Agilent Technologies) and a Beckman P/ACE System 5000 electropherograph (Fullerton, CA, USA) were used for this study. Both instrument systems used bare fused silica capillaries of different lengths and diameters, along with CE extended light path columns, of $50 \mu \mathrm{m}$ internal diameter and $48.5 \mathrm{~cm}$ in length. A Photo Diode Array (PDA) detector was used to scan the range of $190-440 \mathrm{~nm}$ and set to $195,200,205$, and $210 \mathrm{~nm}$ wavelengths with a $20 \mathrm{~nm}$ bandwidth for the determinations. Refer to figure 25 for the ultraviolet spectra for GHB and GBL.

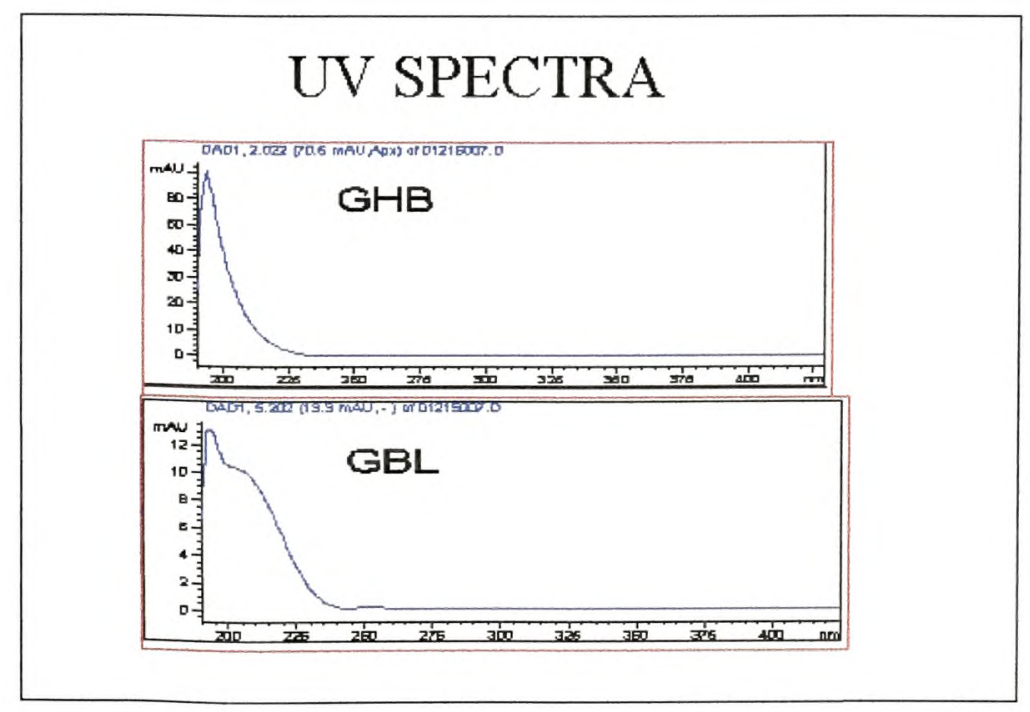

Figure 25: UV spectra for GHB and GBL. 
Sample injection was achieved via hydrodynamic injection at pressures of $0.5 \mathrm{psi}$ for 4 seconds and $25 \mathrm{mbar}$ for 3 seconds for the Beckman and Agilent systems, respectively. The voltage and temperature were optimized for each instrument system and the operating parameters for each are described below. Phosphate and borate running buffers with either SDS or CTAC were used. Ascorbic acid and l-histamine were used as internal standards along with external calibration curves for quantification calculations.

Electrophoresis grade sodium dodecyl sulphate (SDS) and sodium borate, both of $<99 \%$ purity, were purchased from Fisher Scientific (New Jersey, USA). HPLC grade water (Fisher Scientific) was used to make the buffer systems and high purity deionized water $\left(>18 \mathrm{M} \Omega \mathrm{cm}^{-1}\right)$ was obtained with a Nanopore Infinity purification system (Barnstead, Dubuque, IA, USA). Sodium phosphate, along with Potassium phosphate, of $<99 \%$ purity was purchased from Sigma Chemical Company (St Louis, USA). Cetyltrimethylammonium chloride (CTAC), $25 \%$ by weight in water was supplied by Aldrich Chemical Company, Inc, Milwaukee, USA. The pHs of the buffers were modified using either $1 \mathrm{M}$ sodium hydroxide (Fisher Scientific), $1 \mathrm{M}$ hydrochloric acid (Fisher Scientific), or concentrated phosphoric acid (Fisher Scientific). All prepared solutions were filtered using $0.45 \mu \mathrm{m}$ polypropylene filters or $13 \mathrm{~mm} \mathrm{GD} / \mathrm{X}$ disposable filters with $45 \mu \mathrm{m}$ pore size supplied by Whatman ${ }^{\circledR}$ (New Jersey, USA).

GHB and GBL standards were obtained from Aldrich. The internal standards, ascorbic acid and histamine, used in the CE systems were obtained from Eastman Organic Chemicals (Kingsport, TN). CE capillaries were obtained from Polymicro Technologies (Arizona, USA), while CE extended light path columns were obtained from Agilent Technologies. 


\subsection{Phenylethylamines}

A cyclodextrin system was examined on a Hewlett Packard 3D HP Capillary Electrophoresis for the chiral analysis of all 18 phenylethylamines. The buffer system consisted of $10 \mathrm{mM}$ gamma-highly sulfated cyclodextrin $/ 50 \mathrm{mM}$ Phosphate buffer at a $\mathrm{pH}$ of 7.0. ${ }^{56}$ Another dynamic coating system was explored using $10 \mathrm{mM}$ gamma-highly sulfated cyclodextrin $/ 50 \mathrm{mM}$ Phosphate buffer at a $\mathrm{pH}$ of 2.6 , with reversed polarity. ${ }^{56}$ The columns utilized consisted of $32-49 \mathrm{~cm}$ in length and a $52 \mathrm{~cm}$ in internal diameter. Temperature and applied voltage was explored at different settings, while sample introduction consisted of hydrodynamic injections of $50 \mathrm{mbar} / \mathrm{second}$.

All samples were prepared by dissolving them in 50mM Phosphate Buffer pH 7.0 solution, which contained $0.5 \mathrm{mg} / \mathrm{mL} \mathrm{n}$-butylamphetamine. Standards were prepared by accurately weighing a standard amounts treating them in the same manner as the samples. Subsequently, filtering them through a 0.45 um polypropylene filter. Stock standards were prepared in methanol or methylene chloride.

Phenylethylamine standards were obtained from the DEA Special Testing and Research Laboratory. The internal standard, n-butylamphetamine, was synthesized by Senior Forensic Chemist Timothy McKibbens from the DEA Special Testing and Research Laboratory. CE capillaries were obtained from Polymicro Technologies (Arizona, USA).

Dynamic coating methods entailed the use of Celixir buffer kits. Capillary columns of $32 \mathrm{~cm} \times 50 \mathrm{um}$ were used, with a temperature of $15^{\circ} \mathrm{C}$ and an applied voltage of $10 \mathrm{kV}$. 


\subsection{Psilocybin}

Two CE modes were explored for the analysis of the Cyanesce mushroom. Free Zone Capillary Electrophoresis was achieved utilizing a Beckman P/ACE System 5000 series, equipped with a UV detector set at a wavelength of $254 \mathrm{~nm}$. A $57 \mathrm{~cm} \times 25 \mathrm{um}$ fused silica capillary column was used. The background electrolyte consisted of $10 \mathrm{mM}$ Borate/10mM Phosphate Buffer at a $\mathrm{pH}$ of 11.5 , as previously reported. ${ }^{57}$ The temperature was set at 25 degrees Celsius, while a hydrodynamic injection was carried out (approximately $0.2 \mathrm{nl}$ ) and a voltage of $8 \mathrm{kV}$ was applied.

MECC was also examined using a Hewlett Packard 3D CE, equipped with a diode array detector. The wavelength was set at $210 \mathrm{~nm}$ and a $50 \mathrm{~cm} \times 50 \mathrm{um}$ capillary was utilized. The background electrolyte consisted of $85 \mathrm{mM} \mathrm{SDS} / 8.5 \mathrm{mM}$ Phosphate $/ 8.5 \mathrm{mM}$ Borate $/ 15 \%$ Acetonitrile at a $\mathrm{pH}$ of 8.5 , as previously reported by Lurie et al. ${ }^{4}$ Temperature was kept constant at 25 degrees Celsius, while a hydrodynamic injection of $50 \mathrm{mbar} / 1 \mathrm{sec}$ was carried out. The applied voltage was $12 \mathrm{kV}$, respectively.

Psilocybin and psilocin standards were obtained from the DEA Special Testing and Research Laboratory, the Southeast Laboratory, and the International Forensic Institute from the Florida International University. CE capillaries were obtained from Polymicro Technologies (Arizona, USA) and from the Internation Forensic Institute at the Florida International University.

Standard samples were prepared using $1 \mathrm{mg}$ of psilocybin in $10 \mathrm{~mL}$ of methanol for a final concentration of $100 \mathrm{ppm}$. Standard dilutions were then prepared for linearity 
analysis. Both psilocybin and psilocin standards were obtained from the Drug Enforcement Administration, Southeast Laboratory in Miami, Florida.

Mushroom samples were weighed and extracted in an ultrasonic bath for 15 minutes with $5 \mathrm{~mL}$ of methanol. This extraction procedure was repeated 3 times, to ensure complete extraction of psilocybin from the mushroom. The extracts were then analyzed, using pressure injection and an external standard methodology.

\subsection{Opium}

Using a capillary column of $50 \mathrm{um}$ ID with a $32 \mathrm{~cm}$ length, a buffer system consisting of $25 \mathrm{mM}$ hydropypropyl- $\beta$-cyclodextrin (HP-CD) $+75 \mathrm{mM}$ dimethyl- $\beta$ cyclodextrin (DM-CD) added to the CElixir accelerator solution B pH 2.5 was utilized. ${ }^{58}$ The temperature was maintained at $15^{\circ} \mathrm{C}$, while $20 \mathrm{kV}$ was applied. Three different wavelengths were monitored: $205 \mathrm{~nm}, 252 \mathrm{~nm}$, and $310 \mathrm{~nm}$.

Opium samples were prepared by weighing $100 \mathrm{mg}$ of opium sample into $50 \mathrm{~mL}$ volumetric flask, pipeting $5.0 \mathrm{~mL}$ of internal standard solution and diluting to volume with phosphate buffer. Samples had to be sonicated for 30 minutes at $50-60^{\circ} \mathrm{C}$ prior to the addition of the internal standard. The addition of $25 \mathrm{~mL}$ of methanol helped the opium go into solution. The internal standard consisted of $159 \mathrm{mg}$ of tetracaine hydrochloride into $100 \mathrm{~mL}$ of 50:50 mixture of methanol and $3.75 \mathrm{mM}$ phosphate buffer $\mathrm{pH} 3.2$.

All standards were obtained from Special Testing and Research Laboratory. CE capillaries were obtained from Polymicro Technologies (Arizona, USA). 


\subsection{Benzodiazepines}

A Hewlett Packard 3D Capillary Electrophorsis was utilized, along with a $48.5 \mathrm{~cm}$ length, $50 \mathrm{um}$ id, and a $40 \mathrm{~cm}$ effective length fused silica capillary column. The optimized method used 50mM Phospate buffer pH 2.5 for free zone capillary electrophoresis. The column compartment's temperature was set to $25^{\circ} \mathrm{C}$, with an applied voltage of $27 \mathrm{kV}$. The diode array detector was set to monitor a wavelength of $210 \mathrm{~nm}$.

Standard samples were prepared using benzodiazepine standards obtained from the Drug Enforcement Administration, Southeast Laboratory in Miami, Florida. Standard solutions of diazepam and flunitrazepam were accurately weighed and prepared in $1 \mathrm{~N} \mathrm{HCl}$ solution, which contained the internal standard tetracaine at $0.2 \mathrm{mg} / \mathrm{mL}$. Standard dilutions were then prepared for linearity analysis. All solutions were made fresh and sonicated for approximately 10-15 minutes, to ensure complete dissolution. 


\subsection{HPLC METHODOLOGY}

\subsection{GHB}

A Hewlett Packard 1100 HPLC was used, along with two C18 Aqua (Phenomenex) columns of lengths $(150 \times 4.6 \mathrm{~mm}$ and $250 \times 4.6 \mathrm{~mm})$. A mobile phase of $100 \% 25 \mathrm{mM}$ Potassium Phosphate of $\mathrm{pH} 6.5$ was utilized for GHB quantifications. An organic modifier (70\% 25mM Potassium Phosphate Buffer $\mathrm{pH} 6.5$ )/30\% Methanol) was introduced for GBL quantifications using the longer column in order to shorten the time of analysis. The temperature of the columns were kept constant at $25^{\circ} \mathrm{C}$. A Photo Diode Array (PDA) detector was used to scan the range of 190-440 nm and set to 195, 200, 205, and $210 \mathrm{~nm}$ wavelengths with a $10 \mathrm{~nm}$ bandwidth for the determinations. Refer to figure 18 for the ultraviolet spectra for GHB and GBL. An isocratic program with a solvent flow rate of $1.0 \mathrm{ml} / \mathrm{min}$ was employed, along with $1.5 \mu \mathrm{L}$ injections of the sample. External calibration curves were used for quantification calculations. The HPLC C18 Aqua columns $(250 \times 4.6 \mathrm{~mm}, 200 \mathrm{~A}, 5 \mu)$ and $(150 \times 4.6 \mathrm{~mm}, 200 \mathrm{~A}, 5 \mu)$ were provided by Phenomenex (Torrance, CA).

Standards for the HPLC were prepared at different concentrations of GHB and GBL, and dissolved in HPLC water. Standards for the CE determinations were prepared at different concentrations of GHB and GBL, and dissolved in HPLC water or buffer containing the corresponding internal standard. Calibration standard solutions ranged in concentration from $0.32 \mathrm{mg} / \mathrm{ml}$ to $15.3 \mathrm{mg} / \mathrm{ml}$ and the samples were diluted to a final concentration of GHB and GBL approximately equivalent to that of the standard solution. Samples were then diluted to volume with HPLC water or buffer with or without the internal standards. Aliquots of the samples and standards were then filtered with $0.45 \mu \mathrm{m}$ 
polypropylene filters or $13 \mathrm{~mm} \mathrm{GD/X} \mathrm{disposable} \mathrm{filters.} \mathrm{A} \mathrm{series} \mathrm{of} \mathrm{actual} \mathrm{casework}$ samples for the quantification experiments were taken from many different matrices, including RenewTrient, Blue Nitro, Verve, and aqueous solvents (figures 14).

\subsection{Phenylethylamines}

An 1100 Hewlett Packard HPLC, equipped with a diode array detector was utilized. A 210nm wavelength was used with an octadecyl silane (ODS) C18 column of 5um stationary phase and $125 \times 4 \mathrm{~mm}$ length. The mobile system consisted of a $2.5 \mathrm{mM}$ Phosphate $\mathrm{pH} 2.5$ buffer, with a gradient. Initial conditions were $85 \%$ buffer with a $15 \%$ acetonitrile, while final conditions consisted of $40 \%$ acetonitrile and $60 \%$ buffer. Temperature was maintained constant at $45^{\circ} \mathrm{C}$.

Gas liquid chromatography results were also obtained, using a Hewlett Packard $6890 \mathrm{GC}$ equipped with a 30 meter HP-5 column. The injection port was maintained at $265^{\circ} \mathrm{C}$, while the run consisted of a temperature program of $135^{\circ} \mathrm{C}$ to $150^{\circ} \mathrm{C}$ with a split of 100 to 1.

\subsection{Psilocybin}

An 1100 Hewlett Packard HPLC, equipped with a diode array detector was utilized. A 254nm wavelength was used with an octadecyl silane (ODS) C18 column of 5um stationary phase and $125 \times 4 \mathrm{~mm}$ length. The mobile system consisted of a gradient of methanol and acetonitrile. Other LC experiments were completed by FIU graduate student Sue Gross, as part of collaboration project, which compared CE results with LC results. An LC equipped with an electron capture detector (ECD) was used. 
Psilocybin and psilocin standards were obtained from DEA Southeast Laboratory and the International Forensic Institute from the Florida International University. HPLC columns were obtained from the Southeast Laboratory and Florida International University.

\subsection{Opium}

An 1100 Hewlett Packard HPLC, equipped with a diode array detector was utilized. Three wavelength were used, $210 \mathrm{~nm}, 228 \mathrm{~nm}$, and $240 \mathrm{~nm}$ respectively. Alkaloids were separated on a partisil 5 octadecyl silane (ODS-3) column of 5 um stationary phase and $3.2 \mathrm{~mm} \times 12.5 \mathrm{~cm}$ length. The mobile system consisted of $5 \%$ methanol/95\% phosphate buffer at a pH of 2.2. A gradient was used to a final concentration of $80 \%$ methanol, and then switching the solvents $5 \%$ acetonitrile/95\% phosphate buffer $\mathrm{pH} 2.2$.

All alkaloid standards and HPLC columns were obtained from DEA Special Testing and Research Laboratory. 


\subsection{CE RESULTS AND DISCUSSION}

\section{$5.1 \mathrm{GHB}$}

Since GHB is a very polar compound (figure 13), its interaction with the micelles is based on charge attraction. SDS is an anionic micelle with a large negative charge surface. This negative charge imparts a high electrophoretic mobility toward the positive electrode, slowing the rate in which the anionic micelles migrate towards the cathode. The micelles are still carried towards the cathode due to the presence of EOF (figure 3). CTAC, on the other hand, is an alkylammonium salt that reverses the flow of the EOF towards the anode. It is a positively charged micelle that will in turn attract anionic components, like GHB. Both of these electrophoretic systems were examined for utility in separation and quantification of GHB and GBL in aqueous samples.

Since both GHB and GBL contain weakly absorbing chromophores, detection was performed at $200 \mathrm{~nm}$. Phosphate and borate buffers were chosen due to their low absorbance at the wavelength of detection. The concentration of SDS was varied to reduce separation analysis times. The normal order of migration using an SDS system is anions first, neutrals next, and cations last. Using the SDS micelle system, GHB migrated first. This is due to GHB being an anion and the electrostatic repulsion between anions and the negatively charged SDS micelles. GBL, a neutral molecule, was separated based on its hydrophobicity. Lurie et al. employed a buffer comprising $85 \mathrm{mM} \mathrm{SDS}$, $85 \mathrm{mM}$ borate, $85 \mathrm{mM}$ phosphate and $15 \%$ acetonitrile at $\mathrm{pH} 8.50$, successfully separating a variety of controlled substances. ${ }^{4}$ Using a similar system consisting of $50 \mathrm{mM}$ 
$\mathrm{SDS} / 25 \mathrm{mM}$ phosphate $/ 25 \mathrm{mM}$ borate at $\mathrm{pH} 8.50$, GHB migrated at $16.07 \mathrm{~min}$ and $\mathrm{GBL}$ at 30.77 minutes (figure 26).

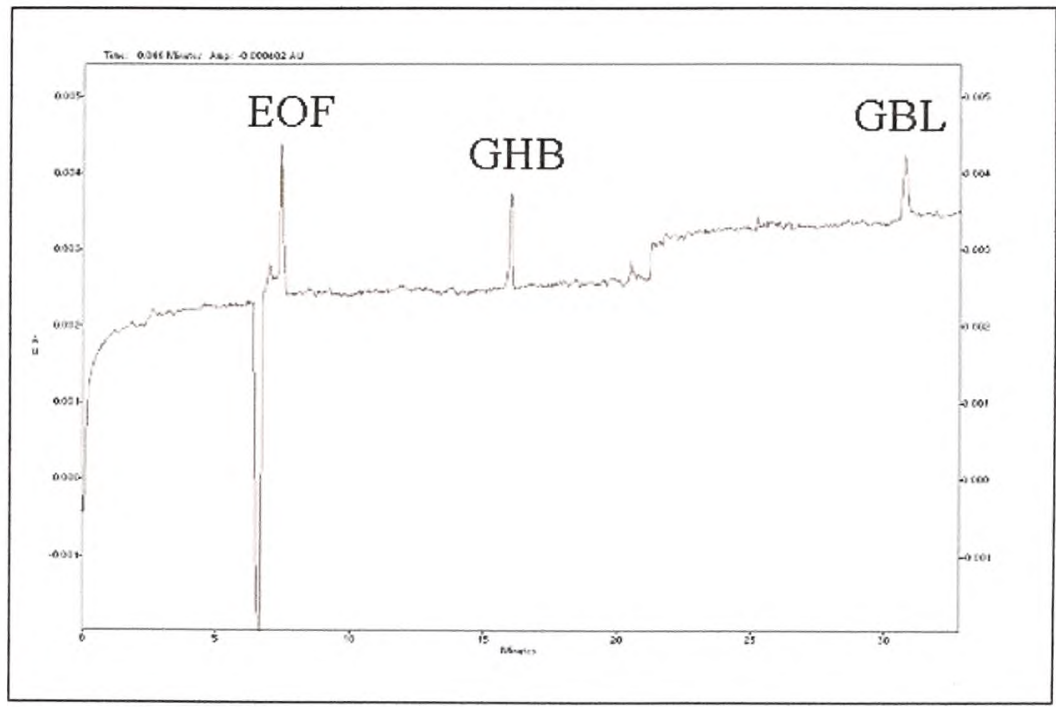

*Electropherogram obtained from FIU student Lucy Hulett.

Figure 26: The separation of GHB (16.07 minutes) and GBL (30.77 minutes), using $50 \mathrm{mM}$ SDS $/ 25 \mathrm{mM}$ phosphate $/ 25 \mathrm{mM}$ borate at $\mathrm{pH} 8.50$ on a Beckman P/ACE System $5000 \mathrm{CE}$.

Increasing the $\mathrm{pH}$ causes the silanol charge density on the capillary wall to increase along with the EOF. ${ }^{3}$ Although migration times for GHB and GBL were shorter (15.15 $\mathrm{min}$ and $25.93 \mathrm{~min}$, respectively) at higher $\mathrm{pH}$, baseline noise also increased.

Separation at elevated temperature also caused a decrease in migration times and allowed for separation of both compounds within 22 minutes at $50^{\circ} \mathrm{C}$. When a shorter column was used $(37 \mathrm{~cm})$, migration times of GHB and GBL were reduced to 5.94 and 10.86 minutes, 
respectively. This system, however, is not suitable due to the problem of interconversion of GBL to GHB at high $\mathrm{pH}$.

The cationic surfactant chosen for this system was cetyltrimethlyammonium chloride (CTAC). The addition of CTAC to the buffer caused a reverse in the polarity, which in turn reversed the EOF. Addition of this surfactant changed the migration order to cations, neutrals, and then anions. Anionic components are attracted to the positively charged micelles, and thus are better retained. Since the goal at this point was to reduce the $\mathrm{pH}$, the buffer comprised of only $50 \mathrm{mM}$ CTAC and $25 \mathrm{mM}$ phosphate for a final $\mathrm{pH}$ of 6.4. GBL migrated at 2.82 minutes and GHB at 1.86 min (figure 27). The resolution between GHB and GBL

calculated by $\mathrm{R}=2(\mathrm{t} 2-\mathrm{t} 1) / \mathrm{w} 1+\mathrm{w} 2$, where $\mathrm{t}=$ migration time and $\mathrm{w}=$ baseline peak width $^{5}$

was 6.11, while the resolution between GBL and 1-histidine was 2.25. There was a linear response between concentration and peak area for GHB from $0.039 \mathrm{mg} / \mathrm{ml}$ to 10.01 $\mathrm{mg} / \mathrm{ml}$, and had a correlation coefficient of 0.9999 . For GBL, the data produced a linear response from $1.30 \mathrm{mg} / \mathrm{ml}$ to $10.37 \mathrm{mg} / \mathrm{ml}$ with similar correlation coefficients. Alphahydroxybutyric acid and beta-hydroxybutyric acid were also evaluated using this system and migrated at similar times to GHB. 


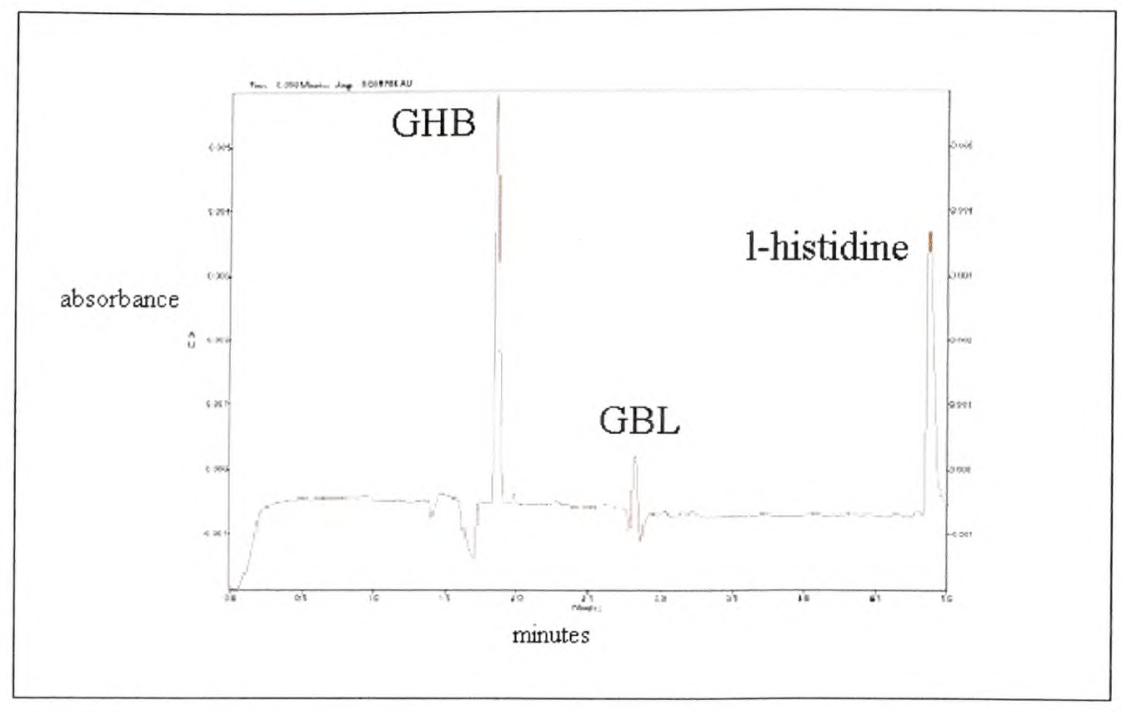

*Electropherogram obtained from FIU graduate student Lucy Hulett.

Figure 27: GHB (1.86 minutes), GBL (2.82minutes), and internal standard l-histidine (4.9 minutes), using $50 \mathrm{mM} \mathrm{CTAC}$ and $25 \mathrm{mM}$ phosphate for a final $\mathrm{pH}$ of 6.4 on a Beckman P/ACE System $5000 \mathrm{CE}$.

Since neutral compounds were not differentiated, use of this method is not recommended for the optimum quantitation of GHB (anion) and GBL (neutral) samples. Increasing the amount of CTAC in the buffer system could increase the amount of current generated, and hence potentially cause joule heating and poor reproducibility. However, this was not the case when the CTAC concentration was increased to $300 \mathrm{mM}$ (figure 28). 


\section{Ohm's Law Plot}

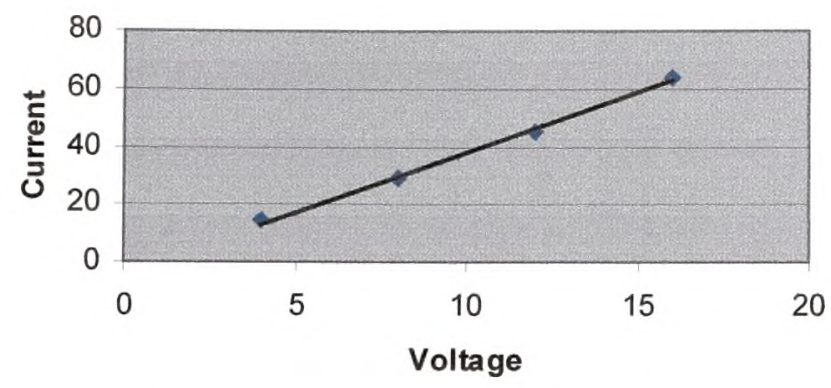

Figure 28: Ohms' Law Plot for GHB, using 25mM Potassium Phosphate/300mM CTAC pH 6.3 /CE Extended Light Path Column on an Hewlett Packard 3D CE.

Using the increased CTAC concentration and optimal temperature and voltage conditions (described in the methods section), permitted the migration of GHB at 3.9 minutes and GBL at 5.3 minutes (figure 29). A neutral marker (caffeine) migrated at 5.0 minutes, using the higher CTAC concentration of $300 \mathrm{mM}$. 


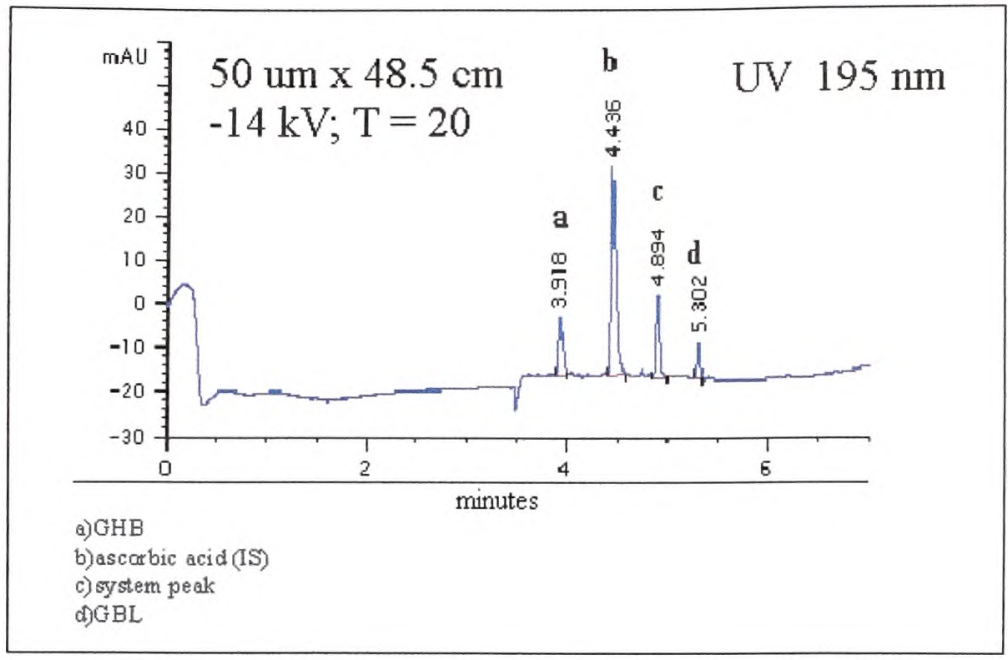

* Garcia, Lurie, Hulett, Almirall AAFS 2001 Proceedings. ${ }^{59,63}$

Figure 29: The separation of GHB (3.9 minutes) and GBL (5.3 minutes), using 25mM Phosphate/300mM CTAC pH 6.3, on a Hewlett Packard 3D CE. ${ }^{59,63}$

The internal standard, ascorbic acid, had a migration time of 4.4 minutes and the system peak appeared at 4.8 minutes. GHB gave a linear response from $0.63 \mathrm{mg} / \mathrm{ml}$ to $10.0 \mathrm{mg} / \mathrm{ml}$ (figure 30) and GBL was found to be linear from $0.99 \mathrm{mg} / \mathrm{ml}$ to $15.9 \mathrm{mg} / \mathrm{ml}$ (figure 31). The effects of temperature (figure 32) and voltage (figure 33) on the resolution and migration of the compounds were not significant. 


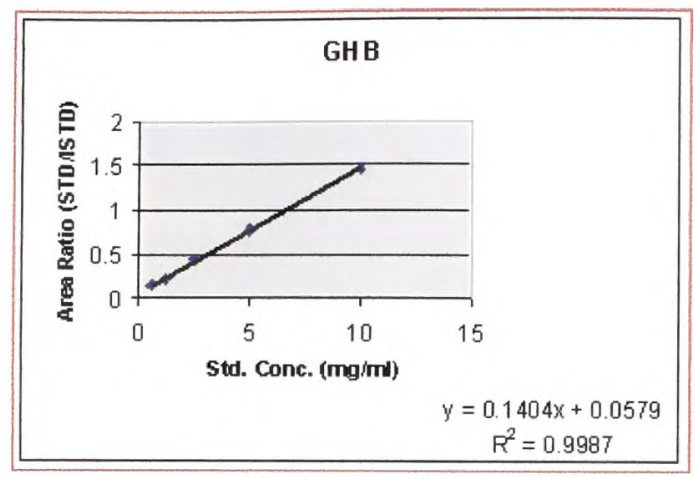

Standard 1: $0.63 \mathrm{mg} / \mathrm{ml}$

Standard 2: $1.25 \mathrm{mg} / \mathrm{ml}$

Standard 3:2.50 $\mathrm{mg} / \mathrm{ml}$

Standard 4: $5.00 \mathrm{mg} / \mathrm{ml}$

Standard 5: $10.00 \mathrm{mg} / \mathrm{ml}$

* Garcia, Lurie, Hulett, Almirall AAFS 2001 Proceedings. ${ }^{59,63}$

Figure 30: GHB Linearity Graph, using $25 \mathrm{mM}$ phosphate $/ 300 \mathrm{mM} \mathrm{CTAC} / \mathrm{pH} 6.3$, on a Hewlett Packard 3D CE. ${ }^{59,63}$ 


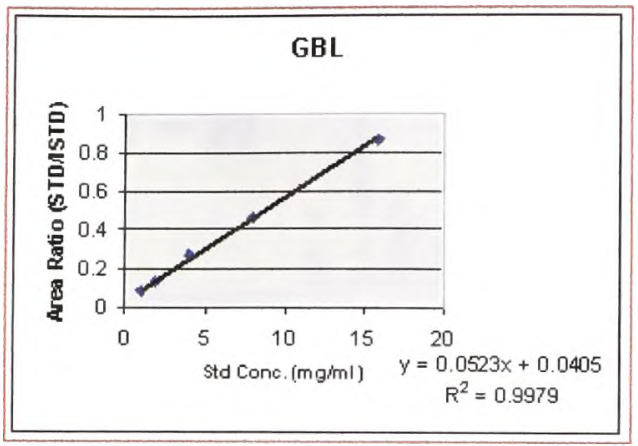

Standard 1: $0.99 \mathrm{mg} / \mathrm{ml}$ Standard 2: $1.99 \mathrm{mg} / \mathrm{ml}$ Standard 3: $3.98 \mathrm{mg} / \mathrm{ml}$ Standard 4: $7.95 \mathrm{mg} / \mathrm{ml}$ Standard 5: $15.90 \mathrm{mg} / \mathrm{ml}$

* Garcia, Lurie, Hulett, Almirall AAFS 2001 Proceedings. $^{59,63}$

Figure 31: GBL Linearity Graph, using 25mM phosphate $/ 300 \mathrm{mM} \mathrm{CTAC} / \mathrm{pH} 6.3$, on a Hewlett Packard 3D CE. ${ }^{59,63}$ 


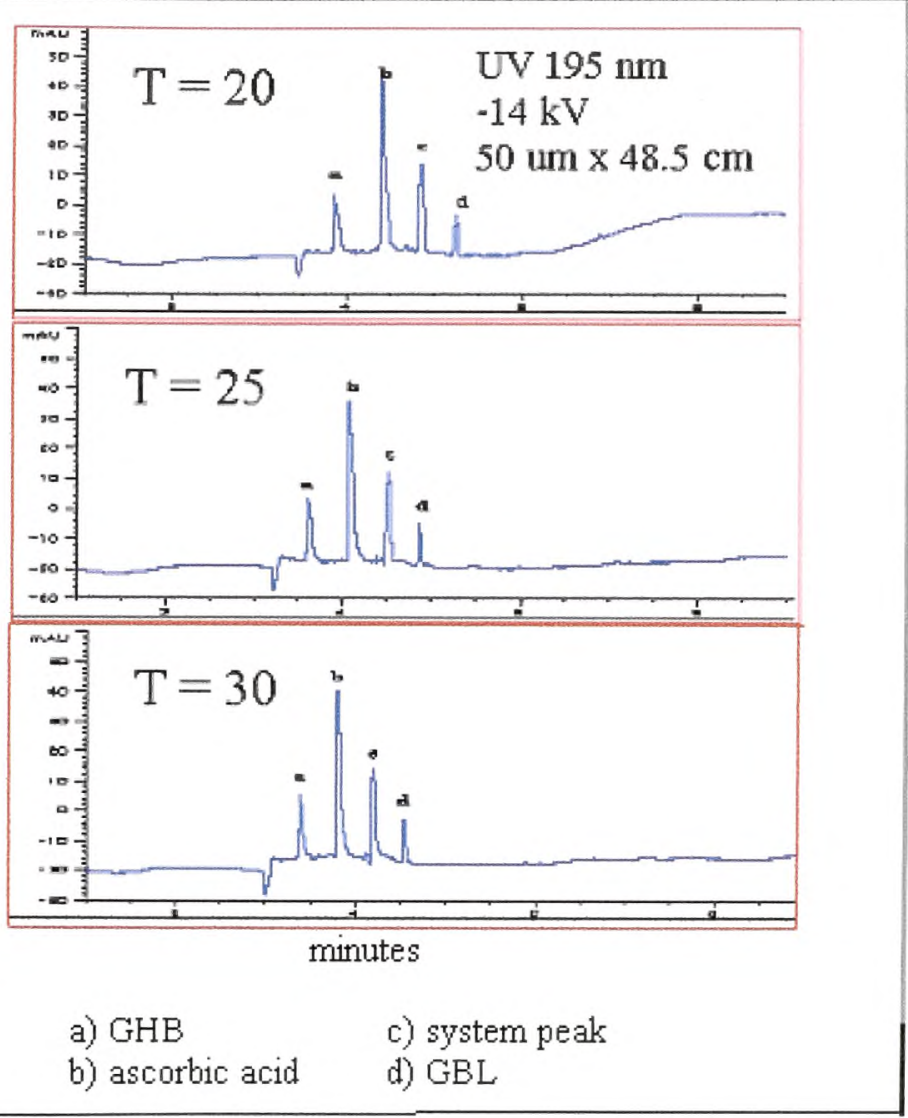

* Garcia, Lurie, Hulett, Almirall AAFS 2001 Proceedings. ${ }^{59,63}$

Figure 32: Effects of temperature on GHB analysis, using $25 \mathrm{mM}$ phosphate $/ 300 \mathrm{mM}$ CTAC/pH 6.3, on a Hewlett Packard 3D CE. ${ }^{59,63}$ 

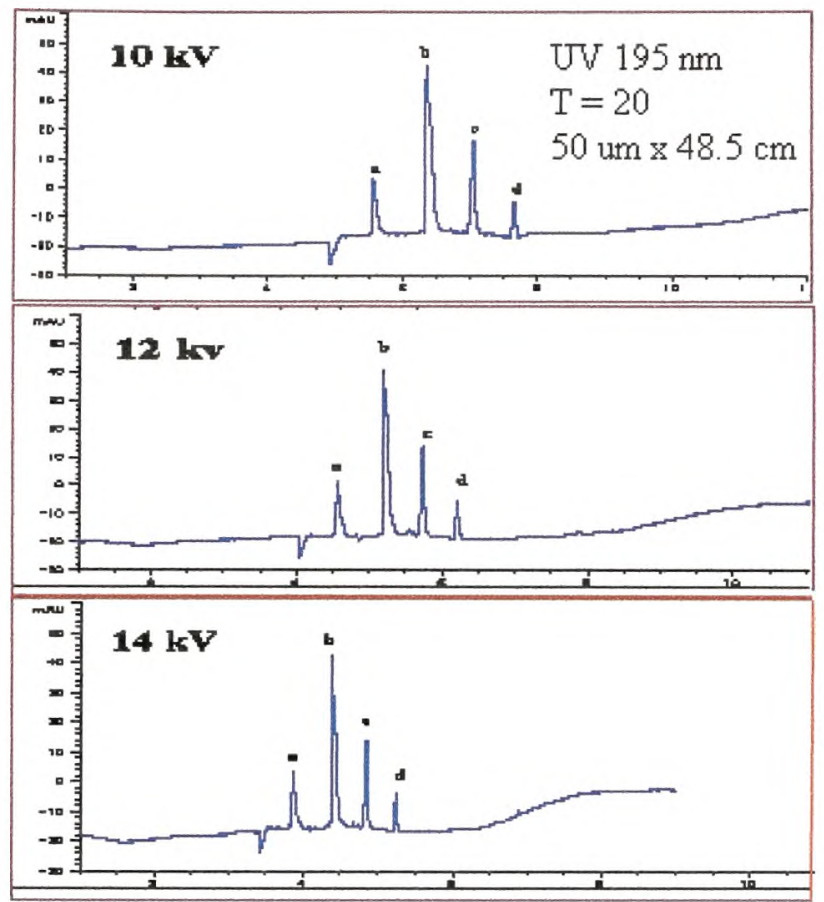

minutes
a) GHB
c) system peak
b) ascorbic acid
d) GBL

* Garcia, Lurie, Hulett, Almirall AAFS 2001 Proceedings. ${ }^{59,63}$

Figure 33: Effects of voltage on GHB analysis, using $25 \mathrm{mM}$ phosphate $/ 300 \mathrm{mM}$ CTAC/pH 6.3, on a Hewlett Packard 3D CE. ${ }^{59,63}$ 
Migration times for all compounds were reproducible with an RSD of less than $2 \%$ (table 1). The calculated bias from external calibration experiments with and without internal standards was less than $5 \%$.

\begin{tabular}{|c|c|c|c|}
\hline Corrpound & $\begin{array}{c}\text { Avg. Mgration } \\
\text { Time }\end{array}$ & $\begin{array}{c}\text { \%RSD } \\
\text { (n) }\end{array}$ & R \\
\hline GHB & 3.909 & 0.39 & Resolution \\
\hline GBL & 5.320 & 1.07 & * \\
\hline ascorbic acid OSTO) & 4441 & 0.44 & 12.0 \\
\hline
\end{tabular}

$25 \mathrm{mM}$ phosphate $/ 300 \mathrm{mM}$ CTAC/pH 6.3 on a Hewlett Packard 3D CE; 50 um x $48.5 \mathrm{~cm}$; UV $195 \mathrm{~nm}$

* Garcia, Lurie, Hulett, Almirall AAFS 2001 Proceedings. ${ }^{59,63}$

Table 1: Reproducibility data for GHB and GBL, using $25 \mathrm{mM}$ phosphate $/ 300 \mathrm{mM}$ CTAC/ pH 6.3 method. $^{59,63}$

\subsection{Phenylethylamines}

This author's results are part of two already published papers in which the author collaborated with primary authors Yuko Iwata and Ira Lurie ${ }^{56}$ : One, The use of highly sulfated cyclodextrin for the simultaneous chiral separation of amphetamine-type stimulants by capillary electrophoresis ${ }^{56}$, and second, Use of Dynamically Coated Capillaries for the Routine Analysis of Methamphetamine, Amphetamine, MDA, MDMA, MDEA, and Cocaine using Capillary Electrophoresis. ${ }^{19}$ 
The addition of HS- $\gamma-\mathrm{CD}$ to the Phosphate buffer of $\mathrm{pH} 7$, allowed for the chiral separation of 17 out of the 18 phenylethylamines (figure 34). Highly sulfated CDs contain sulfonic acid groups, which remain anionic for the entire $\mathrm{pH}$ range (figure 35 ). Not only do they have higher solubility than noncharged CDs, but they are chemically modified with negative groups, which act as countermigrating complexing agent. Using this mode, the optical isomers for norpseudoephedrine (cathine) were not distinguishable. However, this analysis would be suitable for the chiral determination and quantitation for 8 of the 9 racemic compounds.

$10 \mathrm{mM}$ HS-gamma-CD/50 mM phosphate/pH 7.0

UV $195 \mathrm{~nm} ; 52$ um x $34 \mathrm{~cm} ; 12 \mathrm{kV} ; \mathrm{T}=30$

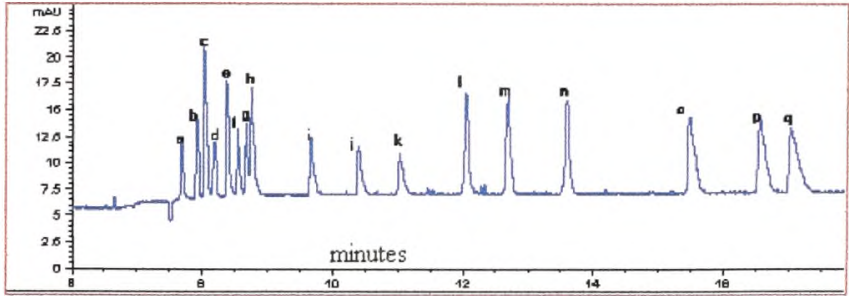
(a) d-norephedrine
(b) d-ephedrine
(j) 1-norephedrine
(c) d-amphetamine
(k) 1-amphetamine
(d) 1-pseudoephedrine
(1) $\mathrm{MDA}$
(e) d-pseudoephdrine
(m) MDMA
(f) 1-methamphetamine
(n) MDEA
(g) 1-ephedrine
(o) MDMA
(h) cathine
(p) MDEA
(i) d-methamphetarnine
(q) $\mathrm{MDA}$

* Garcia and Lurie. IAFS 1999 and AAFS 2000 Proceedings. ${ }^{60,61}$

Figure 34: Separation of 17 out of 18 phenylethylamines, using HS- $\gamma$-CD/Phosphate buffer at $\mathrm{pH} 7.0$, on a Hewlett Packard 3D CE. ${ }^{60,61}$ 


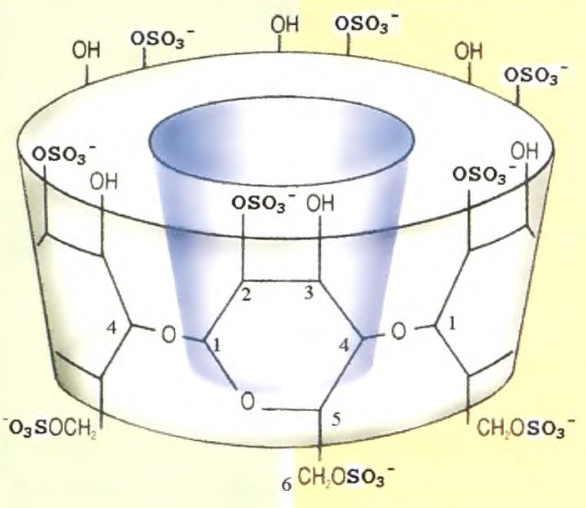

* Figure obtained from DEA Senior Forensic Chemist Ira Lurie.

Figure 35: Diagram of HS- $\gamma$-CD.

The simultaneous quantitation and chiral determination of methamphetamine and MDMA was attempted using this above method. On figure 36, the effects of temperature, at $12 \mathrm{kV}$, on the chiral determination of ephedrine, pseudoephedrine, and methamphetamine are demonstrated. Separation at $20^{\circ} \mathrm{C}$ caused a loss in resolution between d-pseudoephedrine and 1-methamphetamine At a temperature of $35^{\circ} \mathrm{C}$, there was a loss in resolution between d-ephedrine and 1-pseudoephedrine. 

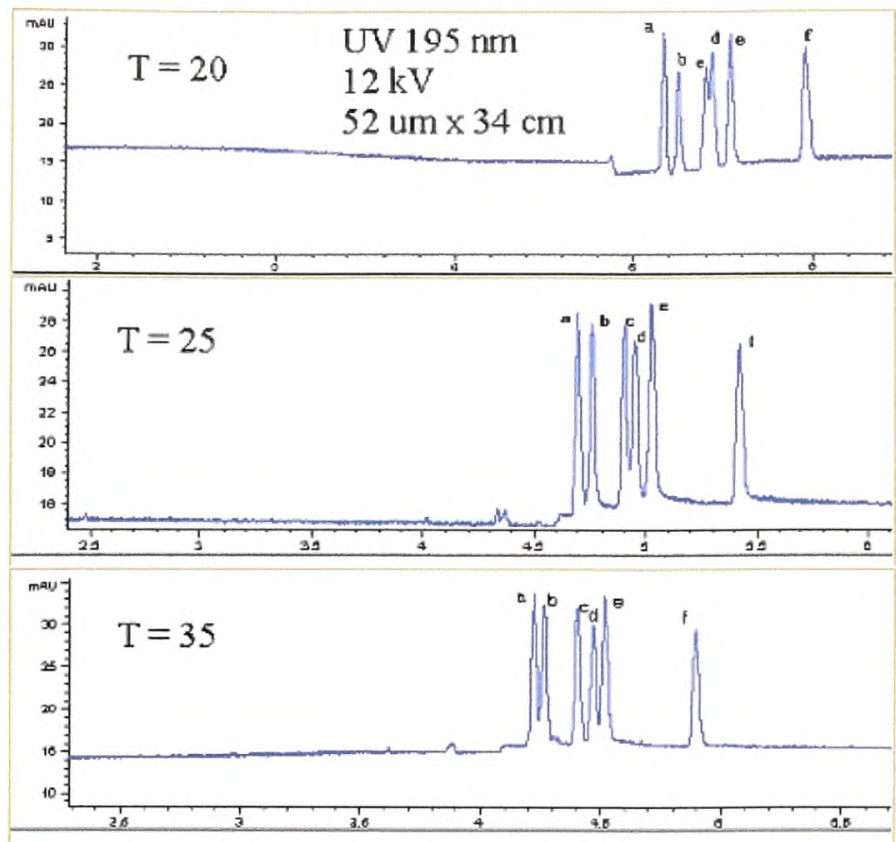
(a) d-ephectine
(b) 1-pseudoephedtine
(c) d-pseudoephedrine
(d) - methamphetamine
(c) 1-ephedtire
(f) d-methamphetamine

minutes

* Garcia and Lurie. IAFS 1999 Proceedings. ${ }^{60}$

Figure 36: Effects of Temperature on methamphetamine, using 10mM HS- $\gamma-\mathrm{CD} / 50 \mathrm{mM}$ phosphate/pH 7.0, on a Hewlett Packard 3D CE. ${ }^{60}$

Optimized method conditions were found to be at $30^{\circ} \mathrm{C}$ and $12 \mathrm{kV}$. The spectra of the quantitation of 2 methamphetamine samples (at $2 \%$ and $31.2 \%$ respectively) were 
performed, using n-butylamphetamine as the internal standard and are shown in figure 37 and 38 .

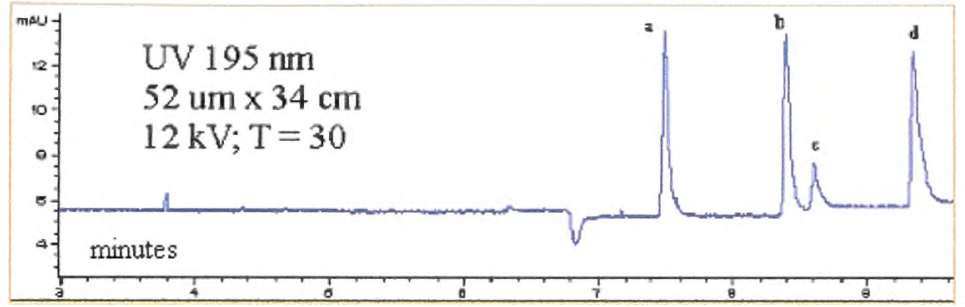

(a) dim ethyl sulfone from sample

(b) a-n-butylamphetamine

(c) dmetham phetamine from sam ple (2.0\%)

(d) I-n-butylampheteanine

* Garcia and Lurie. IAFS 1999 Proceedings. ${ }^{60}$

Figure 37: Methamphetamine case sample, with $2.0 \%$ methamphetamine, using $10 \mathrm{mM}$ HS- $\gamma-\mathrm{CD} / 50 \mathrm{mM}$ phosphate/pH 7.0 on a Hewlett Packard 3D CE. ${ }^{60}$ 


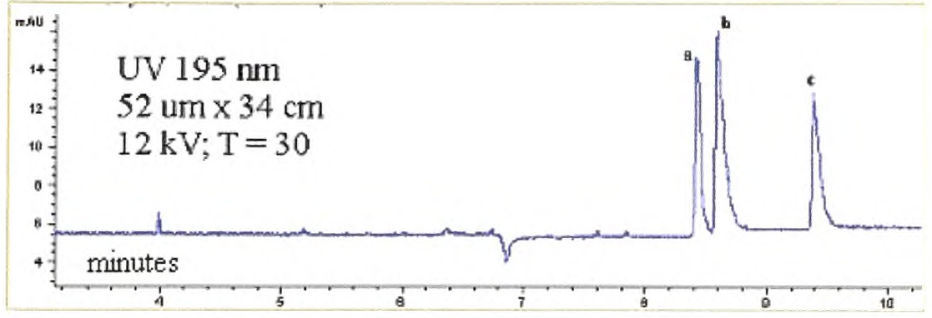

(a) d-n-butyi an phetamine

(b) I-methamphetanine from sample (31.2\%)

(c) 1-n-butylem phetamine

* Garcia and Lurie. IAFS 1999 Proceedings. ${ }^{60}$

Figure 38: Methamphetamine case sample, with $31.2 \%$ methamphetamine, using $10 \mathrm{mM}$ HS- $\gamma-\mathrm{CD} / 50 \mathrm{mM}$ phosphate/pH 7.0 on a Hewlett Packard 3D CE. ${ }^{60}$

Methamphetamine had a linear response from $0.03 \mathrm{mg} / \mathrm{ml}$ to $1.0 \mathrm{mg} / \mathrm{ml}$, with a correlation coefficient of 0.9997 (figure 39). The resolution between $\mathrm{d}$ and 1 methamphetamine was found to be 20.7. Migration times for methamphetamine, amphetamine, ephedrine, pseudoephedrine, and MDMA were reproducible with an RSD of less than $1.5 \%$ (table 2$)$. 


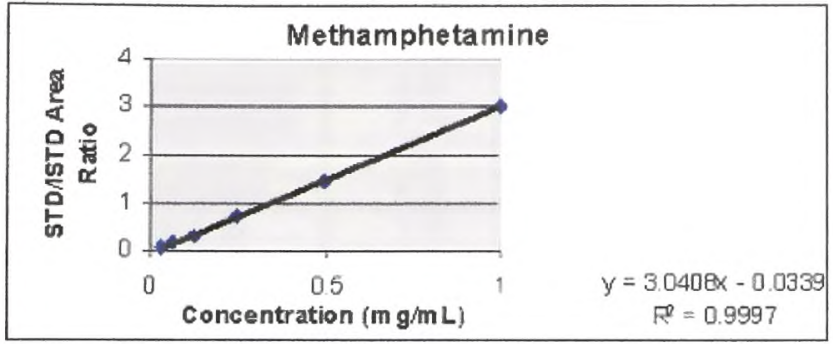

Standard 1: $0.03 \mathrm{mg} / \mathrm{ml}$

Standard 2: $0.06 \mathrm{mg} / \mathrm{ml}$

Standard 3: $0.13 \mathrm{mg} / \mathrm{ml}$

Standard 4: $0.25 \mathrm{mg} / \mathrm{ml}$

Standard 5: $0.50 \mathrm{mg} / \mathrm{ml}$

Standard 6: $1.00 \mathrm{mg} / \mathrm{ml}$

* Garcia and Lurie. IAFS 2001 Proceedings. ${ }^{60}$

Figure 39: Linearity for methamphetamine, using $10 \mathrm{mM} \mathrm{HS}-\gamma-\mathrm{CD} / 50 \mathrm{mM}$ phosphate/pH 7.0 on a Hewlett Packard 3D CE. ${ }^{60}$ 


\begin{tabular}{|c|c|c|c|}
\hline Compound & $\begin{array}{l}\text { Avg. Migration } \\
\text { Time }\end{array}$ & $\begin{array}{c}\% \mathrm{RSD} \\
n=5\end{array}$ & $\mathrm{R}$ \\
\hline d-methamphetamine & 8.65 & 0.95 & \multirow[t]{2}{*}{20.7} \\
\hline I-methamphetamine & 7.72 & 0.84 & \\
\hline d-amphetamine & 7.29 & 0.80 & \multirow[t]{2}{*}{46.8} \\
\hline I-amphetamine & 9.74 & 1.06 & \\
\hline d-ephedrine & 7.19 & 0.79 & \multirow[t]{2}{*}{16.6} \\
\hline I-ephedrine & 7.82 & 0.85 & \\
\hline d-pseudoephednine & 7.58 & 0.82 & \multirow[t]{2}{*}{4.1} \\
\hline I-pseudoephedrine & 7.41 & 0.80 & \\
\hline MDMA & 11.04 & 1.24 & \multirow[t]{2}{*}{26.2} \\
\hline MDMA & 13.16 & 1.39 & \\
\hline
\end{tabular}

Reproducibility data for methamphetamine, using $10 \mathrm{mM}$ HS-gamma-CD/50mM phosphate/pH 7.0; $52 \mathrm{um} \times 34 \mathrm{~cm} ; 12 \mathrm{kV} ; \mathrm{T}=30$

* Garcia and Lurie. IAFS 1999 Proceedings. ${ }^{60}$

Table 2: Reproducibility data for methamphetamine, using $10 \mathrm{mM}$ HS- $\gamma-\mathrm{CD} / 50 \mathrm{mM}$ phosphate/pH 7.0, on a Hewlett Packard 3D CE. ${ }^{60}$

Using this same background electrolyte system of $10 \mathrm{mM} \mathrm{HS}-\gamma-\mathrm{CD} / 50 \mathrm{mM}$ Phosphate buffer pH 7.0, MDMA was quantitated. Figure 40 shows the effects of temperature on the separation of MDMA, MDA, and MDEA isomers, while figure 41 demonstrates the effects of voltage. Optimized method conditions were found to be at $30^{\circ} \mathrm{C}$ and $12 \mathrm{kV}$. 


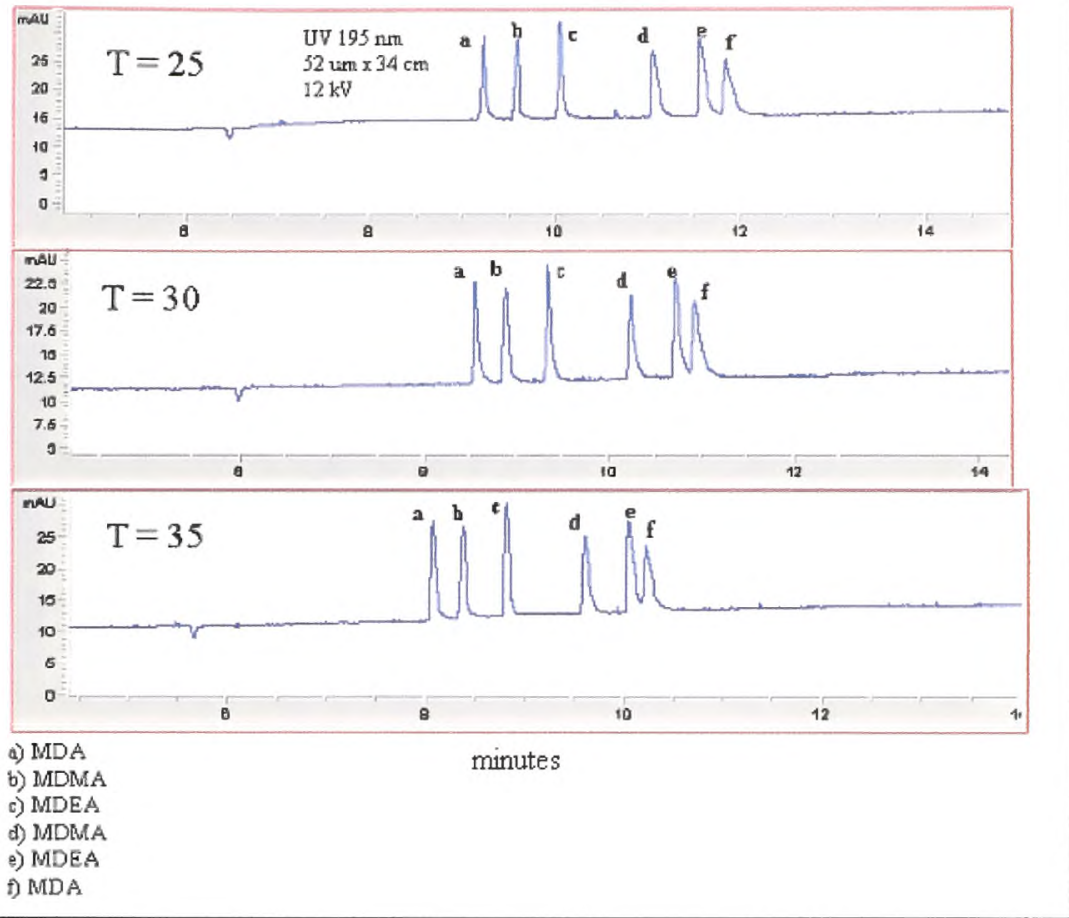

* Garcia and Lurie. AAFS 2000 Proceedings. ${ }^{61}$

Figure 40: Effects of Temperature on MDMA analysis, using 10mM HS- $\gamma-\mathrm{CD} / 50 \mathrm{mM}$ phosphate/pH 7.0, on a Hewlett Packard 3D CE. ${ }^{61}$ 


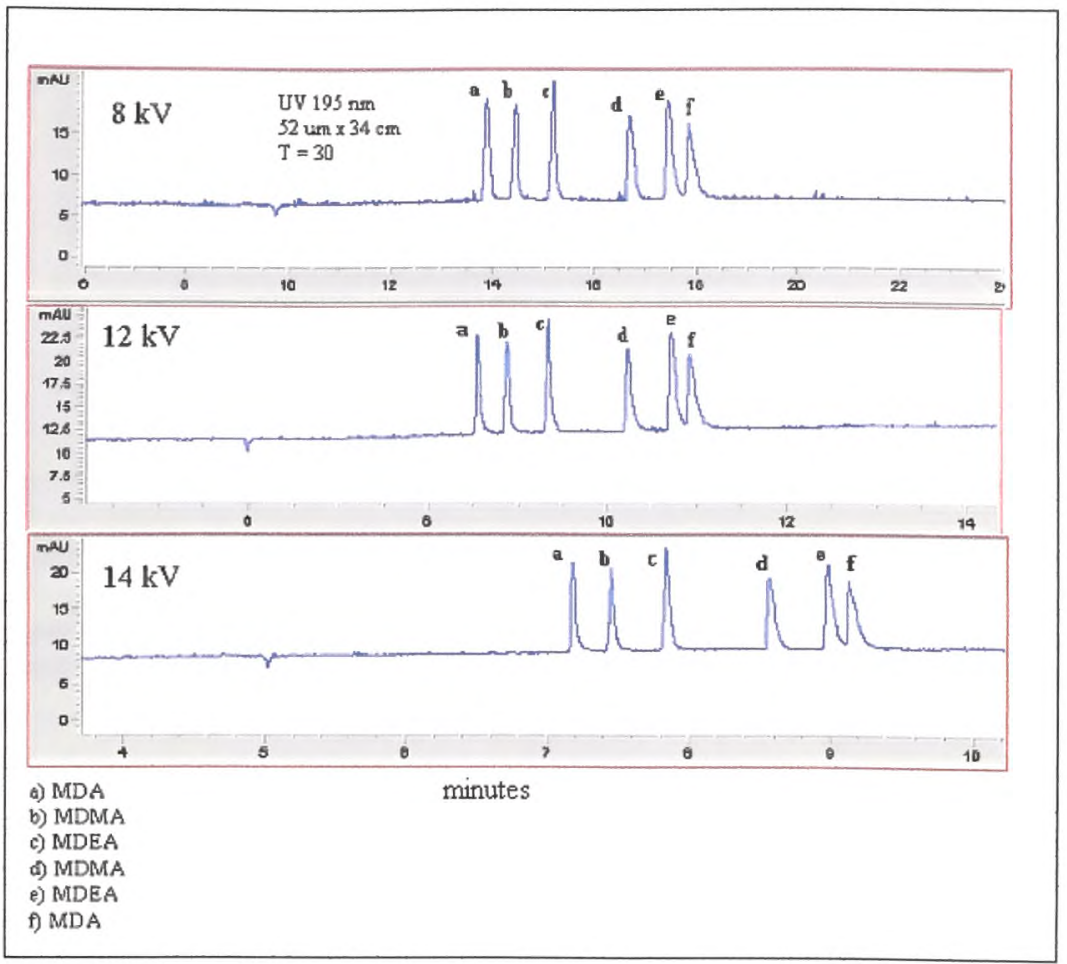

* Garcia and Lurie. AAFS 2000 Proceedings. ${ }^{61}$

Figure 41: Effects of voltage on MDMA analysis, using 10mM HS- $\gamma-\mathrm{CD} / 50 \mathrm{mM}$ phosphate/pH 7.0, on a Hewlett Packard 3D CE. ${ }^{61}$

The spectra of the quantitation of 2 MDMA samples were performed, using nbutylamphetamine as the internal standard and are shown in figure 42 and 43. 


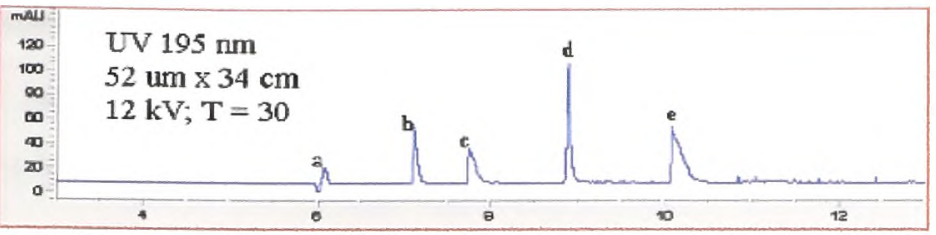

a) C affeine (froen sample)

minutes

b) N-butyiamphetamine (ISTD)

c) N-butylam phetarn ine (ISTD)

d) MDMA

e) MDMA

* Garcia and Lurie. AAFS 2000 Proceedings. ${ }^{61}$

Figure 42: MDMA illicit sample, using 10mM HS-- $\gamma-\mathrm{CD} / 50 \mathrm{mM}$ phosphate/pH 7.0, on a Hewlett Packard 3D CE. ${ }^{61}$

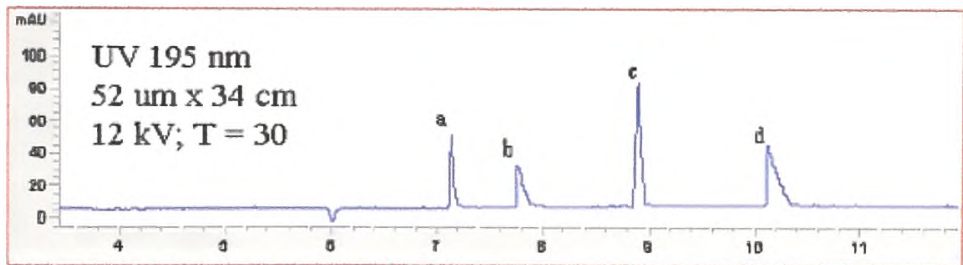

a) N-butylamphetam ine (ISTD)

minutes

b) N-butyl am phetamine (ISTD)

c) MDMA

d) MDMA

* Garcia and Lurie. AAFS 2000 Proceedings. ${ }^{61}$

Figure 43: MDMA illicit sample, using 10mM HS-- $\gamma-\mathrm{CD} / 50 \mathrm{mM}$ phosphate/pH 7.0, on a Hewlett Packard 3D CE. ${ }^{61}$ 
MDMA had a linear response from $0.02 \mathrm{mg} / \mathrm{ml}$ to $1.0 \mathrm{mg} / \mathrm{ml}$, with a correlation coefficient of 0.999 (figure 44). The resolution between d and I MDMA was found to be 26.2. Migration times for MDMA, MDA, and MDEA isomers were reproducible with an RSD of less than $1.5 \%$ (table 3 ).

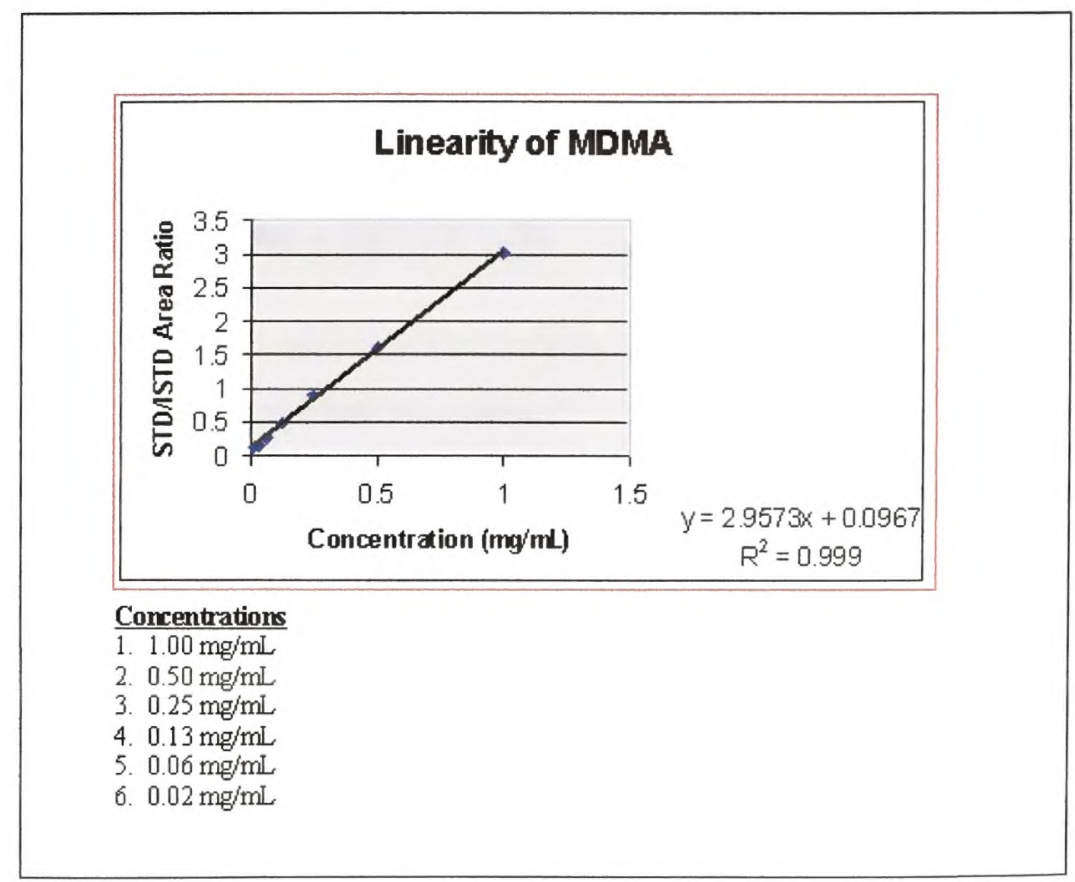

* Garcia and Lurie. AAFS 2000 Proceedings. ${ }^{61}$

Figure 44: Linearity of MDMA, using $10 \mathrm{mM} \mathrm{HS}--\gamma-\mathrm{CD} / 50 \mathrm{mM}$ phosphate $/ \mathrm{pH} 7.0$, on a Hewlett Packard 3D CE. ${ }^{61}$ 


\begin{tabular}{|c|c|c|c|}
\hline Compound & $\begin{array}{l}\text { Avg. Migration } \\
\text { Time }\end{array}$ & $\begin{array}{l}\text { \%RSD } \\
(n=5)\end{array}$ & $\begin{array}{c}\mathbf{R} \\
\text { Resolution }\end{array}$ \\
\hline MDMA & 11.04 & 1.24 & 26.2 \\
\hline MDMA & 13.16 & 1.39 & \\
\hline MDEA & 11.75 & 1.33 & 27.7 \\
\hline MDEA & 13.97 & 1.41 & \\
\hline MDA & 10.54 & 1.17 & 46.7 \\
\hline MDA & 14.32 & 1.4 & \\
\hline d-methamphetamine & 8.65 & 0.95 & 20.7 \\
\hline I-metham phetamine & 7.72 & 0.84 & \\
\hline d-amphetamine & 7.29 & 0.8 & 46.8 \\
\hline I-amphetamine & 9.74 & 1.06 & \\
\hline \multicolumn{4}{|c|}{$\begin{array}{l}\text { Reproducibility data for MDMA, using } 10 \mathrm{mM} \\
\text { HS-gamma-CD/50mM phosphate/pH 7.0; } \\
52 \mathrm{um} \text { x } 34 \mathrm{~cm} ; 12 \mathrm{kV} ; \mathrm{T}=30\end{array}$} \\
\hline
\end{tabular}

* Garcia and Lurie. AAFS 2000 Proceedings. ${ }^{61}$

Table 3: Reproducibility data for MDMA illicit samples, using $10 \mathrm{mM}$ HS-- $\gamma-\mathrm{CD} / 50 \mathrm{mM}$ phosphate/pH 7.0, on a Hewlett Packard 3D CE. ${ }^{61}$

The addition of $10 \mathrm{mM}$ HS- $\gamma$-CD to $50 \mathrm{mM}$ Phosphate buffer of $\mathrm{pH} 2.6$, allowed for the chiral separation of all 18 phenylethylamines (figure 45). This separation is the optimum chiral separation with complete separation of all 18 compounds. Analysis time was within 32 minutes, with an applied voltage of $-12 \mathrm{kV}$ and a temperature of $15^{\circ} \mathrm{C}$. "...the large resolution between d-methamphetamine and other solutes allows for methamphetamine sample overload...". 56 


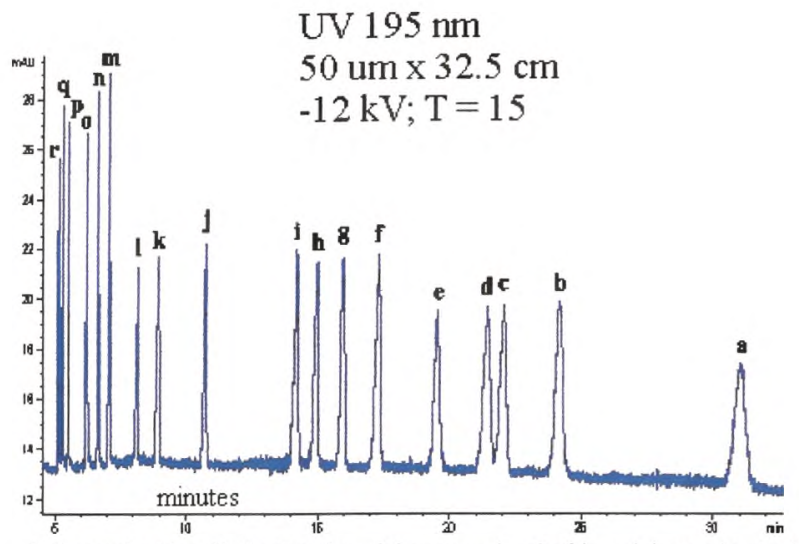

a) d-norephedrine b) d-ephedrine c) 1-norpseudoephedrine d) 1-pseudoephedrine

e) 1-amphetamine f) d-pseudoephedrine gl 1-methamphetamine h) 1-ephedrine

i) a norpseudoephedrine j) d-methamphetamine k) 1-norephedrine

1) (t) amphetamine m) MDA n) MDMA o) MDEA p) MDMA q) MDEA r) MDA

* Electropherogram obtained from Yuko Iwata, Garcia, et. al. ${ }^{56}$

Figure 45: Separation of all 18 phenylethylamines, using $10 \mathrm{mM}$ HS- $\gamma-\mathrm{CD} / 50 \mathrm{mM}$ phosphate/pH 2.5, on a Hewlett Packard 3D CE. ${ }^{56}$

Dynamic coating provides reproducible and rugged separations. While generally low $\mathrm{pH}$ background electrolytes create low EOF, with dynamic coating low $\mathrm{pH}$ generates high EOF. In figure 46, the dynamic coating separation of methamphetamine, amphetamine, MDMA, MDA, and MDEA is demonstrated. This system used the CE lixir buffer system $\mathrm{pH} 2.5$ at $15^{\circ} \mathrm{C}$ with an applied voltage of $10 \mathrm{kV}$, as previously reported by 
Lurie in $2001 .{ }^{19}$ At a wavelength of $200 \mathrm{~nm}$, there is excellent resolution between the aforementioned solutes. An overlay of 5 runs showed migration times to have an RSD value of 0.04 , while area ratios had an RSD range of 0.095-0.29. ${ }^{19}$

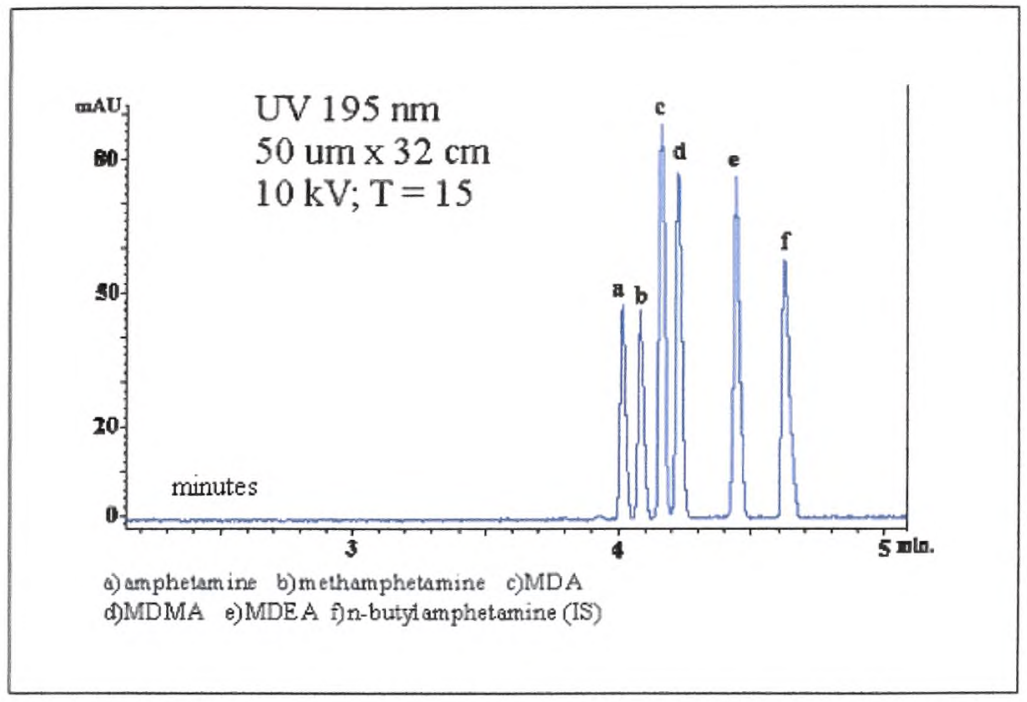

* Electropherogram obtained from DEA Senior Forensic Chemist Ira Lurie. ${ }^{19}$

Figure 46: Dynamic Coating for methamphetamine and related compounds, using CElixir pH 2.5 on a Hewlett Packard 3D CE. ${ }^{19}$

Chiral analysis can also be performed very efficiently with dynamic coating, as was reported by Lurie in 2001. ${ }^{19}$ Figure 47, shows the chiral determination of methamphetamine, using dynamic coating. Quantitation and chiral determination of 
methamphetamine can be performed simultaneously, using the CE lixir buffer kit with $50 \mathrm{mM}$ of HP-B-CD. ${ }^{19}$

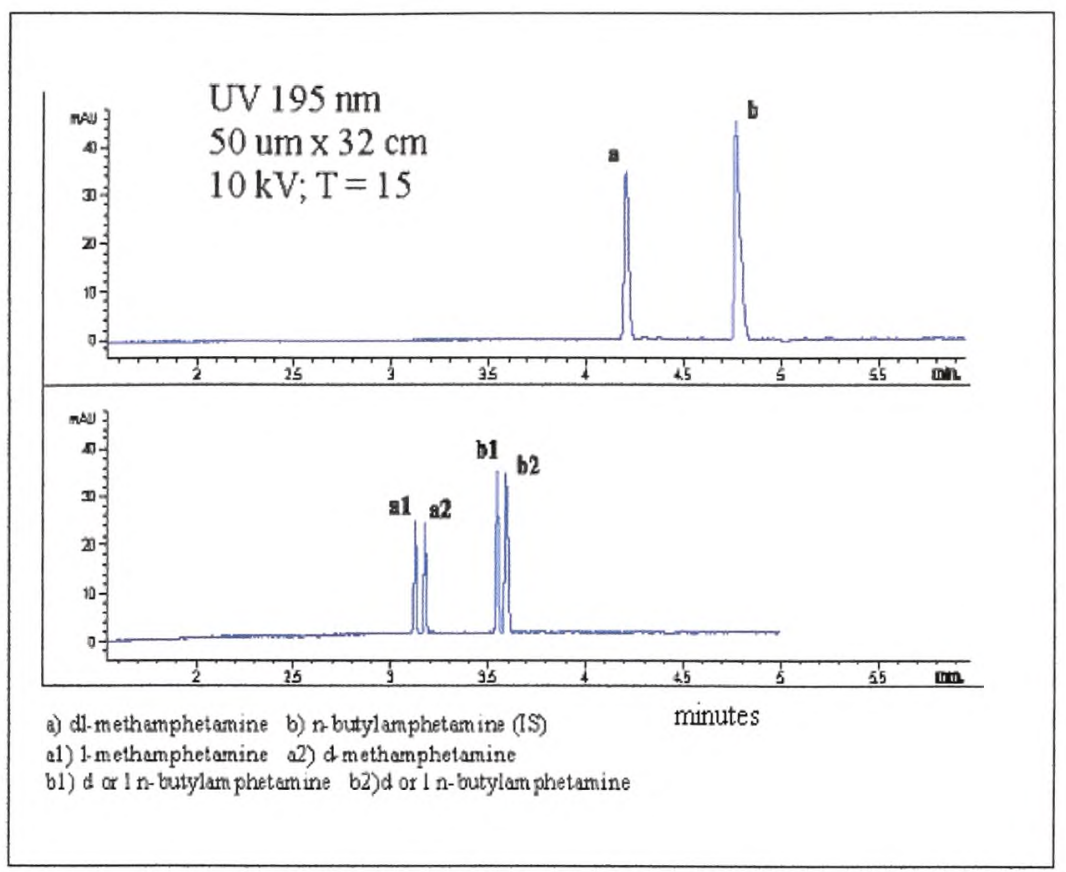

* Figure obtained from DEA Senior Forensic Chemist Ira Lurie. ${ }^{19}$

Figure 47: Quantitation and Chiral determination of methamphetamine, using CElixir $\mathrm{pH}$ $2.5 / 50 \mathrm{mM}$ HP- $\beta-\mathrm{CD}$ on a Hewlett Packard 3D CE. ${ }^{19}$

\subsection{Psilocybin}

The Cyanescens mushroom was found to contain psilocybin as the major hallucinogenic constituent. Some of the mushrooms were found to contain detectable amounts of psilocin. 
UV $254 \mathrm{~nm}$

$25 \mathrm{um} \times 57 \mathrm{~cm}$

$8 \mathrm{kV} ; \mathrm{T}=25$

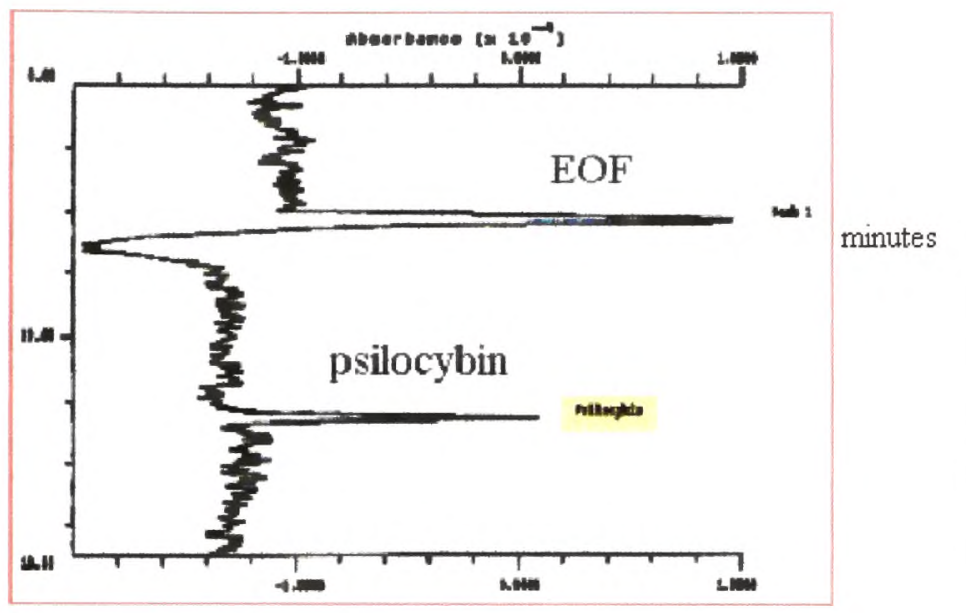

* Garcia, Gross and Almirall. IAFS 1999 Proceedings. ${ }^{62}$

Figure 48: Analysis of psilocybin, using $10 \mathrm{mM}$ borate/10mM phosphate/pH 11.5, on a Beckman P/ACE System 5000 CE. ${ }^{62}$

Using CZE, psilocybin was found to migrate at 11.3 minutes (see figure 48) and at 9.9 with MECC (see figures 49, 50, and 51). 
UV $210 \mathrm{~nm}$

$50 \mathrm{um} \times 50 \mathrm{~cm}$

$12 \mathrm{kV} ; \mathrm{T}=25$

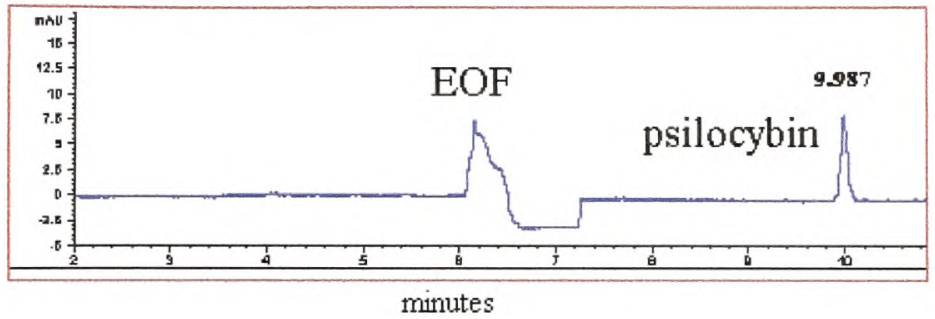

100 ppm psilocybin standard

* Garcia, Gross and Almirall. IAFS 1999 Proceedings. ${ }^{62}$

Figure 49: Psilocybin Standard (100 ppm), using $85 \mathrm{mM}$ SDS $/ 8.5 \mathrm{mM}$ phosphate $/ 8.5 \mathrm{mM}$ borate $/ 15 \% \mathrm{ACN} / \mathrm{pH} 8.5$, on a Hewlett Packard 3D CE. ${ }^{62}$ 
UV $210 \mathrm{~nm}$

$50 \mathrm{um} \times 50 \mathrm{~cm}$

$12 \mathrm{kV} ; \mathrm{T}=25$

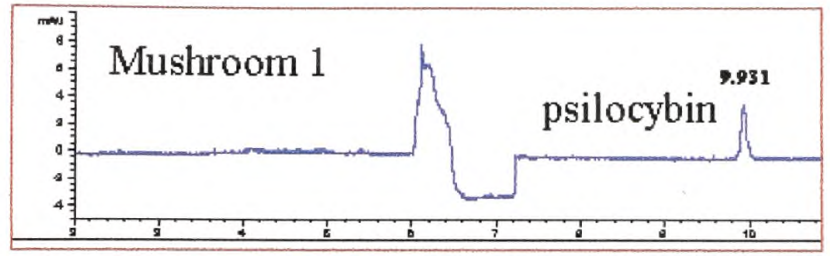

minutes

* Garcia, Gross and Almirall. IAFS 1999 Proceedings. ${ }^{62}$

Figure 50: Mushroom 1 using $85 \mathrm{mM}$ SDS $/ 8.5 \mathrm{mM}$ phosphate $/ 8.5 \mathrm{mM}$ borate $/ 15 \%$ $\mathrm{ACN} / \mathrm{pH} 8.5$, on a Hewlett Packard 3D CE. ${ }^{62}$

UV $210 \mathrm{~nm}$

$50 \mathrm{um} \times 50 \mathrm{~cm}$

$12 \mathrm{kV} ; \mathrm{T}=25$

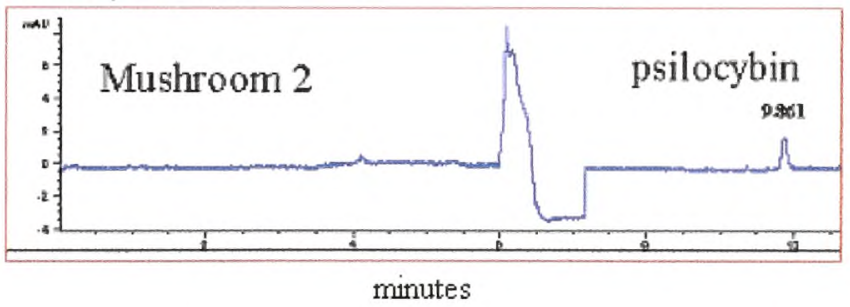

* Garcia, Gross and Almirall. IAFS 1999 Proceedings. ${ }^{62}$

Figure 51: Mushroom 2 using $85 \mathrm{mM}$ SDS $/ 8.5 \mathrm{mM}$ phosphate $/ 8.5 \mathrm{mM}$ borate $/ 15 \%$ $\mathrm{ACN} / \mathrm{pH} 8.5$, on a Hewlett Packard 3D CE. ${ }^{62}$ 
The calibration curves for psilocybin based on peak area was linear in the concentration range of 0.01 to $0.05 \mathrm{mg} / \mathrm{mL}$ using CZE, and 0.006 to $0.10 \mathrm{mg} / \mathrm{mL}$ using MECC (figures 52 and 53). Since no large amounts of psilocin was found in the mushrooms, this compound was not quantitated. Mushroom 4 was found to contain detectable amounts psilocin (see figure 54). CZE and MECC results were compared to HPLC data.

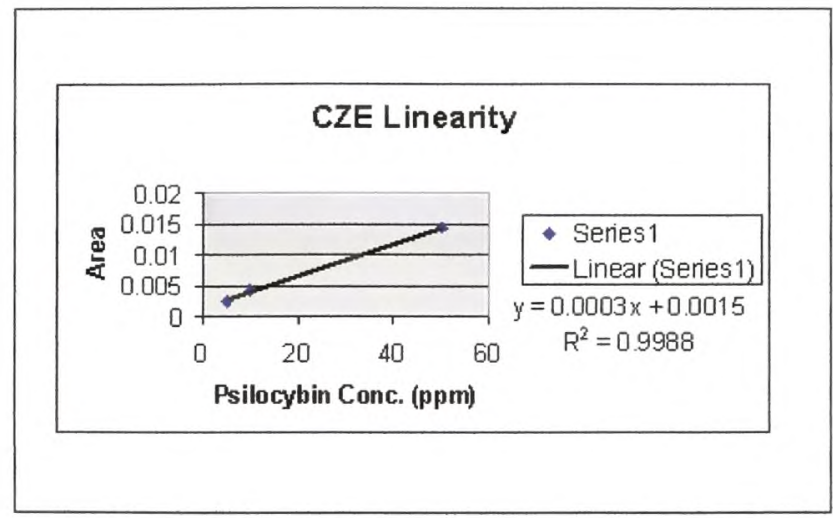

* Garcia, Gross and Almirall. IAFS 1999 Proceedings. ${ }^{62}$

Figure 52: CZE Linearity of psilocybin, using $10 \mathrm{mM}$ borate $/ 10 \mathrm{mM}$ phosphate/pH 11.5 , on a Beckman P/ACE System $5000 \mathrm{CE} .^{62}$ 


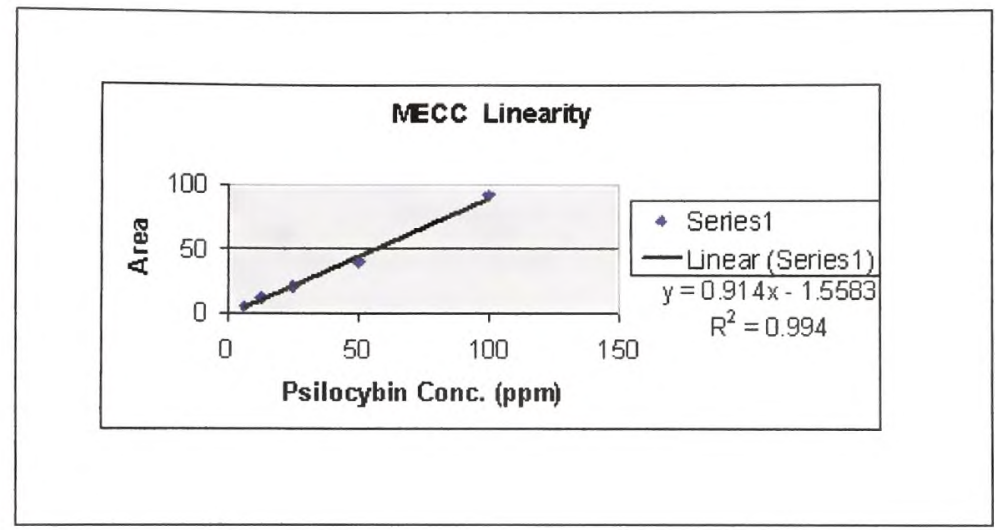

* Garcia, Gross and Almirall. IAFS 1999 Proceedings. $^{62}$

Figure 53: MECC Linearity of psilocybin, using $85 \mathrm{mM}$ SDS $/ 8.5 \mathrm{mM}$ phosphate $/ 8.5 \mathrm{mM}$ borate/15\% ACN/pH 8.5, on a Hewlett Packard 3D CE. ${ }^{62}$ 
UV $254 \mathrm{~nm}$

$25 \mathrm{um} \times 57 \mathrm{~cm}$

$8 \mathrm{kV} ; \mathrm{T}=25$

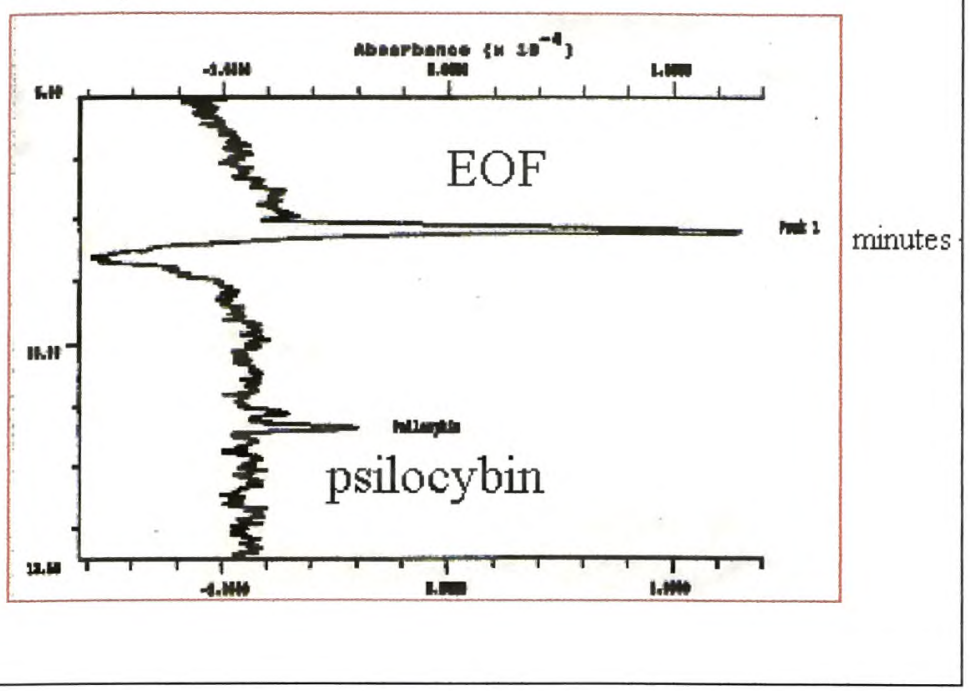

* Garcia, Gross and Almirall. IAFS 1999 Proceedings. ${ }^{62}$

Figure 54: Analysis of Mushroom 4, using $10 \mathrm{mM}$ borate/10mM phosphate/pH 11.5, on a Beckman P/ACE System 5000 CE. $^{62}$

Mushroom 2 was found to contain the highest concentration of psilocybin per unit weight, with $0.05 \%$ psilocybin of $242.8 \mathrm{mg}$ of mushroom mass. Mushroom 3 was found to contain the least amount of psilocybin with $0.008 \%$ of $1486.8 \mathrm{mg}$ of mushroom. Mushroom 4 was found to contain detectable amounts of psilocin. 


\subsection{Opium}

In 1995, Bjornsdottir and Hansen reported a CE method for the analysis of opium, which entailed the use of several different cyclodextrins. Bjornsdottir investigated the separation of morphine, codeine, papaverine, thebaine, and noscapine, using 30mM DMB-CD, 20mM TM-B-CD, 20mM HP-B-CD, 30mM gamma-CD, and 5mM SB-B-CD. ${ }^{64}$ Other methods using SDS or CTAB as the surfactant have also been reported. However, these methods only looked at the separation of morphine, codeine, and noscapine.

This author's results are part of an already published paper in which the author collaborated with the primary author Ira Lurie: Use of Dynamically Coated Capillaries with Added Cyclodextrins for the Analysis of Opium using Capillary Electrophoresis. ${ }^{58}$ In this study, the addition of DM-B-CD and HP-B-CD to the CElixir Reagent $\mathrm{B}$ of $\mathrm{pH} 2.5$ was explored. Adding either of these cyclodextrins affected the migration times of opium alkaloids. As previously reported by Lurie et al in $2002^{58}$, the relative migration time of thebaine relative to noscapine and morphine relative to papaverine is significantly greater for HP-B-CD than with DM-B-CD. Thus, optimal method conditions consisted of a mixture of these two cyclodextrins, $25 \mathrm{mM}$ of HP-B-CD and $75 \mathrm{mM}$ of DM-B-CD, respectively.

Excellent separation was obtained for 5 major opium alkaloids, morphine, papaverine, codeine, noscapine, and thebaine, respectively (figure 55). Tetracaine hydrochloride was used as the internal standard. However, run-to-run peak area precision was poor. Samples were dissolved in a small amount of methanol, to reduce the viscosity of the injection solvent ( $3.75 \mathrm{mM}$ phosphate buffer $\mathrm{pH} 3.2$ with now $10 \%$ methanol). 
Samples were also sonicated for 30 minutes - 1 hour, due to noscapine and papaverine having limited solubility.

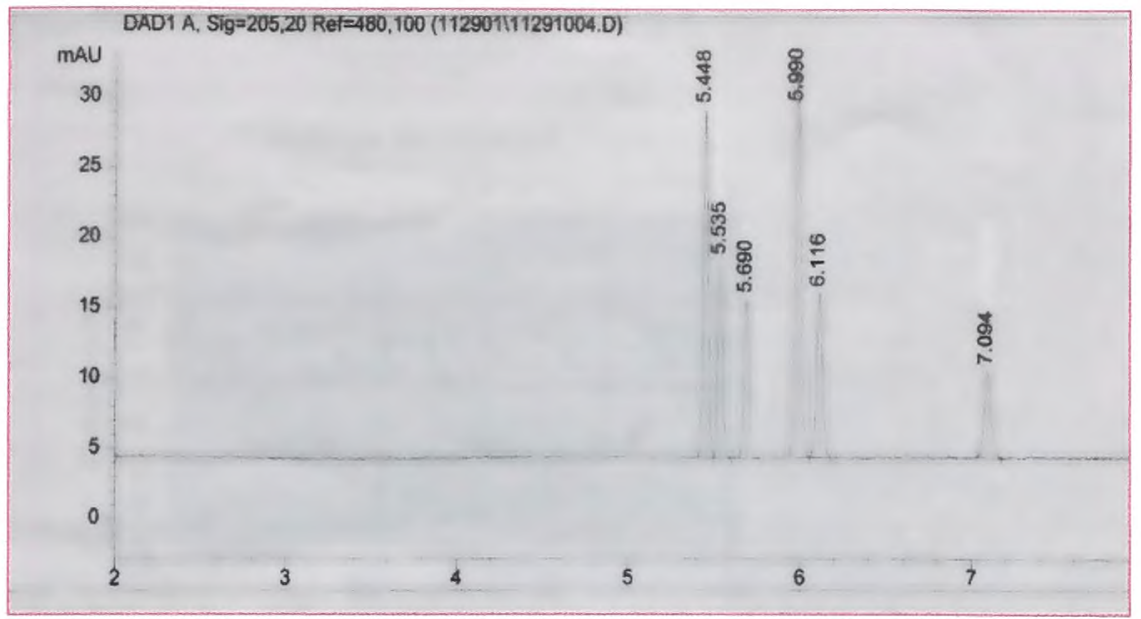

Mixture of morphine (5.4 min), papaverine (5.5 min), codeine (5.6 min), noscapine (5.9 $\mathrm{min})$, thebaine $(6.1 \mathrm{~min})$, and tetracaine $(7.0 \mathrm{~min})$ as the internal standard.

Figure 55: Dynamic Coating for Opium, using 50mM of DM-B-CD/CElixir pH 2.5.

The same opium sample (mixture of morphine, papaverine, codeine, noscapine, thebaine, and tetracaine) was analyzed using 100mM DM-B-CD/CElixir pH 2.5 and using 25mM DM-B-CD with CElixir $\mathrm{pH} 2.5$. There was a loss of resolution between noscapine and thebaine at $100 \mathrm{mM}$ DM-B-CD, while a loss of resolution occurred between morphine/ papaverine and codeine/noscapine at the $25 \mathrm{mM}$ DM-B-CD. ${ }^{58}$ 


\subsection{Benzodiazepines}

The $50 \mathrm{mM}$ Phosphate ( $\mathrm{pH} 2.5$ ) running buffer was chosen due to the pKa's of diazepam and flunitrazepam being 3.3 and 1.8. Diazepam showed a much larger absorbance at $210 \mathrm{~nm}$ than flunitrazepam.

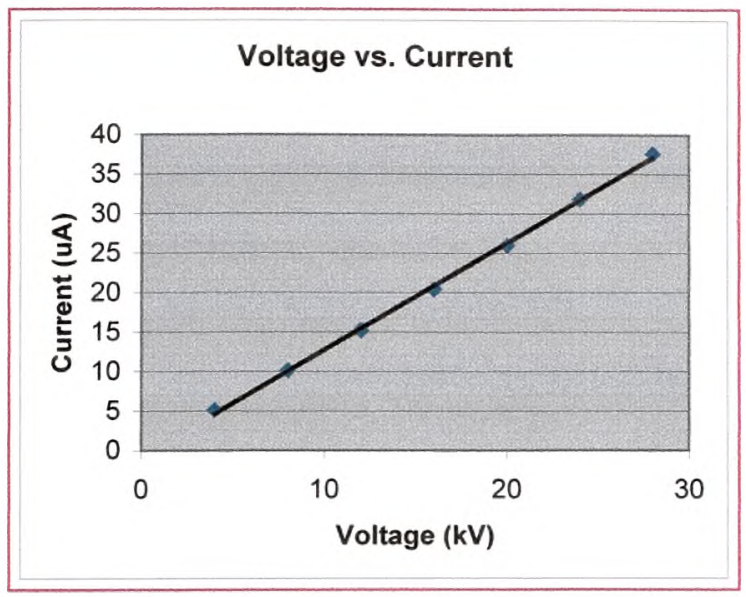

Figure 56: Ohm's Law Plot showing excessive current and possible joule heating over 27 $\mathrm{kV}$.

An Ohms's law plot performed with a $50 \mathrm{mM}$ phosphate buffer $\mathrm{pH} 2.5$ and a $25^{\circ} \mathrm{C}$ temperature yielded excessive current over $27 \mathrm{kV}$ (figure 56). Fluctuations in migration time were readily observed. Fluctuations on area counts were also observed. This may be due to the instrument being a brand new $\mathrm{CE}$. A system verification analysis was performed that included a DAD test, in order to show that the new instrument and its software are working properly.

Using the $\mathrm{CE}$ specifications described in the methods section, $0.3 \mathrm{mg} / \mathrm{ml}$ standards of flunitrazepam, diazepam, and alprazolam were observed to migrate at 7.9 minutes, 8.7 
minutes, and 8.3 minutes respectively (figures 57-59). The internal standard, tetracaine, migrated at 6.9 minutes.

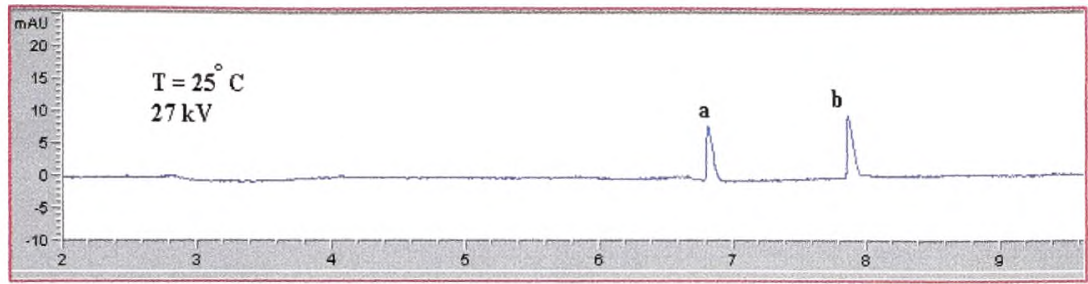

a) tetracaine (ISTD)

b) flunitrazepam

* Garcia and Almirall. AAFS Proceedings $2000{ }^{65,66}$

Figure 57: Flunitrazepam standard at $0.3 \mathrm{mg} / \mathrm{mL}$ and tetracaine standard at $0.2 \mathrm{mg} / \mathrm{mL}$, using $50 \mathrm{mM}$ phosphate $\mathrm{pH} 2.5,210 \mathrm{~nm}$, and a $50 \mathrm{um} \times 48.5 \mathrm{~cm}$ column on a Hewlett Packard 3D CE. ${ }^{65,66}$

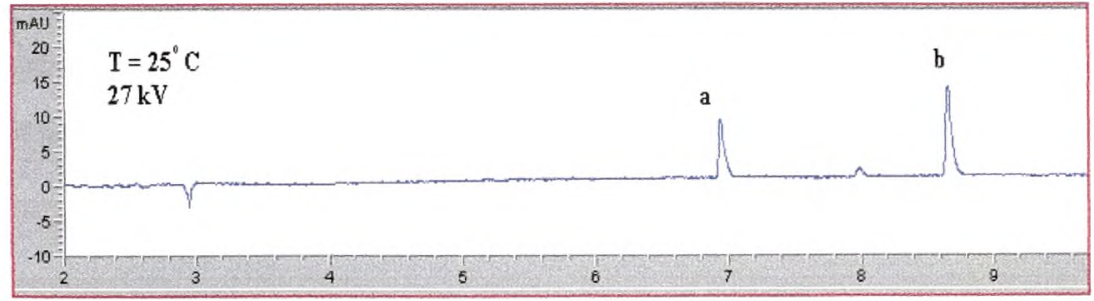

a) tetracaine (ISTD)

b) diazepam

* Garcia and Almirall. AAFS Proceedings $2000 .^{65,66}$

Figure 58: Diazepam standard at $0.3 \mathrm{mg} / \mathrm{mL}$ and tetracaine standard at $0.2 \mathrm{mg} / \mathrm{mL}$, using $50 \mathrm{mM}$ phosphate $\mathrm{pH} 2.5,210 \mathrm{~nm}$, and a $50 \mathrm{um} \times 48.5 \mathrm{~cm}$ column on a Hewlett Packard 3D CE. ${ }^{65,66}$ 


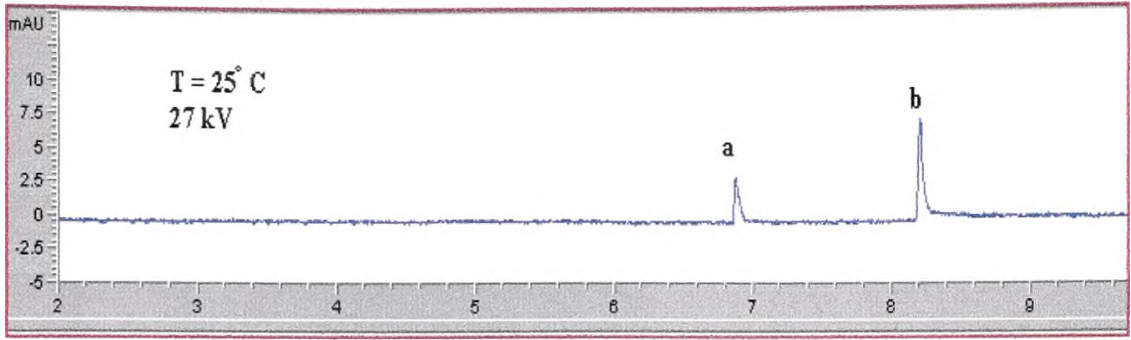

a) tetracaine (ISTD)

b) alprazolam

* Garcia and Almirall. AAFS Proceedings 2000. ${ }^{65,66}$

Figure 59: Alprazolam standard at $0.3 \mathrm{mg} / \mathrm{mL}$ and tetracaine standard at $0.2 \mathrm{mg} / \mathrm{mL}$, using $50 \mathrm{mM}$ phosphate $\mathrm{pH} 2.5,210 \mathrm{~nm}$, and a $50 \mathrm{um} \times 48.5 \mathrm{~cm}$ column on a Hewlett Packard 3D CE. ${ }^{65,66}$

There was a linear response between the concentration and peak areas for diazepam from 0.04 to $0.80 \mathrm{mg} / \mathrm{mL}$ (figure 60 ). For flunitrazepam, the data produced a linear response from 0.10 to $1.1 \mathrm{mg} / \mathrm{mL}$ (figure 61). Migration times for flunitrazepam and diazepam at different concentrations of the standards were reproducible with an RSD of less than 3\% RSD (tables 4 and 5). 


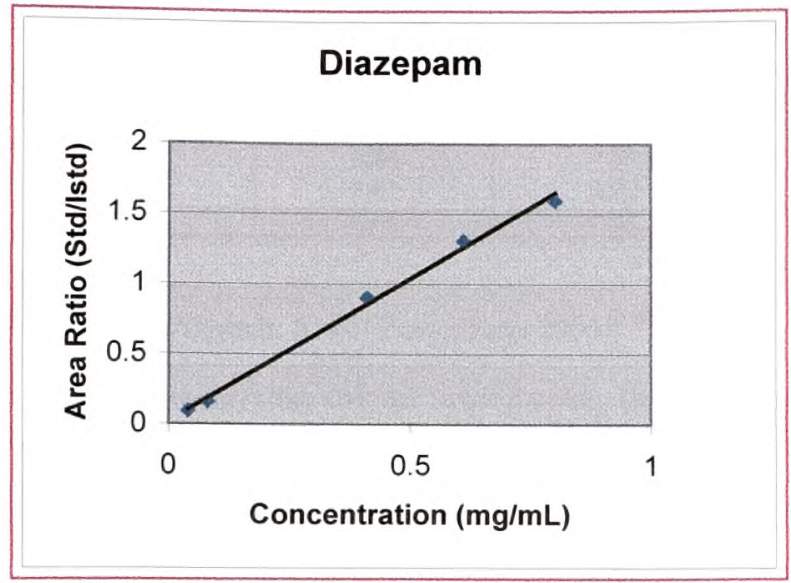

* Garcia and Almirall. AAFS Proceedings 2000. ${ }^{65,66}$

Figure 60: Linearity of diazepam $(0.04$ to $0.80 \mathrm{mg} / \mathrm{ml})$, using $50 \mathrm{mM}$ phosphate $\mathrm{pH} 2.5$, $210 \mathrm{~nm}$, and a $50 \mathrm{um}$ x $48.5 \mathrm{~cm}$ column on a Hewlett Packard 3D CE. ${ }^{65,66}$

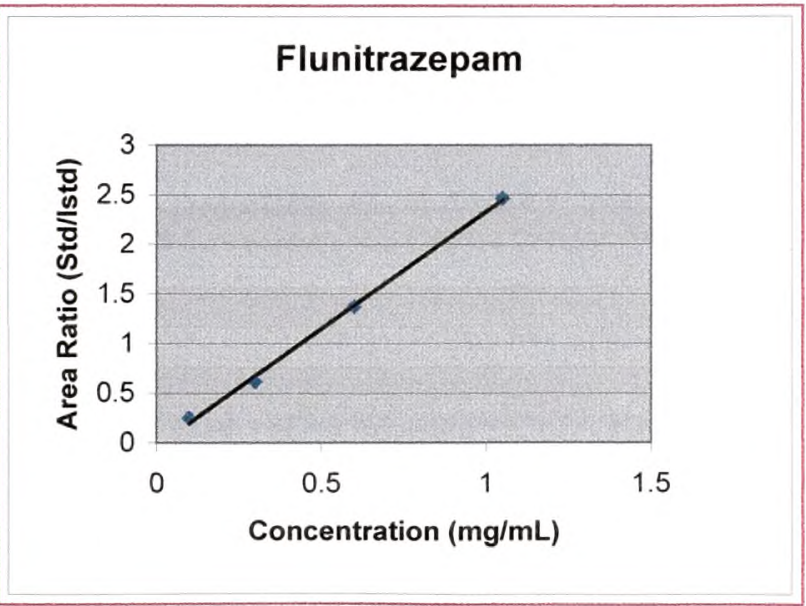

* Garcia and Almirall. AAFS Proceedings 2000. ${ }^{65,66}$

Figure 61: Linearity of flunitrazepam $(0.10$ to $1.1 \mathrm{mg} / \mathrm{ml})$, using $50 \mathrm{mM}$ phosphate $\mathrm{pH}$ $2.5,210 \mathrm{~nm}$, and a $50 \mathrm{um} \times 48.5 \mathrm{~cm}$ column on a Hewlett Packard 3D CE. ${ }^{65,66}$ 


\begin{tabular}{|c|c|c||}
\hline $\begin{array}{c}\text { Concentration } \\
\begin{array}{c}\text { Flunitrazepam } \\
(\mathrm{mg} / \mathrm{mL})\end{array}\end{array}$ & $\begin{array}{c}\text { Flunitrazepam } \\
\text { MT \%RSD } \\
(\mathrm{n}=5)\end{array}$ & $\begin{array}{c}\text { Tetracaine } \\
\text { MT \%RSD } \\
(\mathrm{n}=5)\end{array}$ \\
\hline 1.05 & 1.62 & 1.83 \\
0.60 & 0.44 & 0.88 \\
0.30 & 0.41 & 0.92 \\
0.10 & 0.41 & 0.81 \\
\hline
\end{tabular}

* Garcia and Almirall. AAFS Proceedings 2000. ${ }^{65,66}$

Table 4: Reproducibility data for flunitrazepam, using $50 \mathrm{mM}$ phosphate $\mathrm{pH} 2.5,210 \mathrm{~nm}$, and a $50 \mathrm{um} \times 48.5 \mathrm{~cm}$ column on a Hewlett Packard 3D CE. ${ }^{65,66}$

\begin{tabular}{||c|c|c||}
\hline $\begin{array}{c}\text { Concentration } \\
\text { Diazepam } \\
(\mathrm{mg} / \mathrm{mL})\end{array}$ & $\begin{array}{c}\text { Diazepam } \\
\text { MT \%RSD } \\
(\mathrm{n}=5)\end{array}$ & $\begin{array}{c}\text { Tetracaine } \\
\text { MT \%RSD } \\
(\mathrm{n}=5)\end{array}$ \\
\hline 0.80 & 1.19 & 1.09 \\
0.61 & 2.06 & 1.50 \\
0.41 & 1.31 & 1.17 \\
0.08 & 1.09 & 0.99 \\
0.04 & 0.45 & 0.45 \\
\hline
\end{tabular}

* Garcia and Almirall. AAFS Proceedings 2000. ${ }^{65,66}$

Table 5: Reproducibility data for diazepam, using $50 \mathrm{mM}$ phosphate $\mathrm{pH} 2.5,210 \mathrm{~nm}$, and a 50 um x $48.5 \mathrm{~cm}$ column on a Hewlett Packard 3D CE. ${ }^{65,66}$

A cleanup method was implemented every 5 injections. This method consisted of flushing the capillary column with $0.01 \mathrm{~N} \mathrm{NaOH}$ followed by the running buffer. The cleanup step is important in order to keep a clean baseline. The system tubes were washed for 2 minutes with deionized water and then with the running buffer for 0.5 minutes whenever buffers were changed. The injection solvent used for the analysis of 
diazepam and flunitrazepam was $1.0 \mathrm{~N}$ hydrochloric acid, which yields a $\mathrm{pH}$ of 0.1 . At this extreme $\mathrm{pH}$, diazepam was found to breakdown over time to 5-Chloro-2(methylamino)benzophe-none. The diazepam molecule suffers a loss of $39 \mathrm{amu}$, which corresponds to a loss of one nitrogen, 2 carbon atoms, and 1 hydrogen atom. Diazepam standard $(2 \mathrm{~mL})$ was made basic with $\mathrm{NaOH}$ and extracted with ether. The extract solution was then dried down and re-dissolved in methanol. A GC/MS analysis was performed on this methanol solution. The sample and standard solution should be made fresh, to avoid breakdown of diazepam.

Dilute acid solutions, such as $0.1 \mathrm{~N}$ and $0.01 \mathrm{~N}$ hydrochloric acid, were also attempted as injection solvents for diazepam and flunitrazepam. However, solubility problems were encountered at high concentrations of the drugs. While diazepam is soluble in methanol, flunitrazepam is only slightly soluble. Diazepam is only slightly soluble in water and flunitrazepam is insoluble. Acetic acid (1.0N) and phosphoric acid $(0.1 \mathrm{~N})$ also showed to cause solubility problems for the analysis of flunitrazepam. Phosphate buffer (50mM, pH 2.5)/phosphoric acid (50mM) was also tried as injection solvent for flunitrazepam. However, solubility problems continued at high concentrations of flunitrazepam. Methanol was added to the running buffer $(50 \mathrm{mM}$ phosphate buffer $\mathrm{pH} 2.5$ ), in an attempt to enhance the solubility of diazepam. However, area response was greatly reduced. Future method developments strategies should include the effect of adding surfactants or cyclodextrins to the running buffer. Adding methanol or acetonitrile to the running buffer or just to the injection solvent, to improve the solubilities of diazepam and flunitrazepam, should be explored in greater depth. 
Most of the benzodiazepines were successfully separated in this experiment (figure

62). However, solubility problems were encountered at high concentrations of the drugs.

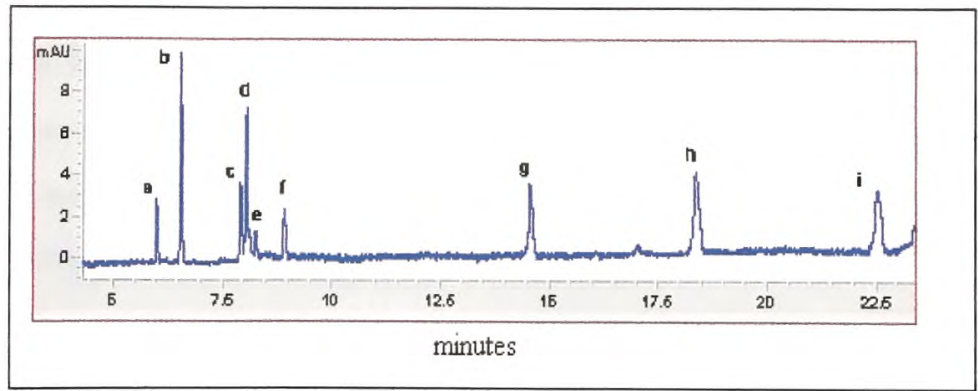
a) clonazepam
f) nitrazepam
b) tetracaine
g) flurazepam
c) flunitrazepam
h) temazepam
d) alprazolam
i) lorazepam
e) diazepam

* Garcia and Almirall. AAFS Proceedings 2000. ${ }^{65,66}$

Figure 62: Common benzodiazepines separated using the CZE method of 50mM phosphate $\mathrm{pH} 2.5,210 \mathrm{~nm}$, and a 50um x $48.5 \mathrm{~cm}$ column on a Hewlett Packard 3D CE. ${ }^{65,66}$ 


\subsection{HPLC RESULTS AND DISCUSSION}

\subsection{GHB}

The Mesmer and Satzger 1998 method resolves both GHB and GBL, using a pH buffer system in the range of $\mathrm{pH} 3 .^{41}$ Although the authors ensure that there is no interconversion of GHB to GBL in this acidic environment during the actual analytical run, leaving a sample or a standard of GHB in an acidic buffer system will interconvert the GHB compound to its lactone GBL through esterification, as demonstrated in the 2001 FDA study of chemical interconversion of GHB and GBL. ${ }^{22}$ Using a pH buffer system that does not favor an acidic or basic environment might demonstrate a non-bias approach to the analysis of GHB/GBL samples in a court of law. Therefore, the current study reports the results at a $\mathrm{pH}(6.5)$ closer to neutral. Several columns were explored during this project and the column with the highest retention for GHB was found to be the C18 Aqua column. Several buffer systems were also explored and due to the high polarity of GHB, a weak aqueous buffer system had the most potential for retaining GHB.

Using the $250 \times 4.6 \mathrm{~mm} \mathrm{C} 18$ Aqua column and a $25 \mathrm{mM}$ Phosphate buffer $(\mathrm{pH}$ 6.5), GHB eluted at 3.3 minutes (figure 63). 


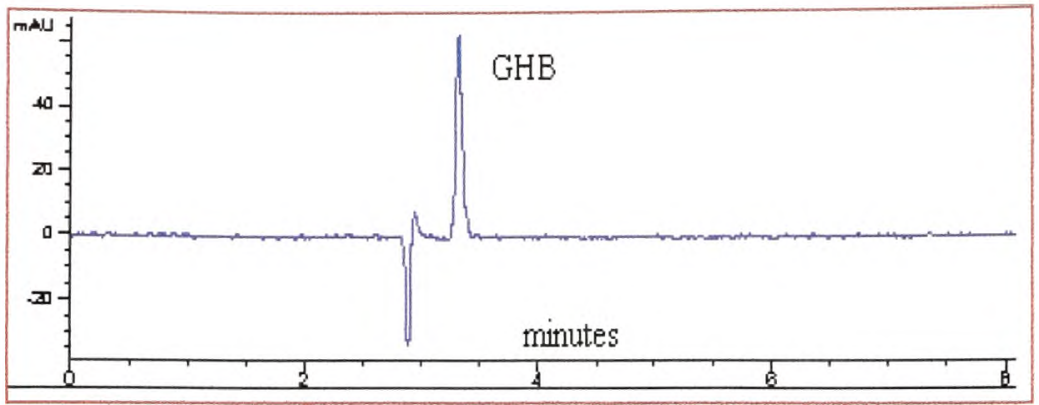

* Garcia, Lurie, Hulett, Almirall AAFS 2001 Proceedings. ${ }^{59,63}$

Figure 63: GHB (3.3 minutes) eluted using a $25 \mathrm{mM}$ phosphate $\mathrm{pH} 6.5,195 \mathrm{~nm}$, and 250 $\mathrm{x} 4.6 \mathrm{~mm}$ C18 Aqua column on a Hewlett Packard 3D CE. ${ }^{59,63}$

GHB yielded a linear response from $0.32 \mathrm{mg} / \mathrm{ml}$ to $5.04 \mathrm{mg} / \mathrm{ml}$ with a correlation coefficient of 0.9998 and a precision below $3 \%$ RSD. The capacity factor $\left(\mathrm{k}^{\prime}\right)$ with this methodology was 0.22 . The capacity factor $\left(\mathrm{k}^{\prime}\right)$ is defined as the ratio of the time the component spends in the stationary phase to the mobile phase. ${ }^{11}$ The formula for calculating $\mathrm{k}^{\prime}$ is as follows:

$$
k^{\prime}=\frac{t_{(r)}-t_{0}}{t_{0}}
$$

where $\mathrm{k}^{\prime}$ is the capacity factor, $\mathrm{t}_{(\mathrm{r})}$ is the retention time for the peak of interest from the point of injection, and $t_{0}$ is the retention time for a non-retained peak. ${ }^{11}$ If the purpose of the analysis was to analyze only GBL, the retention time for GBL can be shortened by 
strengthening the mobile phase through the addition of an organic modifier to the system (figure 64).

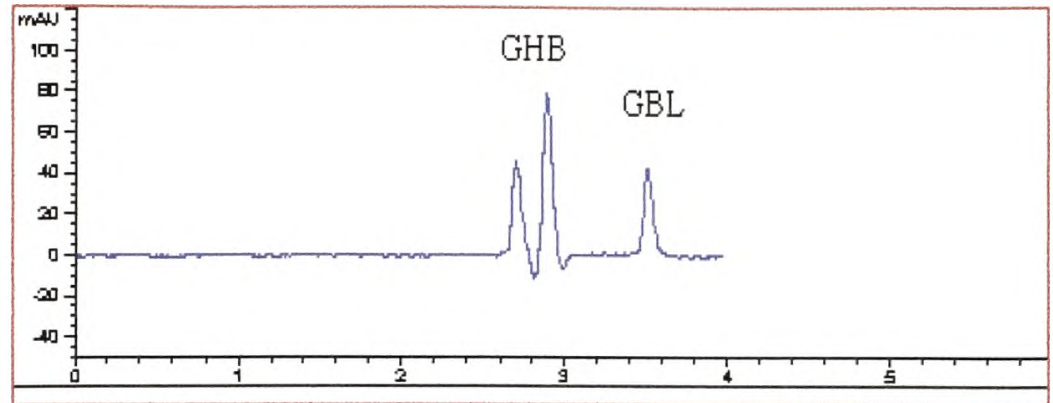

* Garcia, Lurie, Hulett, Almirall AAFS 2001 Proceedings. ${ }^{59,63}$

Figure 64: GHB and GBL using $25 \mathrm{mM}$ phosphate $\mathrm{pH} 6.5 / 10 \%$ methanol, $195 \mathrm{~nm}$, and a $250 \times 4.6 \mathrm{~mm} \mathrm{C18}$ Aqua column on an Agilent HPLC 1100.59,63

GBL gave a linear response from $0.95 \mathrm{mg} / \mathrm{ml}$ to $15.3 \mathrm{mg} / \mathrm{ml}$ with a correlation coefficient of 0.9999 and a precision below 3\% RSD.

Using the shorter C18 Aqua column and 25mM Phosphate buffer ( $\mathrm{pH}$ 6.5), GHB eluted at 2.03 minutes, and GBL eluted at 5.31 minutes (figure 65). GHB gave a linear response from $0.16 \mathrm{mg} / \mathrm{ml}$ to $5.03 \mathrm{mg} / \mathrm{ml}$, and $\mathrm{GBL}$ was linear from $0.65 \mathrm{mg} / \mathrm{ml}$ to 10.30 $\mathrm{mg} / \mathrm{ml}$. The k' was found to be 0.11 . Quantitation results for actual street case samples using both the HPLC and CE methods were comparable (table 6). 


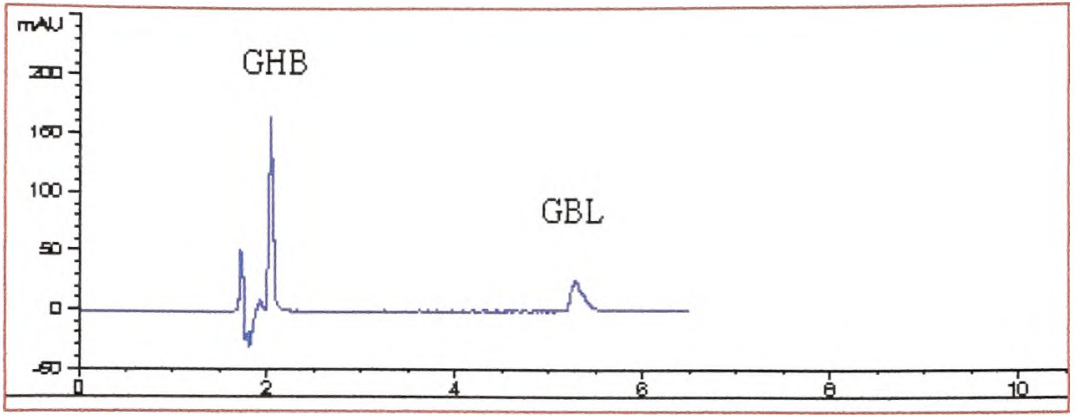

* Garcia, Lurie, Hulett, Almirall AAFS 2001 Proceedings. ${ }^{59,63}$

Figure 65: GHB and GBL using $25 \mathrm{mM}$ phosphate $\mathrm{pH} 6.5,195 \mathrm{~nm}$, and a $150 \times 4.6 \mathrm{~mm}$ C18 Aqua column on an Agilent HPLC $1100 .^{59,63}$

\begin{tabular}{|c|cc|cc||}
\hline \multirow{2}{*}{$\begin{array}{c}\text { Sample } \\
\text { No. }\end{array}$} & \multicolumn{2}{|c|}{ HPLC } & \multicolumn{2}{c||}{ CE } \\
\hline 1 & GHB $(\mathrm{mg} / \mathrm{ml})$ & GBL $(\mathrm{mg} / \mathrm{ml})$ & GHB $(\mathrm{mg} / \mathrm{ml})$ & GBL $(\mathrm{mg} / \mathrm{ml})$ \\
\hline 2 & 42 & 74 & 40 & 76 \\
3 & 40 & 73 & 41 & 75 \\
4 & 38 & 70 & 40 & 72 \\
5 & 54 & 101 & 51 & 98 \\
\hline
\end{tabular}

* Garcia, Lurie, Hulett, Almirall AAFS 2001 Proceedings. ${ }^{59,63}$

Table 6: Comparison of actual GHB cases using $25 \mathrm{mM}$ phosphate $\mathrm{pH} 6.5$ and a $250 \mathrm{x}$ $4.6 \mathrm{~mm} \mathrm{C18}$ Aqua column on an Agilent HPLC 1100, compared to $25 \mathrm{mM}$ phosphate/300mM CTAC pH 6.3 on a Hewlett Packard 3D CE. ${ }^{59,63}$ 
GHB and GBL standards at different concentration were analyzed for reproducibility. There was excellent reproducibility with the longer $\mathrm{C} 18$ column (table 7), yielding $\%$ RSD of less than $3 \%$.

\begin{tabular}{|c|c|c|c|}
\hline Compound & Avg. Migration & \%RSD & R \\
& Time & $(\mathrm{n}=5)$ & Resolution \\
\hline GHB & 2.032 & 0.054 & $*$ \\
\hline GBL & 5.217 & 0.013 & 3 \\
\hline
\end{tabular}

* Garcia, Lurie, Hulett, Almirall AAFS 2001 Proceedings. ${ }^{59,63}$

Table 7: Reproducibility of GHB and GBL standards using $25 \mathrm{mM}$ phosphate $\mathrm{pH} 6.5$ and a $250 \times 4.6 \mathrm{~mm}$ C18 Aqua column on an Agilent HPLC $1100 .^{59,63}$

The case samples obtained for the comparison between HPLC and CE were dietary supplements and commercial solvents containing GHB and GBL sold for human consumption. Analysis of these types of samples is more difficult than typical street samples of GHB and GBL. Their sample matrix and much lower concentrations of GHB and GBL make analytical procedures more challenging. Low concentrations of GHB are more difficult to detect (in the presence of GBL), using techniques such as nuclear magnetic resonance (NMR) or infrared spectroscopy (IR) due to GBL masking any presence of GHB. Thus, the presence of only GBL existing in the sample could falsely be reported. 
The LC method used in this study did not incorporate an organic solvent. Since GHB is a very polar compound, a typical C18 column does not retain the compound sufficiently for effective separations. Furthermore, a purely aqueous mobile phase would deteriorate most C18 column, by causing the octadecyl chains to collapse. ${ }^{68}$ The Aqua C18 column is "endcapped" with a hydrophilic reagent. ${ }^{68}$ This "polar endcapping" allows polar compounds to be retained (dipole-dipole interaction of analyte with stationary phase) and gives reproducible separations using a purely aqueous mobile phase. ${ }^{68}$

Although a low capacity factor ( $\left.\mathrm{k}^{\prime}\right)$ was obtained for GHB using both LC columns, no interferences have been observed in over 50 actual case samples of GHB in different matrices. Since a potential exists for possible interferences, the scientist can check the purity of the UV spectra observed, if necessary. The capacity factor can be increased by addition of a salt to the buffer system or a decrease in temperature. ${ }^{11}$

\subsection{Phenylethylamines}

Five case samples of methamphetamine cases were examined and results were compared to GC and CE (table 8). Five samples of MDMA tablets were also examined by HPLC, and results were compared to CE and GC values (table 9). For both methamphetamine and MDMA samples, there was comparable results between GC, HPLC, and CE analysis. 


\begin{tabular}{|c|c|c|c|}
\hline $\begin{array}{c}\text { sample } \\
\text { No. }\end{array}$ & $\begin{array}{c}\text { GC } \\
\%\end{array}$ & $\begin{array}{c}\text { HPLC } \\
\%\end{array}$ & $\begin{array}{c}\text { CE } \\
\%\end{array}$ \\
\hline 1 & 32.7 & 32.3 & 31.2 \\
2 & 6.1 & 5.9 & 5.5 \\
3 & 2.3 & 2.1 & 2.0 \\
4 & 1.9 & 1.6 & 1.6 \\
5 & 66.6 & 68.2 & 67.3 \\
\hline
\end{tabular}

* Garcia and Lurie. IAFS 1999 Proceedings. ${ }^{60}$

Table 8: Comparison of 5 actual street methamphetamine samples using GC, HPLC, and CE. ${ }^{60}$

\begin{tabular}{|c|c|c|c|}
\hline $\begin{array}{c}\text { Sample } \\
\text { No. }\end{array}$ & $\begin{array}{c}\text { GC } \\
\text { mg/tablet }\end{array}$ & $\begin{array}{c}\text { HPLC } \\
\text { mg/tablet }\end{array}$ & $\begin{array}{c}\text { CE } \\
\text { mg/tablet }\end{array}$ \\
\hline 1 & 91 & 91 & 88 \\
2 & 111 & 110 & 108 \\
3 & 106 & 109 & 108 \\
4 & 107 & 104 & 105 \\
5 & 83 & 84 & 80 \\
\hline
\end{tabular}

* Garcia and Lurie. AAFS 2000 Proceedings. ${ }^{6}$

Table 9: Comparison of 5 actual street MDMA samples using GC, HPLC, and CE. ${ }^{61}$ 


\subsection{Psilocybin}

Good agreement was obtained for the analysis of psilocybin samples analyzed by CE and HPLC, as seen in table 10. Using, methanol and acetonitrile, $100 \mathrm{ppm}$ of psilocybin standard was ran as a reference on an Agilent HPLC 1100, for the quantitation of the different mushrooms samples (figure 66). The only mushroom found to contain traces of psilocin was mushroom 4, which was verified by both HPLC (figure 60) and CE analysis.

\begin{tabular}{||c|c|c|c|c|}
\hline Mushroom & mass & \multicolumn{3}{|c|}{ \% Psilocybin } \\
\cline { 3 - 5 } No. & mg & CZE & MECC & HPLC \\
\hline 1 & 592.1 & 0.86 & 0.82 & 0.92 \\
2 & 242.8 & 0.98 & 0.94 & 1.04 \\
3 & 1486.8 & 0.16 & 0.16 & 0.16 \\
4 & 615.1 & 0.20 & 0.28 & 0.30 \\
5 & 2018.5 & 0.38 & 0.38 & - \\
\hline
\end{tabular}

* Garcia, Gross and Almirall. IAFS 1999 Proceedings. ${ }^{62}$

Table 10: Psilocybin values compared by HPLC, MECC, and CZE. ${ }^{62}$ 


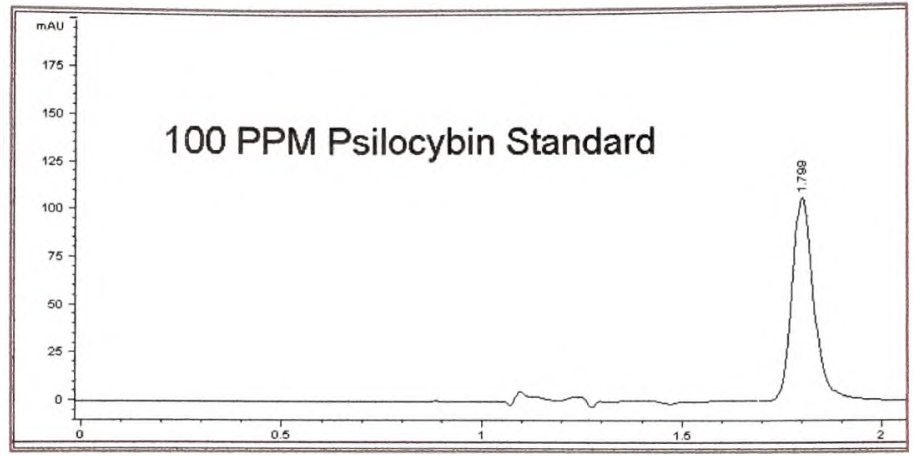

* Garcia, Gross and Almirall. IAFS 1999 Proceedings. ${ }^{62}$

Figure 66: Psilocybin standard using an acetonitrile/methanol buffer system on an Agilent HPLC $1100 .^{62}$

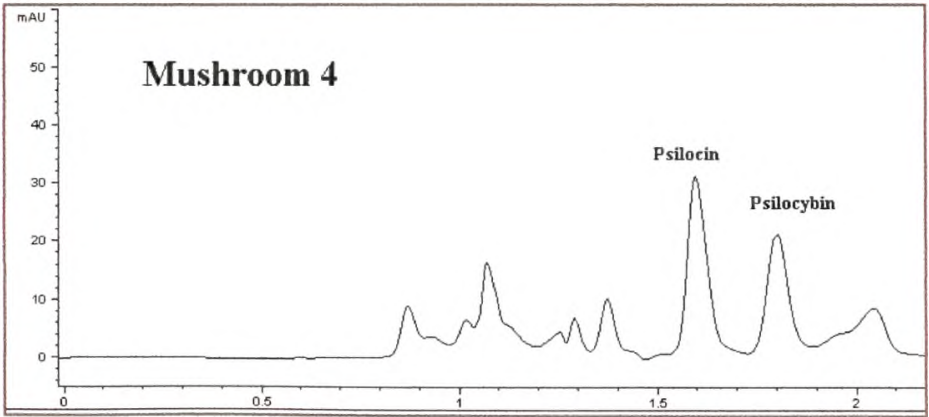

* Garcia, Gross and Almirall. IAFS 1999 Proceedings. ${ }^{62}$

Figure 67: Mushroom 4 using an acetonitrile/methanol buffer system on an Agilent HPLC $1100 .^{62}$ 


\subsection{Opium}

Good agreement between the analysis of opium via CE dynamic coating and HPLC was found. CE analysis for an opium sample is shown in figure 68, while opium analysis via HPLC is shown in figure 69. CE separation of opium alkaloids were greater than those achieved via HPLC. Both methods were used at multiple wavelengths. At $210 \mathrm{~nm}$ morphine, codeine, thebaine, and noscapine had increased sensitivity. Since HPLC can detect all classes of solute and CE can only detect moderate and strongly basic compounds, a larger number of peaks were seen via HPLC analysis. ${ }^{4}$ 


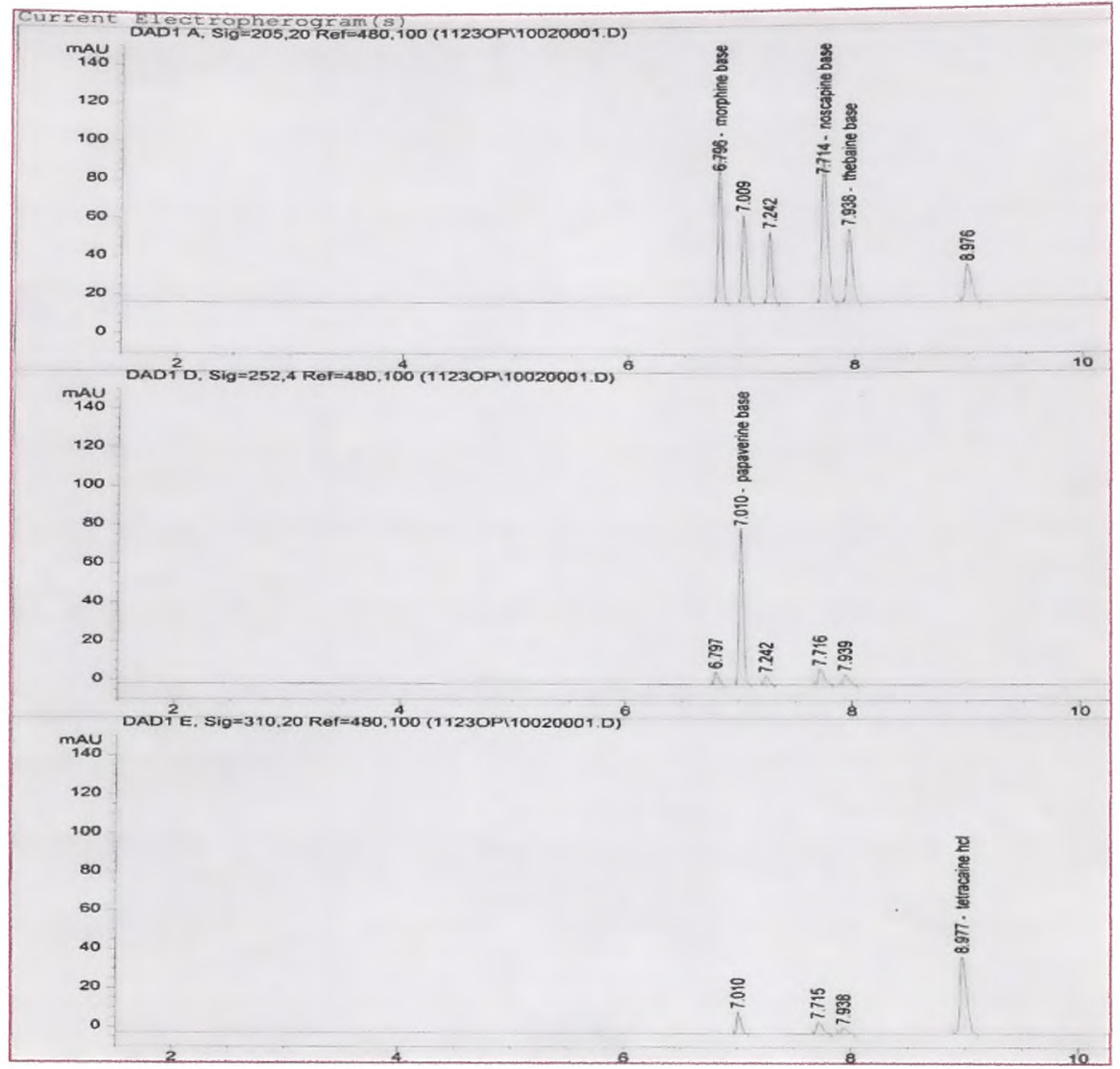

Mixture of morphine (6.7 $\mathrm{min})$, papaverine $(7.0 \mathrm{~min})$, codeine $(7.3 \mathrm{~min})$, noscapine (7.7 min), thebaine $(7.9 \mathrm{~min})$, and tetracaine $(8.9 \mathrm{~min})$ as the internal standard.

Figure 68: Dynamic Coating for Opium, using 50mM of DM-B-CD/CElixir pH 2.5. 


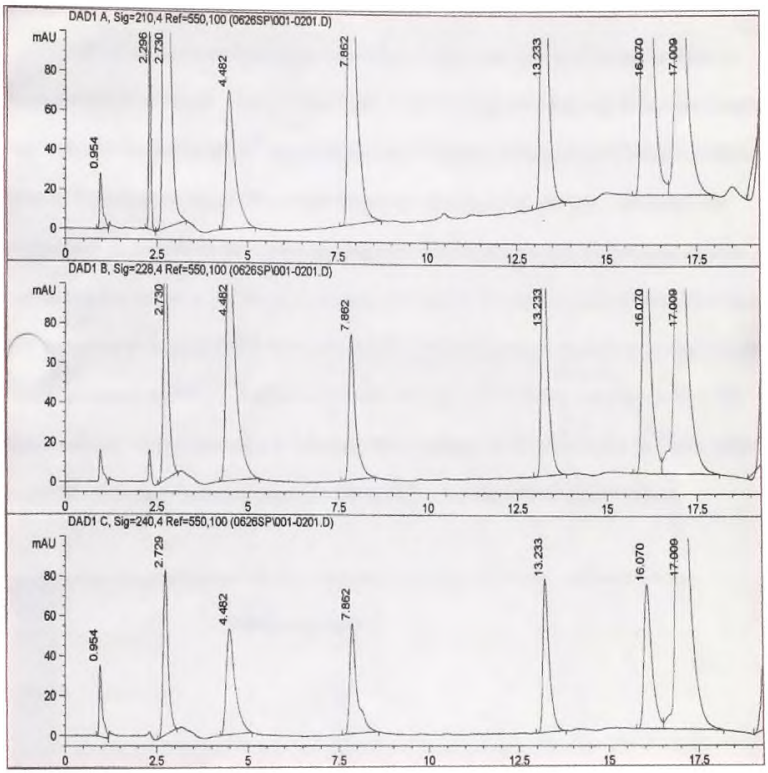

Figure 69: Opium analysis via HPLC method. 


\subsection{Benzodiazepines}

HPLC analysis of flunitrazepam and diazepam was also performed in order to compare to CE analysis. Using a 5 um ODS $150 \times 3.2 \mathrm{~mm}$ column, a $210 \mathrm{~nm}$ wavelength, and a phosphate buffer $\mathrm{pH}$ 2.3/acetonitrile buffer system, flunitrazepam had a retention time of 1.9 minutes (figure 70), while diazepam eluted at 2.2 minutes. Although the differences in migration time were not large, the differences in UV absorbance spectra can utilized to confirm the identity of each compound. Flunitrazepam absorbed the most UV radiation at around 200-210nm (figure 72), while diazepam peaked at around $240 \mathrm{~nm}$. Since diazepam and flunitrazepam are most often seen in forensic laboratories in tablet form, solvent washes are used to separate the diazepam or flunitrazepam from the tablet material. Common solvents used are chloroform, methanol, and ether washes.

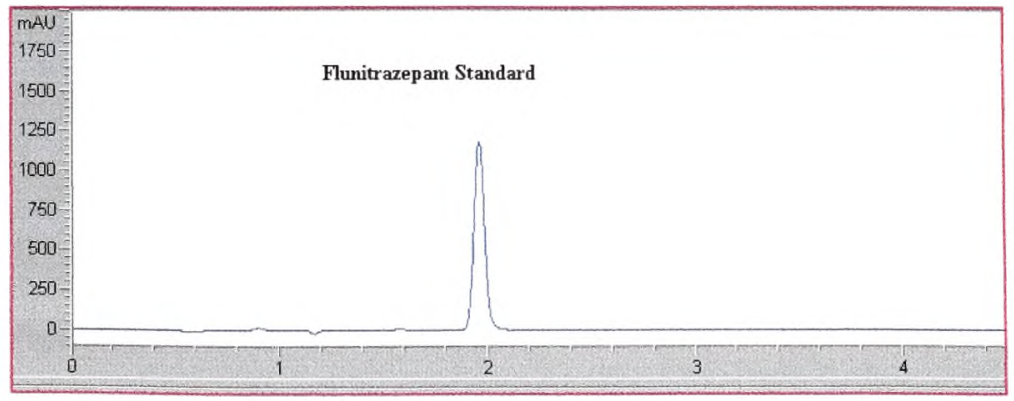

Figure 70: Flunitrazepam standard $(1.0 \mathrm{mg} / \mathrm{mL})$ using a 5 um ODS $150 \times 3.2 \mathrm{~mm}$ column, $210 \mathrm{~nm}$, and a 50/50 phosphate $\mathrm{pH}$ 2.3/acetonitrile buffer system on an Agilent HPLC 1100 . 


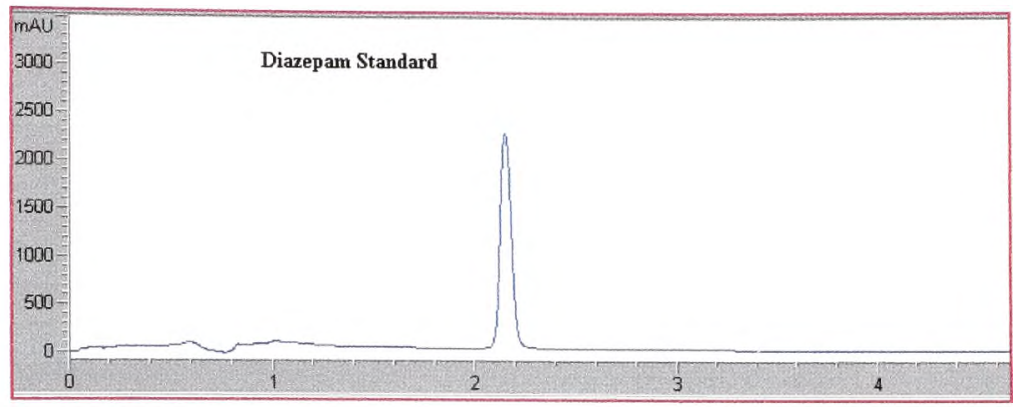

Figure 71: Diazepam standard $(1.0 \mathrm{mg} / \mathrm{mL})$ using a 5 um ODS $150 \times 3.2 \mathrm{~mm}$ column, 210 $\mathrm{nm}$, and a 50/50 phosphate $\mathrm{pH}$ 2.3/acetonitrile buffer system on an Agilent HPLC 1100.

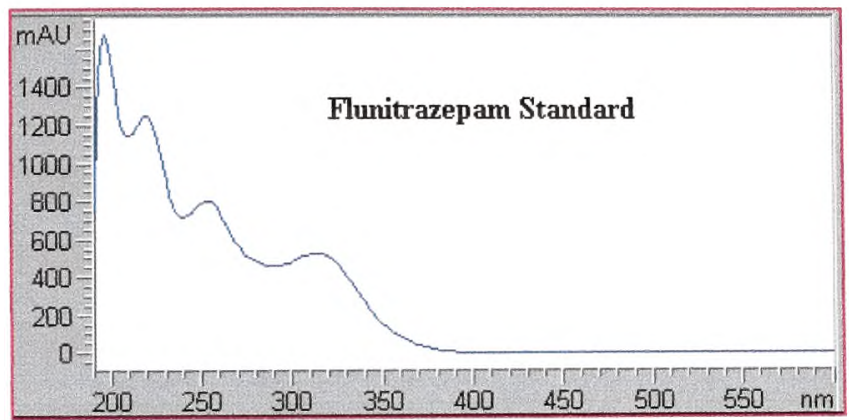

Figure 72: UV spectra of flunitrazepam. 


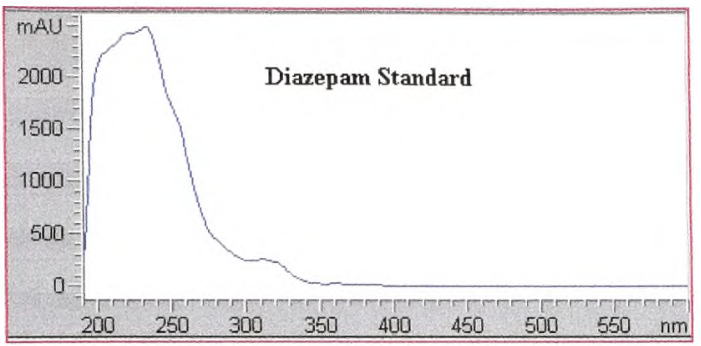

Figure 73: UV spectra of diazepam. 


\subsection{CONCLUSIONS}

Both CE and HPLC are valid techniques for screening and quantitation of GHB/GBL samples. A reverse phased HPLC system incorporating a C18 Aqua column and a 25mM Phosphate buffer ( $\mathrm{pH} 6.5$ ) resulted in migration times of 3.3 minutes and 8.9 minutes for GHB and GBL, respectively. GHB was found to yield a linear response from $0.32 \mathrm{mg} / \mathrm{ml}$ to $5.04 \mathrm{mg} / \mathrm{ml}$ with a correlation coefficient of 0.9998 and a precision below $3 \%$ RSD. A capillary electrophoresis separation employing 50mM CTAC and $25 \mathrm{mM}$ phosphate ( $\mathrm{pH} 6.4$ ) resulted in a migration time of $1.86 \mathrm{~min}$. and 2.82 minutes for GHB and GBL, respectively. GHB gave a linear response from $0.039 \mathrm{mg} / \mathrm{ml}$ to $10.01 \mathrm{mg} / \mathrm{ml}$ with correlation coefficient 0.9999 a precision below $5 \%$ RSD, using a system incorporating 300mM CTAC.

Capillary electrophoresis allows for the analysis on nonvolatile, polar, and thermally degradable compounds. This study has shown that CZE and MECC are fast and reliable analytical methods for the determination and quantitation of psilocybin in illicit mushrooms.

Dynamic coating is a fast and rugged technique for the analysis of phenylethylamines and opium alkaloids, as shown by this study. The analysis of benzodiazepines using a $50 \mathrm{mM}$ phosphate $\mathrm{pH} 2.5$ running buffer was found to separate 9 benzodiazepines. However, diazepam broke down to 5-chloro-2(methylamino)benzophenone in the injection solvent of $1.0 \mathrm{~N}$ hydrochloric acid.

In conclusion, this study has demonstrated the advantages of using CE versus HPLC and GC. For thermally degradable compounds like GHB and psilocybin, GC techniques are not suitable without derivatization. While HPLC techniques can analyze 
thermally degradable compounds, CE offers much higher efficiency (theoretical plates in the hundreds of thousands as oppose to thousands with HPLC). This study also proved how CE can offer higher resolution in the analysis of optical isomers, without the need for derivatization. Although chiral columns are available for GC and HPLC, there are no methods for the simultaneous separation of all 18 phenylethylamines with these techniques. In many instances, a forensic laboratory will receive a sample that contains several of the phenylethylamines in one sample matrix, such as tablets with methamphetamine, amphetamine, MDMA, and MDEA. The forensic chemist would benefit from a method that would be suitable for both optical isomers determination and quantitation. In addition, the high resolution and efficiency that CE offers over HPLC in the profiling of opium samples cannot be compared to any other technique. Opium alkaloid content (even in very small concentrations) is very important in the profiling of opium samples.

Not only does capillary electrophoresis offer more analytical options to the forensic chemist, but generates little solvent waste (due to little solvent consumption), utilizes inexpensive columns (approximately $\$ 5$ a column), and employs little use of toxic organic solvents. 


\section{LIST OF REFERENCES}

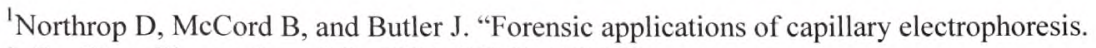
J. Capillary Electrophoresis", 1994; 001(2):158-168.

${ }^{2}$ Lurie I. "Analysis of seized drugs by capillary electrophoresis", In: J. A. Adamovies, editor. Analysis of Addictive and Misused Drugs, Marcel Decker: New York. 1994; 151219.

${ }^{3}$ Heiger D. High Performance Capillary Electrophoresis. Agilent Technologies. 2000.

${ }^{4}$ Weinberger R and Lurie I. "Micellar electrokinetic capillary chromatography of illicit drug substances", Anal. Chem. 1991; 63:823-827.

${ }^{5}$ Chankvetadze, B. Capillary Electrophoresis in Chiral Analysis, John Wiley \& Sons Ltd., 1997.

${ }^{6}$ Terabe, S., "Micellar Electrokinetic Chromatography", Beckman Primer.

${ }^{7}$ Landers, J. Handbook of Capillary Electrophoresis. Second Edition. CRC Press. 1997.

${ }^{8}$ Chevigne and Janssens, US patent $\# 5,611,909,3 / 18 / 97$.

${ }^{9}$ CElixir. Scientific Resources, Inc.

${ }^{10}$ Graul TW, Schlenoff JB. Capillaries modified by polyelectrolyte multilayers for electrophoretic separations. Anal chem. 1999; 71:40007-13.

${ }^{11}$ Snyder L, Kirkland J, and Glajch J. Practical HPLC Method Development. John Wiley \& Sons, Inc., second edition. 1997.

${ }^{12}$ Uwe N. HPLC Columns. Wiley-VCH, Inc. 1997.

${ }^{13}$ Lurie, American Laboratory. 1980.

${ }^{14}$ Walker JA, Krueger ST, Lurie IS, Marche HL, and Newby NN. Analysis of Heroin Drug Seizures by Micellar Electrokinetic Capillary Chromatography (MECC). J Forensic Sciences. 1995; 40(1): 6-9.

${ }^{15}$ Lurie IS. American Laboratory. 1996; 28:26-34.

${ }^{16}$ Lurie IS, Sperling AR, and Meyers RP. The Determination of Anabolic Steroids by MECC, Gradient HPLC, and Capillary GC. J Forensic Sciences. 1994; 39(1): 74-85. 
${ }^{17}$ Lurie IS, Dal Cason TA, LeBelle MJ, Brenneisen R, and Weinberger RE. "Chiral Resolution of Cationic Drugs of Forensic Interest by Capillary Electrophoresis with Mixtures of Neutral and Anionic Cyclodextrins", Analytical Chemistry. 1994; 66(22): 4019-4026.

${ }^{18}$ Tagliaro F, Manetto G, Bellini S, Scarcella D, Smith F, and Marigo M. "Simultaneous chiral separation of 3,4-methylenedioxymethamphetamine (MDMA), 3-4methylenedioxyamphetamine (MDA, 3,4-methylenedioxyethylamphetamine (MDE), ephedrine, amphetamine, and methamphetamine by capillary electrophoresis in uncoated and coated capillaries with native B-cyclodextrin as the chiral selector: Preliminary application to the analysis of urine and hair", Wiley-VCH, 1998.

${ }^{19}$ Lurie IS, Bethea MJ, McKibben TD, Hays PA, Pellegrini P, Sahai R., Garcia AD, and Weinberger R. "Use of Dynamic Coated Capillaries for the Routine Analysis of Methamphetamine, Amphetamine, MDA, MDMA, MDEA, and Cocaine Using Capillary Electrophoresis", J Forensic Sciences. 2001. 46(5): 1025-1032.

${ }^{20}$ Lurie IS. Personal Communication.

${ }^{21}$ Iwanicki RM, Maier K, Zlotnick JA, Liu RH, Kuo TL, and Tagliaro F. "Separation of Enantiomeric Ephedrine and Pseudoephedrine - High Pressure Liquid Chromatography and Capillary Electrophoresis", J Forensic Sciences. 1999; 44(3):470-474.

${ }^{22}$ Ciolino L, Mesmer M, Satzger R, Machal A, McCauley H, Mohrhaus A. "The chemical interconversion of GHB and GBL: forensic issues and implications", $\mathrm{J}$ Forensic Sci 2001;46(6):1315-1323.

${ }^{23}$ LeBeau M A, Montgomery M A, Jufer R A, Miller M L. "Elevated in citrate-buffered blood", J Analytical Tox 2000; 24:383-384.

${ }^{24}$ Meyers J E, Almirall J R. "Analysis of gamma-hydroxybutyric acid (GHB) in spiked water and beverage samples using solid phase microextraction (SPME) on fiberderivatization/gas chromatography-mass spectrometry (GC/MS)", J Forensic Sci.

Submitted 2003.

${ }^{25}$ Garcia and Almirall. "Fast Analysis of Gamma-Hydroxybutyric Acid (GHB) by In-Situ Derivatization on an SPME Fiber", Proceedings of the American Academy of Forensic Sciences 1999.

${ }^{26}$ United States Sentencing Commission Guidelines Manual. 1988; 101

${ }^{27}$ Lurie I et al. "Capillary electrophoresis of illicit drug seizures", Forensic Science International. 1988; 92:125-136. 
${ }^{28}$ Decision Of Appeal On Controlled Substances Analogue Act. J CLIC. 2004; 14(1):1314

${ }^{29}$ Lewis A and Smith E. "Gamma-hydroxybutyrate: From the 60 s to the present", Proceedings of the American Academy of Forensic Sciences 2000; 6:43.

${ }^{30}$ Duchaine D. "Underground steroid handbook (II). Update: 1992", Golden, CO: Mile High Publishing, 1992.

${ }^{31}$ Phillips WN. "Anabolic reference guide", $6^{\text {th }}$ ed. Golden, CO: Mile High Publishing. 1991.

${ }^{32}$ Frommhold S. "Gamma-hydroxybutyrate (GHB): What's "the Scoop"?", Houston Police Department Crime Laboratory, Houston, Texas.

${ }^{33}$ Fact Sheet: GHB. U. S. Department of Justice, Drug Enforcement Administration, Public Affairs Section. October. 1996.

${ }^{34}$ Sannerud CA. "Gamma Hydroxybutyrate (GHB)", DEA Office of Diversion Control. December 1998 .

${ }^{35}$ ElSohly M and Salamone S. "Prevalence of drugs used in cases of alleged sexual assault", Journal of Analytical Toxicology; 23:141-146.

${ }^{36}$ Elian A. "A novel method for GHB detection in urine and its application in drug facilitated sexual assaults", Forensic Science International. 2000;109(3):183.

${ }^{37}$ Karch, S., "GHB abuse and detection", Forensic Drug Abuse Advisor, Vol. 12, No. 1, 2000, pp. 1-2.

${ }^{38}$ Drug Enforcement Administration, "Gamma hydroxybutyric acid", Washington, D.C.: Press Release March 2000.

${ }^{39}$ Sannerud CA. Personal Communication. DEA Office of Diversion Control.

${ }^{40}$ Federal Register: November 25, 2003. 68(227).

${ }^{41}$ Mesmer, M. and Satzger, R. "Determination of gama-hydroxybutyrate (GHB) and gamma-butyrolactone (GBL) by HPLC/UV-VIS spectrophotometry and HPLC/thermospray mass spectrometry", Journal of Forensic Sciences 1998;43(3):489492.

${ }^{42}$ Meyers, J.E. and Almirall, J.R. "A Study of the Interconversion of Gammahydroxybutyric Acid (GHB) and Gamma-Butyrolactone (GBL) in Solution at Various pH's Over Time", Journal of Forensic Sciences, Submitted 2003. 
${ }^{43}$ Krawezeniuk, A., "Direct Detection of Gamma Hydroxybutyrate: A New Recreational Drug via Free-Zone Capillary Electrophoresis", Proceedings of the American Academy of Forensic Sciences, p. 34.

${ }^{44}$ Blackledge, R. and Miller, M. "Gammahydroxybutyrate GHB Somatomax PM IR GCMS BSTFA Gamma-Butyrolactone" Microgram, Vol. 24, No. 7, July 1991, pp. 172-179.

${ }^{45}$ Bommarito, C. "Analytical Profile of Gamma-HydroxyButyric Acid (GHB)", Journal of the Clandestine Laboratory Investigating Chemists Association, Vol. 3, No. 3, July 1993, pp. 10-12.

${ }^{46}$ McKibbens T. Personal Communication. DEA Special Testing and Research Laboratory.

${ }^{47}$ Catterton A. ATR Analysis of GHB. Journal of the Clandestine Laboratory Investigating Chemists Association, 2000.

${ }^{48}$ Chew S. Quantitation of GHB by NMR. Microgram.

${ }^{49}$ Morris JA. Analogs of GHB. Part 2: Analytical Perspective. J CLIC. 2000; 10(2): 1820.

${ }^{50}$ Morris JA. Analogs of GHB. Part 2: Analytical Perspective. J CLIC. 2001; 11(1): 1630 .

${ }^{51}$ Karch SB. Drug Abuse Handbook. CRC 1998.

${ }^{52}$ Garcia A. and Catterton A. "1,4-Butanediol - A Forensic Profile", Microgram 2002

${ }^{53}$ Inaba, D., Cohen, W., and Holstein, M. "Upper, Downers, All Arounders", Third Edition, Second Printing, CNS Publications, Inc., 1997.

${ }^{54}$ Drug Identification Bible 2003, Amera-Chem Inc., 2003.

${ }^{55}$ Thomson BM. "Analysis of Psilocybin and Psilocin in Mushroom Extracts by Reversed-Phase High Performance Liquid Chromatography", J Forensic Sciences. 1980; 25(4):779-785.

${ }^{56}$ Yuko, Garcia, Kanamori, Inoue, Kishi, and Lurie. "The Use of a Highly sulfated cyclodextrin for the simultaneous chiral separation of amphetamine-type stimulants by capillary electrophoresis", Electrophoresis 2002, 23, 1328-1334.

${ }^{57}$ Hudson JC, Golin M, Malcolm M, and Whiting CF. "Capillary Zone Electrophoresis in a Comprehensive Screen for Drugs of Forensic Interest in Whole Blood: An Update", Can. Soc. Forens. Sci. J. 1998; 31(1): 1-29. 
${ }^{58}$ Lurie IS, Panicker S, Hays PA, Garcia AD, and Geer BL. "Use of dynamically coated capillaries with added cyclodextrins for the analysis of opium using capillary electrophoresis", J. of Chromatography A, 984 (2003), 109-120.

${ }^{59}$ Garcia A., Lurie I., Hulett, L. and Almirall J., "Quanitation of GHB and GBL using Capillary Electrophoresis and HPLC", Proceedings of the American Academy of Forensic Sciences 2001.

${ }^{60}$ Garcia A. and Lurie I., "Simultaneous Chiral Determination and Quantitation of Methamphetamine and Related Compounds Using Capillary Electrophoresis", International Association of Forensic Sciences Conference 1999.

${ }^{61}$ Garcia A. and Lurie I., "Quantitation of 3.4-Methylenedioxymethamphetamine and Related Compounds using Capillary Electrophoresis", American Academy of Forensic Sciences Proceedings 2000.

${ }^{62}$ Garcia A., Gross S., and Almirall J., "Quantitative Analysis of Psilocybin and Psilocin from the Psilocybe Cyanescens (Wakefield) Mushroom by CE", International Association of Forensic Sciences Conference 1999.

${ }^{63}$ Garcia A., Hulett L., Lurie I., and Almirall J., "Quantitation of GHB and GBL using Capillary Electrophoresis and HPLC", International Association of Forensic Sciences Conference 1999.

${ }^{64}$ Bjornsdottir SH. and Hansen J., J Pharm. Biomed. Anal. 13 (1995) 687.

${ }^{65}$ Garcia A., "Analysis of Flunitrazepam and Diazepam Using Free Zone Capillary Electrophoresis", Southern Association of Forensic Scientists Conference 1998.

${ }^{66}$ Garcia A. and Almirall J., "Quantitative Analysis of Common Benzodiazepines by Free Zone Electrophoresis", American Academy of Forensic Sciences Proceedings 2000.

${ }^{67}$ Garcia A., Lurie I., and Almirall J., "A Comprehensive Review of the Analysis of Controlled Substances by Capillary Electrophoresis, Using Free Zone, MECC, and Cyclodextrin Systems", American Academy of Forensic Sciences Proceedings 2000.

${ }^{68}$ Phenomenex. "Aqua" a new C18 column with polar end-capping. Torrance, CA.

${ }^{69}$ Gorman C. Liquid X A club drug called GHB may be a fatal aphrodisiac. Time. 1996; 148(16):64.

${ }^{70}$ Marwick C. Coma-inducing Drug GHB may be reclassified. JAMA 1997; 277(19):1505-1506. 
${ }^{71}$ McCauley H. et al. GHB, GBL, BD: A Recipe for Disaster. Journal of Forensic Sciences, in press.

${ }^{72}$ Ropero-Miller, J.D. and Fraser, D. B. "GHB and Roofies: Drugs of the 1990s" Forensic Urine Drug Testing: An AACC Educational Newsletter for Laboratories, June 1997.

${ }^{73}$ Salvatore, S., Aturki, Z., and Desidero, C., "New strategies for chiral analysis of drugs by capillary electrophoresis", Forensic Science International, Vol. 92, 1998, pp. 137-155.

${ }^{74}$ Takahara J, Yunoki S, Yakushiji W, Yamauchi J, Yamane Y, and Ofuji T. Stimulatory effects of Gamma-Hydroxybutyric acid on growth hormone and prolactin release in human. J. Clin. Endocrinol. Metab. 1977;44:1014-16.

${ }^{75}$ Walker, J., Marché, H., Newby, N., and Bechtold, E., "A free zone capillary electrophoresis method for the quantitation of common illicit drug samples", $\mathbf{J}$ Forensic Sci, Vol. 41, No. 5, 1996, pp. 824-829. 